\title{
Nanoparticles for Radiooncology: Mission, Vision, Challenges
}

Leoni A. Kunz-Schughart, ${ }^{1}$ Anna Dubrovska, ${ }^{1}$ Claudia Peitzsch, ${ }^{1}$ Alexander Ewe, ${ }^{2}$ Achim Aigner, ${ }^{2}$ Samuel Schellenburg, ${ }^{3}$ Michael H. Muders, ${ }^{3}$ Silke Hampel, ${ }^{4}$ Giuseppe Cirillo, ${ }^{5}$ Francesca Iemma, ${ }^{5}$ Rainer Tietze, ${ }^{6}$ Christoph Alexiou, ${ }^{6}$ Holger Stephan, ${ }^{7}$ Kristof Zarschler, ${ }^{7}$ Orazio Vittorio, ${ }^{8}$ Maria Kavallaris, ${ }^{8}$ Wolfgang J. Parak, ${ }^{9,10}$ Lutz Mädler, ${ }^{11}$ Suman Pokhrel ${ }^{11^{*}}$

${ }^{1}$ OncoRay-National Center for Radiation Research in Oncology, Faculty of Medicine and University Hospital Carl Gustav Carus, Technische Universität Dresden and Helmholtz-Zentrum Dresden-Rossendorf, Dresden, Germany; ${ }^{2}$ Rudolf-Boehm-Institute for Pharmacology and Toxicology, Clinical Pharmacology, University of Leipzig, Germany; ${ }^{3}$ Institute of Pathology, University Hospital, Carl Gustav Carus, TU Dresden, Germany; ${ }^{4}$ Leibniz Institute of Solid State and Material Research Dresden, 01171 Dresden, Germany; ${ }^{5}$ Department of Pharmacy, Health and Nutritional Sciences, University of Calabria, 87036 - Rende (CS) Italy; ${ }^{6}$ ENT-Department, Else Kröner-Fresenius-Stiftung Professorship, Section for Experimental Oncology and Nanomedicine (SEON), University Hospital Erlangen, Erlangen, Germany; ${ }^{7}$ Helmholtz-Zentrum DresdenRossendorf, Institute of Radiopharmaceutical Cancer Research, 01314 Dresden, Germany; ${ }^{8}$ Children's Cancer Institute Australia, ARC Centre of Excellence in Convergent Bio-Nano Science and Technology and Australian Centre for NanoMedicine, Sydney, UNSW, Australia; ${ }^{9}$ Fachbereich Physik, Philipps Universität Marburg, 35037 Marburg, Germany; ${ }^{10} \mathrm{CIC}$ Biomagune, 20009 San Sebastian, Spain; ${ }^{11}$ Foundation Institute of Materials Science (IWT), Department of Production Engineering, University of Bremen, 28359 Bremen, Germany

\section{Corresponding author}

Dr. Suman Pokhrel

Institute of Materials Science (IWT)

Department of Production Engineering, University of Bremen, 28359 Bremen,

Bremen, Germany

Phone: +49-421-21851218

Email: spokhrel@iwt.uni-bremen.de 


\begin{abstract}
Cancer is one of the leading non-communicable diseases with highest mortality rates worldwide. About half of all cancer patients receive radiation treatment in the course of their disease. However, treatment outcome and curative potential of radiotherapy is often impeded by genetically and/or environmentally driven mechanisms of tumor radio-resistance and normal tissue radio-toxicity. While nanomedicine-based tools for imaging, dosimetry and treatment are potential keys to the improvement of therapeutic efficacy and reducing side effects, radiotherapy is an established technique to eradicate the tumor cells. In order to progress the introduction of nanoparticles in radiooncology, due to the highly interdisciplinary nature, expertise in chemistry, radiobiology and translational research is needed. In this report recent insights and promising policies to design nanotechnology-based therapeutics for tumor radio-sensitization will be discussed. An attempt is made to cover the entire field from preclinical development to clinical studies. Hence, this report illustrates (1) radio- and tumor biological rationale for combining nanostructures with radiotherapy, (2) tumor-site targeting strategies and mechanisms of cellular uptake, (3) biological response hypotheses for new nanomaterials of interest, and (4) challenges to translate the research findings into clinical trials.
\end{abstract}

\title{
Contents
}

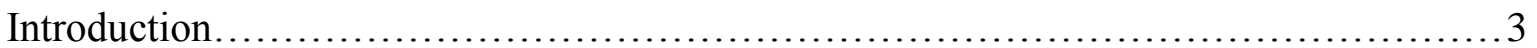

Cellular irradiation effects and tumor biological rationales.......................... 5

Combination treatment for improvement of radiotherapy efficacy $\ldots \ldots \ldots \ldots \ldots \ldots \ldots \ldots 6$

Nanoparticle delivery of siRNA for therapeutic applications ........................ 10

Structure meets function: carbon nanostructure-based drug delivery $\ldots \ldots \ldots \ldots \ldots \ldots \ldots \ldots . \ldots \ldots$

Polymer functionalization for therapeutics ...................................... 17

Tumor targeting with nanomaterials............................................22

Promising yet challenging performance: magnetic delivery strategies.................. 24

Renal clearable nanomaterials with tumor specific binding as putative radio-sensitizers... 27

Hypothesis-driven development of new NPs for cancer therapy..................... 29

Demanding intermediate: preclinical models for nanoparticle evaluation............... 31

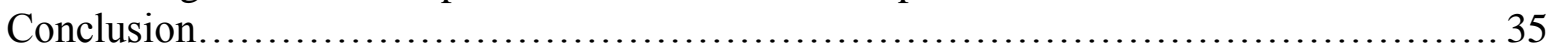

Acknowledgements...................................................... 35

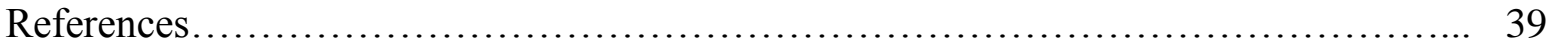




\section{Introduction}

According to the world health organization (WHO), the number of cancer-related mortalities per year is projected to increase by $45 \%$ from 2007 to 2030, influenced in part by an increasing global aging population. In today's society, the costs of cancer care are enormous, where the EU spends annually $\sim 126$ billion $€$. More than 14 million new cases and $>8$ million cancer deaths were reported worldwide during 2012 to 2013, with an elevating trend described in GLOBOCAN by the International Agency for Research on Cancer (IARC) and the Institute for Health Metrics and Evaluation (IHME) [1, 2]. These data underline the urgent need for a re-evaluation and prioritization of new approaches to complement and improve current diagnostic tools and treatment methods. The latter comprises the three main pillars of cancer treatment, namely surgery, radio and chemotherapy, which were over the past decade extended by a range of novel chemotherapeutic as well as individually applicable targeted therapeutics and immunotherapies. Patients with specific malignant diseases clearly benefit from the reasonable progress in surgical and chemotherapeutic treatment. However, only marginal improvement in overall clinical management of cancers patients could be achieved, with some malignant diseases such as pancreatic cancers and glioblastomas, as well as most advanced stage cancers, remaining an unsolved therapeutic challenge. Here, the most prominent limitations of currently available treatment options such as dose-limiting toxicity, lack of specificity, selectivity, bioavailability of drug candidates or local distribution, and morbidity become particularly apparent. Novel strategies that are generally applicable, have high (local) efficacy and are cost-efficient, and of utmost urgency [3]. A great hope lies in the field of nanomedicine, where nanoparticles (NPs) can be specifically designed using advanced engineering tools to treat and visualize tumors. Several nanoparticles (NP)-based formulations are undergoing clinical trials, or are even already used in clinics [4, 5]. Most applications however merely utilize NPs as drug delivery vehicles for or as mediators in physical anticancer methods, such as heating of tumor cells. In particular the delivery vehicle aspect has been critically discussed recently [6]. These methods suffer from several drawbacks, such as the need for advanced NP surface chemistry, specialized equipment, lack of specificity, low efficiency in drug release rates, and undesired NP toxicity [7-11]. For imaging applications, NPs either contain intrinsic contrast (e.g. $\mathrm{FeO}_{\mathrm{x}}$ cores for magnetic resonance imaging $(\mathrm{MRI}))$, or are further functionalized through chemical means (e.g. 
fluorescence or radiolabeled probes) [12]. These functionalities enable follow-up of the NPs' location after administration, but do not give any direct information on the ongoing therapy.

Photodynamic therapy (PDT) consists of light, photosensitizer and oxygen during treatment. The mechanism of the photodynamic involves activating photosensitizers via certain wavelength of light followed by emission (recombination). The whole excitation-emission process is accompanied with the energy release that is transferred to the near surface oxygen generating ROS (singlet oxygen, free radicals and/or superoxide) [13]. The chemical reactions take place during PDT is (1) the direct interaction of excited photosensitizers with the cell membrane or the cellular components transferring $\mathrm{H}$ atom to form potentially hazardous radicals (2) direct energy transfer from the excited photosensitizers to surface oxygen generating singlet oxygen $\left({ }^{1} \mathrm{O}_{2}\right)$ and/or highly oxidizing superoxide [13]. Hence, for the progress in the cancer therapy especially phototherapeutic technology, the development of new light sensitive photosensitizers is necessary. During treatment, these efficient photosensitizers are expected to be cleared from the body faster and absorb light at higher wavelengths leading to a limited period of photosensitivity in the targeted area [14-19]. Designing such sensitizers (altered or mixed to target specific cell abnormalities) targeting various organs and parts of the cell such as membrane and lysosomes are very promising in treatment of tumors. Although Photodynamic therapy is one of the effective techniques to treat cancer, it has a serious drawback. These effective photosensitizers with reduced duration of light irradiation have intense and prolonged chemical reactions post treatment [20]. Hence, in the future, the photodynamic therapy (PDT) in combination with the surgery and radiotheraphy could be uniquely tailored to treat cancers [21]. The treatment includes the development of new photosentizers, using optimal photodynamic therapy protocols (light fraction and/or drug dose) [22, 23]. Furthermore, the clinical trials involving selective and friendly sensitizers with low energetic light irradiation may improve the photodynamic therapy technique in cancer treatment $[24,25]$.

The use of NPs in the context of radiotherapy is a particular issue that has been challenging in the past. Radiotherapy as one of the key modalities to treat solid cancers is the major treatment option beyond surgery with high curative potential. Today, about 50-60\% of the cancer patients receive radiotherapy, most frequently in entity-specific combinatorial radio/chemotherapeutic approaches [26, 27]. The success rate and outcome of patients is still limited by normal tissue toxicities and the development of individual, highly variable intrinsic as well as 
microenvironmentally-driven tumor therapy resistances that require improvement and optimization of the current treatment policies [27-31]. Here development of novel strategies and types of nanoparticles and -materials, in particular to ameliorate the cancer-specific efficacy of radiotherapy would be highly helpful. It is recognized that some materials might be considered as dosimetric in -vivo nanosensors to monitor therapeutic levels of ionizing radiation as recently shown for C12 TAB-templated gold NPs exhibiting unique spectral profiles under ionizing radiation [3]. However, in this report focus will be given rather describing a vision of NPs for radio-sensitization based on the cellular irradiation effects and tumor biological rationales, as depicted in the following. Therapeutic challenges will be highlighted and some specific examples of interest are given.

\section{Cellular irradiation effects and tumor biological rationales}

Radiotherapy may eradicate cancer cells through a set of physical and chemical changes induced in the tumor tissues via transmitted energy. Many different types of ionizing radiation have been employed for medical diagnostic and therapeutic applications including photons (X-rays, gamma rays), leptons (electrons), hadrons (negative pi-mesons, neutrons, protons) and heavier ions (carbon, silicon, neon, helium). The major considerations for selections of the certain type of ionizing radiation for medical use include its controllability within an atomic site, inherent pattern of ionizing density defined by the linear energy transfer (LET), and relative biological effectiveness (RBE), attributed to the relative biological effects per unit energy [32]. Up to date, X-rays (photons) remain the most common type of radiation therapy due to its low production cost [33]. State-of-the-art photon radiotherapy is based on continuous technological progress over the past decades that led to an advanced 3D conformal treatment, and includes the use of intensity-modulated radiation therapy (IMRT) techniques with in-room image guidance (imageguided radiation therapy). Particle therapy with protons or heavier ions, such as carbons have the potential for higher dose conformity compared with photon beams, due to a reverse depth dose profile, i.e. particle beams can be directed more precisely as they deposit most of their energy over a narrow range (Bragg peak) [34-39]. The energy of the beam defines the depth of the Bragg peak in tissue and can be modulated to achieve maximum ionization within the tumor site and spare organs of risk to minimize normal tissue injury. Although high equipment and facility costs are the major obstacle for wider applications, proton and carbon ion therapy has been shown to be 
an efficient treatment modalities for different types of malignancies, including head and neck squamous cell carcinoma (HNSCC), prostate, brain, and pediatric cancers [40-42]. More details on the technical improvements in photon and particle therapy have been discussed in a recent report highlighting the efforts in biology-driven precision radiation oncology [27]. Despite improved precision of radiotherapy delivery, treatment-related toxicities often show late effects. The intrinsic and environmentally-driven tumor radio-resistance, tumor metastasis, poor diseasefree and overall survival of cancer patients, remains a clinical and scientific challenge [29, 31].

The curative potential of irradiation mainly depends on its ability to induce non-repairable DNA damage in tumor cells, either by direct ionization of the DNA molecules, or by generation of free radicals, including oxygen-derived chemically reactive products $[26,43]$. Tolerance to DNA damage-induced cell death via activation of pro-survival signaling cascades (e.g. phosphatidylinositol-3 kinases (PI3K/AKT), nuclear factor $\kappa \mathrm{B}(\mathrm{NF}-\kappa \mathrm{B})$, and mitogen-activated protein kinase (MAPK)) are distinctive feature of cancer cells that might reduce the efficacy of radiotherapy (Figure 1) [28]. Beside the intrinsic mechanisms affecting tumor response to radiation, micro-environmental constraints such, as the intra-tumoral oxygen level, also play an important role for tumor radio-curability. The oxygen distribution in solid malignant tissues is inhomogenous, due to a pathological capillary network in the growing tumor mass which is unorganized, leaky, fragile, and shows perfusion malfunctions. This goes along with non-(patho)physiological and steep spatiotemporal and micro-regional oxygen gradients resulting in chronic, diffusion-limited as well as acute, perfusion-limited, and intermittent oxygen deficiencies (hypoxia) [44, 45]. Cancer cells residing in hypoxic areas can be more shielded from radiationinduced DNA damage due to reduced ROS generation and activation of pro-survival signaling pathways, e.g. via the regulation of hypoxia-inducible factor (HIF)/HIF-1 $\alpha$-dependent transcriptional control $[28,46]$.

\section{Combination treatment for improvement of radiotherapy efficacy}

The results of preclinical and clinical studies (combination of radiotherapy and chemotherapy) hinted that the judicial selection of the drug combinations might enhance tumor sensitivity to radiotherapy, thus allowing lower total irradiation doses and/or shorter exposure times [47]. One option is to combine radiotherapy with cytotoxic and/or target-specific drugs. Here, selection of the most promising agents for combination is critical to guarantee a reasonable therapeutic 
window and to avoid severe side effects, as shown recently for example for some, but not all inhibitors of epidermal growth factor receptor (EGFR) [48]. Today, it is well known, that enhanced radio-response might in principle be achieved by various drug treatment strategies. These treatments include (1) chemicals that mimic the radio-sensitizing effect of oxygen by metabolic interference, e.g. by impacting oxidative phosphorylation and/or reducing local oxygen deficiencies (acting via different mechanisms as functional oxygen mimetics), (2) inhibitors directed against DNA damage response (DDR) molecules and DNA binding molecules such as specific PARP (Poly-ADP-ribose polymerase) or HDAC (histone deacetylases) family members, as well as (3) antibodies and inhibitors targeting receptor tyrosine kinases (RTK) such as EGFR and/or the respective signaling pathways $[49,50]$. Accordingly, a broad range of clinical studies to combine radiotherapy with novel drugs and targeted therapies has been initiated over the past decade, as exemplified and functionally classified in Table 1.

Table 1. Selected tumor radio-sensitizer currently used in clinical trials (selected).

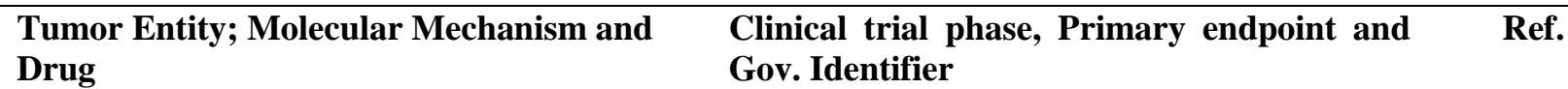

Inhibitor Class 1 (DDR Inhibitor)

Laryngeal, rectal, breast cancer, HNSCC; $\quad$ Phase I: Dose limiting toxicities

PARP inhibitor; Drug: Olaparib NCT02229656, NCT01589419,

$[51,52]$

NCT01477489

Solid tumors refractory to conventional

Phase I: Maximum tolerated dose NCT02223923

AZD6738

SCLC, rectal cancer; Topoisomerase inhibitor;

Drug: Topoteca

Phase I, II: Safety, efficacy

NCT00043862, NCT00158886, NCT00215956

Inhibitor Class 2 (Kinase/RTK inhibitor)

CRC, HNSCC; EGFR targeting antibody Drug: Cetuximab

CRC, EGFR targeting antibody;

Drug: Panitumumab

Esophageal Squamous Cell Carcinoma, EGFR targeting antibody; Drug: Nimotuzumab
Phase III: FDA approved; Overall survival NCT00673738, NCT00815308 NCT00343083, NCT00124618

Phase II: FDA approved, Overall survival, $\quad[61,62]$ loco-regional control NCT00798655, NCT00547157

Phase II/III: Disease free survival, Overall survival 
Pancreatic, rectal, esophageal cancer, metastatic NSCLC, GBM, HNSCC, EGFR small molecule inhibitor; Drug: Erlotinib

Soft tissue sarcoma, prostate cancer, VEGF inhibitor; Drug: SU5416

Prostate cancer, glioblastoma, HNSCC, Multitargeted RTK (e.g. PDGF, VEGF, c-Kit, FLT, CSF, RET); Drug: Sunitinib

HNSCC, NSCLC, prostate cancer, GBM mTOR inhibitor; Drug: Everolimus

Pancreatic cancer, Malignant Glioma, SCC, Rectal cancer, VEGF inhibitor Drug: Bevacizumab

Liver, prostate cancer, Multi-targeted RTK (e.g. Raf, VEGFR); Drug: Sorafenib

GBM

Src/Abl kinase inhibitor

Drug: Dasatinib

Lung, rectal, pancreas cancer, GBM, HIV protease inhibitor, PI3K/AKT inhibitor;

Drug: Nelfinavir

GMB, NSCLC

PKC inhibitor; Drug: Enzastaurin
NCT02272699, NCT01232374

Phase I/II: Toxicity, Progression-free

survival, Overall survival

[66-69]

NCT00096265, NCT00766636

NCT00410826

Phase I, II/III: Safety and tolerability, Side

$[70-72]$

effects

NCT00023738, NCT00026377

Phase I, II: Maximum tolerated dose, safety and tolerability objective response rate

NCT00631527, NCT01100177

NCT00437372

Phase I, Phase I/II: Toxicity

NCT01217177, NCT00943956

$[78-81]$

NCT00943956

Phase II: Toxicity, safety, efficacy, local tumor response NCT00305877, NCT00782756, NCT00408694 NCT00113230

Phase I/II, III: Safety and tolerability, overall survival

NCT01730937, NCT00924807

Phase II: Overall survival NCT02661113

Phase I, II: Dose Escalation NCT01447589

[90-93]

Phase I: Dose-limiting toxicity NCT00509821, NCT00415363

Inhibitor Class 3 (Functional Oxygen Mimetics)

HNSCC, Fixation of free radicals

Drug: Nimorazole

GBM, Increased Cerebral Oxygen Tension; Drug: NVX-108

High grade Glioma; Oxygen delivery

Drug: Trans sodium crocetinate

HNSCC, Dual CAIX Inhibitor

Drug: DTP348

Recurrent breast Cancer, Hydrogen peroxide $(0.5 \%)$, Induction of oxidative stress;
Phase II: Locoregional control, nodal control, disease free survival, metastasis

[97-99] NCT01880359 NCT01507467

Phase I: Safety and tolerability NCT02189109

Phase I: Safety, and Pharmacokinetic NCT00826930

$[101,102]$

Phase I: Dose-escalation study NCT02216669

[103-105]

Phase I, II: Intratumoral pain, tumor response
[106] 
Inhibitor Class 4 (Other mechanism)

NSCLC, Cox2-inhibitor; Drug: Celecoxib

Brain tumors, HDAC inhibitor;

Drug: Panobinostat

Brain tumors, HDAC inhibitor;

Drug: Valproic acid

Pancreatic cancer, GBM Inhibitor of thioredoxin reductase and ribonucleotide reductase, ROS production

Drug: Motexafin, Gadolinium

SCLC, GBM

Anti-autophagy; Drug: Chloroquine

Prostate cancer; Androgen-deprivation

Drug: Goserelin

Pancreatic cancer, HNSCC

PD-1/PDL-1 inhibitor, immune checkpoint inhibition;

Drug:Pembrolizumab/Tremelimumab

Melanoma, NSCLC, Cervical cancer Anti-cytotoxic T lymphocyte antigen (CTLA4); Drug: Ipilimumab

HNSCC, NSCLC, Rectal cancer

Proteasome inhibitor; Drug: Bortezomib
Phase II: Tumor response rate NCT00181532

$[107,108]$

Phase I: Maximum tolerated dose NCT01324635

$[109,110]$

Phase II: Safety, efficacy, median progression free survival NCT00302159

Phase I: Dose escalation and pharmacokinetic, toxicity, maximumtolerated dose NCT00003411, NCT00032097, NCT00006452, NCT00004262

Phase I: Safety Study NCT01575782

Phase III: Improved 5-year survival rate NCT00423475

Phase I, II: Safety, tolerability $[119,120]$ NCT02311361, NCT02587455 NCT02775812, NCT02735239

Phase II: Response rates

NCT01689974, NCT01557114

NCT01711515, NCT02221739

$[121,122]$

Phase I: Dose-limiting toxicity, maximum tolerated dose; NCT00629226, NCT00093756, NCT01445405,

[123-126] NCT00280176

Pre-clinical (with promising results): - Chk1/2 inhibitor; - DNA-PK inhibitor

Some of the radio-sensitizers work as synthetically lethal combination when administered with irradiation, such as modulators of the DNA damage response [28, 102, 127]. However, despite enormous efforts, many new drugs may (as in the past) fail to improve patient survival due to pharmacokinetic limitations, undesired side effects, and biological toxicities. Furthermore, the increasing knowledge and insights in many radio-sensitizing mechanisms derived from knockdown experiments cannot yet be sufficiently translated into medical approaches because of lack of functional inhibitors. Therapeutic application of small inhibitory RNAs, which are not useful as such in vivo, due to restricted life-time and distribution characteristics, is therefore of great 
interest. The emergence of nanotechnology especially for medical applications offers an avenue towards the use of such novel classes of therapeutics based on small RNA molecules, underlining the rational for NPs in the delivery of radio-sensitizing moieties.

\section{Nanoparticle delivery of siRNA for therapeutic applications}

In the last 15-20 years, the role of certain small RNA molecules in modulating/inhibiting the expression of their target genes has been well established. More specifically, the mechanism of RNA interference (RNAi) in the nematode worm Caenorhabditis elegans was reported in 1998 to specifically silence genes by exogenous double-stranded RNA. Soon after, RNAi was shown to be a highly conserved mechanism in most eukaryotic cells that can be triggered by small interfering RNA [128]. These siRNAs, 21-23 base pairs in length and containing 2-3 nucleotides 3' overhangs, interact with the multifunctional enzyme Argonaute-2 (Ago 2) and are incorporated into the RNA-induced silencing complex (RISC). The activated RNA-induced silencing complex with the intact siRNA guide strand (RISC*) can bind to its target mRNA sequence, and finally cleaves the mRNA into smaller fragments, which are subsequently degraded due to their unprotected terminations. An outstanding property of the RNA interference, in contrast to classic antisense technologies, is the catalytic activity of the activated RISC, while antisense oligonucleotides $(\mathrm{AON})$ inhibit the translational processing by complementary target mRNA binding in a stoichiometric ratio. The silencing effect can last for up to 7 days in fast growing cells, and many weeks in weakly dividing cells [129]. Since all other components of the RNAi machinery are provided by the cell, only the delivery of the siRNA is necessary and sufficient for the knock down of a given gene. In the process, siRNA will be responsible and determine the target gene specificity to inhibit any genes of interest. While in vitro (tissue culture) RNAi is well-established as a tool e.g. in functional (onco-)gene analyses (see below and Fig. 2C, here exemplified by the knockdown of the reporter gene EGFP), the in vivo application leads to novel therapeutic approaches when targeting oncogenes which are rate-limiting for tumor growth (see below and Fig. 2D).

In the context of radio-sensitization, siRNAs have been extensively explored in tissue culture. RNAi screening using pools of different siRNA strands were exploited to identify key components of DNA repair, after using ionizing radiation therapy [130]. During the RNAi screening process, some of the genes associated in DNA repair such as BRCA1 and BRCA2 
and/or POLQ (expressed differentially in cancer tissue), could be detected for their involvement in radio-resistance $[131,132]$. Therapeutically targeting BRCA2 via RNA interference was one of the first successful approaches to radio-sensitize cancer cells in vitro [61]. In another study, the effectiveness of siRNA in radio-sensitization targeted to Ku80 protein (part of the DNA-PK complex) was shown [133]. Very recent studies also demonstrated the importance of RNAi in the radio-sensitization of head and neck squamous carcinoma by using a lentiviral siRNA approach, [134] and Mcl1 protein targeted by siRNA to radio-sensitize pancreatic ductal adenocarcinoma [2]. Additionally, it was clear that Neuropilin-2 and VEGF-C were observed to be potential radiosensitizers $[135,136]$. The impact of the Neuropilin-interacting protein GIPC1 was tested by siRNA preclinically [137]. Non-coding RNAs could be targets of short interfering RNAs [137]. Number of studies has shown the role of long non-coding RNAs (lncRNAs) in radio-resistance of cancer. Wang et al. demonstrated the role of different lncRNAs in the radio-resistance of nasopharyngeal carcinoma [138]. In contrast, there are significantly more studies investigating the role of microRNAs (miRNAs) in radio-resistance. It has already been shown that the DNA repair machinery is also regulated by miRNAs, and that targeting miRNAs could be a viable therapeutic approach [78]. Further studies have confirmed the role of different other miRNAs in radio-resistance [73, 139]. While RNAi offers broad applications for treating undruggable diseases or to specifically target pathologically relevant (overexpressed) genes, a major hurdle is still the delivery of siRNAs into the particular tissue. The large polyanionic molecules are not actively internalized by the cells and are not able to freely cross the cell membrane. Moreover, rapid degradation by serum nucleases and renal clearance further impede organ delivery and cellular uptake. Once taken up, the nucleic acids are prone to the endosomal/lysosomal degradation processes. Furthermore, siRNA molecules can induce an innate immune response in size- and sequence-specific manner by activating toll-like receptors. For addressing these problems, various strategies have been explored. Chemical modifications of the ribose backbone, the introduction of novel nucleotide modifications, and the rational design of the chosen siRNA sequence, have been widely investigated to enhance stability, specificity, improve silencing and mitigate immune reactions [140-142]. In light of concerns associated with the use of viral vectors, various non-viral strategies have been investigated for siRNA delivery [see Figure 2 (A), Figure 3]. These include siRNA conjugation to entities such as lipids, cell penetrating peptides, proteins/antibodies and polymers, acting as self-delivery devices [143-145] $N$ acetylgalactosamine (GalNAc)-siRNAs have already entered several clinical trials. [146] 
However, appropriate systems are only available for certain target organs, with liver hepatocytes being the best example. Alternatively, NP systems made from diverse natural or synthetic materials including polymers, lipids and inorganic materials have been explored [147, 148]. Inorganic nanoparticles (metal, silica) can adsorb siRNAs on their surface or incorporate them in pores (see [149] for review). Liposomes are self-assembled, usually phospholipid-based vesicles that separate an inner aqueous core from surrounding aqueous compartment, and are thus highly attractive delivery systems for a wide range of applications. The amphiphilic character of phospholipids allows for the encapsulation of small lipophilic and hydrophilic drugs as well as large biomolecules in the inner core, in order to improve the drug stability, bioavailability and to minimize side effects [150, 151]. Particularly efficient for nucleic acid delivery are synthetic cationic lipids, which are able to spontaneously form positively charged lipoplexes in the presence of nucleic acids like siRNAs. In the recent years, many synthetic lipids with improved head groups and linker moieties have been introduced and are commercially available for in vitro applications $[152,153]$.

While major issues for their therapeutic application include instability (aggregation, premature release), rapid clearance, immune stimulation (interferon response, inflammation reactions), and probably genotoxicity (as shown for DOTAP) [144, 154-157], these are mainly caused by the cationic charge and can be minimized by the incorporation of polyethylene glycol (PEG)-lipids $[158,159]$. These "stealth liposomes" avoid detection by the immune system due to PEG (polyethylene glycol) covering the outer surface, and also allow for prolonged circulation half lives. "Solid-lipid nanoparticles" are used for siRNA delivery (see [160] for review) and SNALPs (stable nucleic acid lipid nanoparticles) are especially designed for the delivery of siRNAs, combining the key features of classical cationic lipids and conventional liposomes. In several pre-clinical and first clinical studies, these neutrally charged NPs have shown to be well tolerated with significant target gene silencing [138, 161, 162]. Polymeric micelles are selfassembling nano-constructs of amphiphilic block copolymers that form nanoscopic core/shell structures which are used for various applications including gene delivery. On the other hand, cationic polymers are able to electrostatically interact with siRNAs forming nanoparticles [See Figure 2 (B), (C)]. These polymeric nanoparticles can be chemically modified for example with PEG for reduced surface charge and/or with ligands for specific ligand-mediated binding to and uptake into target cells [See Figure 2 (B)]. The combination of polymeric, e.g., PEI-based nanoparticles with liposomes is possible as well. These lipopolyplexes have been explored for 
siRNA delivery in vitro [see Figure 2 (C)] and in vivo [see Figure 2 (D)]. The interaction of siRNAs with several classes of polymers including poly-L-lysine (PLL), polyamidoamine dendrimers (PAMAM), polypropylenimine (PPI) dendrimers and polyethylenimine (PEI) have been studied. In addition, the interaction of specifically designed polymers such as poly $(\beta$-amino esters), cationized cyclodextrins, the biodegradable polymer poly(lactic-co-glycolic) acid (PLGA), combinations between the various polymers prepared by polymer-grafting, or sequencedefined oligomers have also been investigated [163, 164]. From all these classes of polymers, polyethylenimine (existing in branched and linear structures with different molecular weights), a promising candidate for therapeutic siRNA delivery, is one of the most studied cationic polymers. The most prominent commercially available linear PEI is the $22 \mathrm{kDa}$ jetPEI® used as transfection reagent and GMP product available for for clinical studies. The outstanding property of PEI is the high density of which $\sim 20 \%$ that are protonated at physiological $\mathrm{pH}$, allowing efficient complexation of negatively charged nucleic acids at optimal ratios (the so-called nitrogen (in PEI) / phosphate (in RNA) (N/P) ratio) $[165,166]$. The nanoscale PEI polyplexes are able to interact with negatively charged components on the cell membrane leading to nanoparticle internalization via various endocytosis pathways [167]. The "proton sponge effect" of PEI is defined as the capability of PEI to absorb protons during endosomal acidification, resulting in endosome swelling and eventually rupture. This may be a key for the polyplexes to escape the endo-/lysosomal system [168, 169]. During the process, transfection efficacy and biocompatibility depends on the molecular properties and complex preparation conditions. The most suitable PEIs are in the range of $5-25 \mathrm{kD}$, because (1) PEIs with higher molecular weights induce severe cytotoxicity [170], and (2) lower molecular weight PEIs are biologically inactive $[165,171]$. This trend is generally valid for both, linear and branched polymers, despite enhanced tolerance for linear PEIs and better knockdown results with siRNA for branched PEI [172]. Furthermore, several aspects of the preparation conditions may influence the physicochemical and biological properties. Various chemical modifications have been introduced to further improve efficacy and biocompatibility. These include polymer grafting with fatty acids [173, 174], PEG [175-178], amino acids [179-182], and carbohydrates [183, 184], as well as strategies towards targeted delivery by introducing binding ligands to the NP surface. Several modified and non-modified PEIs have been employed in preclinical studies in vivo [183].

Recently, the star polymers for siRNA delivery were designed. These delivery materials contained different lengths of cationic poly(dimethylaminoethyl methacrylate) (PDMAEMA) 
side-arms and varied amounts of poly[oligo(ethylene glycol) methyl ether methacrylate] (POEGMA). They demonstrated that star-POEGMA polymers could readily self-assemble with siRNA to form nanoparticles and deliver siRNA with high efficiency to pancreatic cancer cells [Figure 4 (A)-(B)] [185].

As of 2015, more than 50 clinical trials on RNAi have been conducted or are under way, involving at least 26 different siRNAs. Promising results have been obtained especially for lipidbased siRNA carriers. A cationic lipid formulated with two helper lipids was able to achieve disease stabilization in $52 \%$ of patients with solid tumors [186, 187]. Among the most successful systems "stable nucleic acid lipid particles" (SNALPs), ionizable DlinDMA-lipids were found to be most effective. SNALPs were able to reduce the expression of target genes in hepatocellular carcinoma and metabolic diseases (hypercholesterolemia) $[162,188]$. An alternative approach includes the self-delivery target such as $\mathrm{N}$-acetylgalactosamine-conjugated siRNAs (GalNAcsiRNA) injected subcutaneously. Phase III trials based on GalNAc-siRNA or siRNA formulated in SNALPs are under way for the familial amyloid polyneuropathy (FAP) and familial amyloid cardiomyopathy (FAC) treatment caused by transthyretin (TTR) mutations leading to TTR misfolding and aggregation. In phase I / phase II, a sustained > 80\% knock down of serum TTR has been observed [189]. The other additional systems include siRNA trial using a targeted fourcomponent polymer NP (CALAA-01) for melanoma cancer therapy using a cationized cyclodextrin, adamantane-PEG, adamantane-PEG-transferrin targeted delivery, and the siRNA delivery system. The results of the clinical trials showed that for the patients with solid tumors who were intravenously treated with the NPs, a significant reduction of mRNA and protein levels were achieved. The treatment was first well tolerated but severe adverse effects occurred post 1 year period. NP dissociation /dissolution would have been the main reason upon storage for this adverse effect $[188,190]$. While so far no clinical studies on siRNA-mediated radio-sensitization have been reported, this approach appears clearly feasible based on the preclinical siRNA studies detailed above and the increasing availability of siRNA delivery strategies that can also be employed in clinical studies. Still, efficient and non-toxic siRNA delivery remains a major issue, probably requiring further developments in the NP field.

\section{Structure meets function: carbon nanostructure-based drug delivery}


Carbon Nanostructures including fullerenes, carbon nanotubes (CNTs), carbon nanohorns, nanoribbons, nano-diamonds, and graphene, with various shapes and sizes (e.g. sheets, spheres, ellipsoids, or tubules) [see Figure 5(A)] can have acute biological response via deliberate or undeliberate exposure to the living system [191]. The peculiar physicochemical properties (distinctly different for each structure) play a key role in different research fields including cancer therapy [192, 193]. Among different carbon nanostructures, carbon nanotubes (CNTs) and graphene (GP) derivatives [Figure 5(A)] are known for their use in biomedicine [194-197]. They possess an ordered structure with high aspect ratio, ultralight weight, high mechanical strength, high electrical conductivity, high thermal conductivity, metallic or semi-metallic behavior, and high surface area [198]. Their unique shape allows them to be internalized into cells by penetrating the phospholipid membrane via a "snaking effect"' (spiraling or winding motion) and through transient pores or by active endocytosis ending up in endosomes [199]. These properties make them unique in biomedicines for cancer treatment [200-204]. When using them as drug delivery vehicles, CNT-drug interactions can be mediated by three different mechanisms [205] (1) drug filling inside the CNT channels, (2) absorption of the drug onto the CNT surface and (3) covalent linkage of the drug to the exterior walls [as an example see Figure $5(\mathrm{~B})$ and (C)]. While non-covalent CNT-drug interaction takes place via $\pi-\pi$ stacking, different pathways have been described for covalent attachment of bioactive molecules including acylation and 1,3-dipolar cycloaddition of azomethine ylides reactions [206-210]. As for many other materials, the suitability of CNTs as drug carriers for cancer therapy critically depends on their pharmacokinetic profile goverened by a sufficient transportation to the relevant sites via the blood (intravenous) or lymphatic (subcutaneous and abdominal) circulation. Existing data are not yet conclusive to prove efficacy [209]. Indeed, it is already known that CNTs quickly disperse throughout the body, regardless of the administration site, preferentially accumulate in kidney, stomach and bones with $94 \%$ clearance from the urine and $6 \%$ from biliary pathways, showing potential for carrying drugs and radiotherapeutics to different organ sites and a vital $100 \%$ particle and excess drug clearance [211].

The establishment of CNTs and C-based nanostructures in oncology and radiotherapy requires indepth in vitro and in vivo biological response assessment [212]. Uncertainty of the biological outcomes (positive and negative toxicological effects) for CNT-based materials suggests that using them in drug delivery systems is far reached [213-215]. Furthermore, systemic toxic effects of CNTs such as inflammation, fibrosis, granulomas, and necrosis via strong interference with the 
cellular redox couples also demonstrate the biological hazards post exposure conditions [216]. However, convincing literature data indicate that shorter and thicker CNTs are safer than the longer and thinner ones [217-219]. The application of CNTs for drug delivery and radiotherapy will also require surfacemodifications (material engineering) to improve the hydrophilic behavior and biocompatibility of the material for a changing cellular redox state [220, 221]. Several covalent or non-covalent functionalization approaches are known to optimize biocompatibility of CNTs using natural or chemically synthesized polymeric materials. These materials are active elements for effective delivery vehicles for chemotherapeutics and radio-sensitizing agents [222224]. The manipulation of ROS level by redox modulation via functionalization has been suggested to be feasible for selectively killing cancer cells without any effects to the normal cells [220]. Hence, a key challenge in radiooncology is the development of effective delivery systems for chemotherapeutics, bioactive molecules for ROS generation, or engineering novel materials that reduce the antioxidant defense in the cancer cells. We hypothesize that, amongst others, CNTs allowing controlled release of reactive oxygen to the cancer cell components followed by local irradiation can enhance tumour cell death and treatment outcome [225]. In any case, material engineering and extensive suitability studies are key issues for progress.

Like CNTs, graphene and/or graphene oxide exhibit distinct properties (Figure 5A) [60, 226229]. Graphene is a promising material in medicinal research due to its high cellular interaction and efficient uptake by endocytosis; although a clathrin-dependent endocytosis process has been documented recently for graphene oxide internalization [230], a detailed overview of mechanistic material-cell interactions is not yet possible [231, 232]. Nonetheless, extensive studies focussing on graphene fabrication and its use in tissue scaffolding, bio-imaging, photothermal ablation of malignant cells, and targeted drug delivery have been performed [233, 234]. The physical and chemical interactions such as van der Waals forces and hydrogen bonds are known to be the driving forces for the drug loading in the graphene network [235].

Like CNTs, graphene also has bio-response issues during exposure and might significantly affect normal cells when it is used as drug delivery vehicle. The sources of such responses include graphene edge defects or internal defects of graphene oxide sheets for the generation of massive intracellular ROS [236, 237]. Other cytotoxicity mechanisms involve direct cell membrane damage, depletion of micronutrients, adsorption of nucleic acids, and the DNA intercalation through coordination with chelating ions [238-241]. Pharmacokinetics data show 
graphene/graphene oxide sheets get accumulated in the lungs, liver and spleen, with a much longer retention time (even up to one month) in the lungs, where they induce strong dosedependent inflammatory cell infiltration, pulmonary edema, and granuloma formation [242, 243]. This observation is strictly related to the size, where micro-sized graphene/graphene oxide causes severe inflammation response as compared to $100-350 \mathrm{~nm}$ sheets. In addition, while the graphene/graphene oxides are quickly cleared through the renal routes, the micro-sized particles are preferentially expelled by liver secretion into the biliary tract [60, 244]. It is interesting to note that the graphene surface is immediately covered by biomolecules (proteins, lipids, enzymes) in the cellular interior thus acquiring a new "biological identity" with a dramatic reduction in bio-response profile both in vivo and in vitro [245, 246]. Functionalization of graphene/graphene oxide sheets is performed via strong chemical reactions (nucleophilic and electrophilic substitutions, condensation and addition reaction) between the functional groups (carboxylic, epoxy and/or hydroxyl) and graphene edges, defects, and the basal plane [243, 247]. While covalent and non-covalent graphene systems showed promising drug delivery characteristics, the integration of this research domain with radio-sensitizers for cancer therapy might significantly contribute to the existing state of the art in this field [248, 249].

In summary, CNTs and graphene are promising materials for the use as carriers for chemo- and radio-therapeutics. The intrinsic properties of the carbon surface play a synergistic role in determining biological efficacy and tumor remission. It is clear that the precise synthesis and functionalization of these classes of materials are essential pre-requisites for future studies. Hence, there is a need for fabricating suitable carbon based vehicles possessing high biocompatibility via functionalization with suitable polymeric materials before using them for chemotherapeutic and/or radio-sensitizer delivery in combination therapies.

\section{Polymer functionalization for therapeutics}

In general, the literature shows massive development of nanomaterials and biodegradable delivery systems for improving the efficacy of cancer therapy. Such systems are known for increasing drug solubility in aqueous solution, optimizing pharmacokinetic properties and enhancing intratumoral drug concentration [250]. Other materials recently developed include carbon based materials (discussed earlier) and polymeric materials, protein NPs, organic liposomes, micelles and dendrimers [250, 251]. These nanostructures have been demonstrated as 
delivery vehicles to transport different anticancer agents (chemical drugs, nucleic acids, antibodies and simultaneous multiple drug delivery) to the tumor sites [250, 252]. While the systematic delivery of the drugs to the target sites are now possible, low tumor-to-blood concentration ratio prevents selectivity and triggers severe toxicity in the cells. In contrast, NPs are able to accumulate within the tumor via passive and/or active targeting providing a very high local concentration of the drugs in the tumor tissues. The passive targeting of such NPs relies on the dwelling of these particles within the tumor tissues giving rise to enhanced permeability and retention (EPR) effect (unique feature of the tumor vasculature) [253, 254]. After particle entry and extravasation from the hyperpermeable tumor vessels to the tissue compartment, NPs can increase the local concentration of anticancer drugs by controlled release in the tumor cells. Depending on their sizes, NPs can be internalized by tumor cells through endocytosis mechanisms and passive diffusion across cellular membranes [255-259]. It should be noted that passive tumor targeting might lead to dramatic accumulation of the drugs in tumor sites as high as 5-10 times compared to the free drugs.[260] Furthermore, nanocarrier-based agents have a prolonged half-life compared to the free drugs while simultaneously decreasing systemic toxicity $[255,261,262]$. The representative examples of such delivery systems approved by the FDA (US food and Drug administration) for cancer therapy and the treatment of metastatic breast cancer patients in the European Union include albumin coated NPs for paclitaxel delivery, Abraxane®, poly(ethylene glycol) (PEG) - coated doxorubicin filled liposomes, Doxil@ and non-pegylated liposomal doxorubicin Myocet ${ }^{\circ}[255,263]$. The EPR-dependent delivery of nanocarriers to the tumor sites suffer from several limitations (1) irregular tumor vascularization and poor blood flow inside hypoxic tumors (2) high tumor interstitial fluid pressure impeding the uptake of the particles by tumor cells [264] (3) limitation of the NP internalization due to lack of specific binding motifs to the tumor cells (4) inhomogeneous drug distribution, adsorption and metabolism within the tumor tissues resulting into drug resistance. Hence, the strategic development to overcome these limitations of drug delivery systems would be to efficiently bind NPs to the tumor-specific antibodies or ligands. These targets then bind to the tumor surface receptor inducing receptor-mediated endocytosis releasing the drugs inside the cells. This approach provides high target specificity as well as drug delivery efficiency avoiding drug resistance mechanisms as shown in recent preclinical studies in different types of cancers using these nanocarriers [265-268]. Hence, to overcome the limitations specific functionalized NPs will be designed and will be used in various nano-sensitizers for cancer therapy. The use of 
biocompatible NPs able to deliver nanomedicines at the target cancer sites without affecting normal cells would be an alternative for reducing side effects and biological toxicities. In oncology, polymer therapeutics consist of an anticancer drug covalently bound to a water-soluble macromolecular system from both, natural (e.g. polysaccharides, proteins, peptides) and synthetic polymers (polyethylene glycol, $N$-(2-hydroxypropyl) methacrylamide-HPMA, polyethyleneimine - PEI, poly(L-lysine) - PLL) [269-271]. In principle, the polymer-drug conjugation involves using a biodegradable linker to obtain polymeric prodrug and/or formation of non-cleavable chemical bonds (for examples please see Figure 5C and Figure 6) [272, 273].

To date, different polymer therapeutics are in clinical trial aiming at effective anticancer activity and demonstrating the importance of polymeric moieties and their chemical functionalities [274276]. FDA (US food and Drug administration) approval was obtained for the anti-tumor protein neocarzinostatin conjugated to styrene maleic anhydride (SMANCS), and Pegaspargase or Oncaspar (PEG-L-asparaginase) for the treatment of acute lymphoblastic leukemia [277-280]. The clinical trials also involve polymer conjugates of traditional cytotoxic drugs such as platinates, camptothecin and analogs, paclitaxel/docetaxel, irinotecan, methotrexate, and gemcitabine [132, 281-295]. The concept of water-soluble polymeric-drug conjugates was first proposed by Ringsdorf and hypothesizes about the possibility to modulate either pharmacokinetic profiles of the linked drug, or the site-specificity for the insertion of homing moieties (Figure $5 \mathrm{~B}$ and Figure 6, see covalent and non-covalent linkage) [296, 297]. The conjugated system consists of three different units, (1) a region-device unit for controlling physicochemical properties (2) a drug-linking unit, and (3) an active targeting unit (e.g. monoclonal antibody) allowing for sitespecificity at the cellular level [298, 299].

Conventional anticancer agents suffer from a relatively low therapeutic index and toxic side effects. Hence, due to their low molecular weight, these anticancer agents allow fast clearance from the circulation via renal filtration [283, 300]. Their conjugation to macromolecular systems with improved pharmacokinetic and pharmacodynamics properties [301] can be described as (1) increased solubility in biological fluids [302], (2) increased circulation time in blood [303, 304], as a function of the polymer size [305], (3) decreased toxicity [306], (4) ability to overpass drug resistant mechanism [307], (5) ability to elicit immunostimulatory effects [308, 309], and (6) the possibility to confer active targeting behavior [310, 311]. In addition, polymer therapeutics offer the possibility to combine synergistically radiotherapy and drug targeting [312]. The radio- 
sensitizing effect of a chemotherapeutic agent can be further enhanced by delivering an optimal concentration of the drug maintained in the tumor for a prolonged period [313]. Some key examples of this rationale are poly (L-glutamic acid)-paclitaxel (Xyotax) and albumin-paclitaxel (Abraxane) conjugates. The results demonstrated that the Xyotax was able to reduce the original dose from 53.9 to $7.5 \mathrm{~Gy}$, resulting into $50 \%$ tumor cure in a mouse model. The combination of polymer therapeutics with single dose radiotherapy on ovarian carcinomas showed a dose reduction from 66.6 to 7.9 Gy [314].

In a phase I clinical trial involving 12 patients with localized esophageal and gastric cancer, the same conjugate ( 6 doses; weekly) in combination with fractionated radiotherapy ( 28 cycles; 1.8 Gy daily), allowed four complete responses, and an additional seven partial responses (with reductions in tumor size of more than $50 \%$ ) [315]. In another study, Abraxane (radio-sensitizing agent) exposed to ovarian or mammary carcinoma mouse models showed a reduction of the required dose producing $50 \%$ tumor cures from 54.3 to $35.2 \mathrm{~Gy}$, where the increase in the normal tissue radio-toxicity was not observed [316]. The functionalization and/or coating of polymers on the NP surface allows for coupling of the NP properties with the polymer [317], giving rise to engineered nanohybrids with increased performance for therapeutics oncology or radio-oncology. The interaction of functional nanohybrids (coupling pharmacokinetics and polymer therapeutics) with different human tumors exhibiting various properties including heterogeneity, less pronounced enhanced permeations and retention (EPR) effect, and high propensity of developing resistance to therapies, might increase the performance and reduce the gap between preclinical and clinical human trial data [318], Preliminary data in this direction involve testing of the chemo- and radio-therapeutic efficiency of functional nanohybrids composed of three components comprising (1) polyphenol groups (biologically active component) (2) suitable polymeric materials (biocompatible and stabilizing counterpart), and (3) CNT (cell-interacting element). Results showed that nanohybrid materials with polyphenol groups (bioactive component) suffer from unfavorable pharmacokinetic profiles with low stability in serum and dramatic reduction of in vivo efficiency, despite promising observation in vitro [319, 320].

In a second investigation, polymer-flavonoid conjugates and in particular a dextran-catechin conjugate obtained from enzyme catalysis via free radical reaction was used as therapeutics [321]. Results showed remarkable pharmacokinetic properties, enhanced anticancer activity in pancreatic cancer and neuroblastoma cells, as compared to free flavonoid and superparamagnetic 
NPs (SPIONs) [322-324]. Similarly, the insertion of quercetin into an acrylate polymer chain resulted in a polymeric material with high chemical stability. This therapeutics was used as a component of CNT nanohybrids with high anticancer efficiency and synergistic effect in combination therapy with cisplatin [325-327]. In a pilot study, also anticancer or the radiosensitizing effect of polyphenols was demonstrated, that can be significantly enhanced after incorporation into gelatin-CNT nanohybrids, opening a new research domain in radiooncology $[319,328]$. In the latter case it was shown that combination of irradiation and Catechinnanohybrids can potentially be used for radio-sensitization and eradication of prostate cancer stem cells. A combination of X-ray and treatment with the nanohybrids caused a decrease in the protein level of stem cell-related transcription factors and regulators including Nanog, Oct4, and $\beta$-catenin leading to an increase of cancer cell radio-sensitivity. One may hypothesize about a multifactorial combination therapy involving functional nanohybrids, where the efficiency of suitable chemotherapeutic/radio-sensitizing agents is modulated by means of chemistry, formulations, pharmacokinetics, and biomedicine, to overcome the possible drawback and toxicity concerns enhancing the therapeutic efficiency and safety to reach higher tumor remission rates.

\section{Tumor targeting with nanomaterials}

The literature shows massive development of nanomaterials and biodegradable delivery systems for improving the efficacy of cancer therapy. Such systems are known for increasing drug solubility in aqueous solution, optimizing pharmacokinetic properties and enhancing intratumoral drug concentration [250]. To name few of those material systems recently developed include carbon based materials, polymeric materials, protein NPs, organic liposomes, micelles and dendrimers $[250,251,329]$. These nanostructures have been demonstrated as delivery vehicles to transport different anticancer agents (chemical drugs, nucleic acids, antibodies and simultaneous multiple drug delivery) to the tumor sites [250, 252]. While the systematic delivery of the drugs to the target sites are now possible, low tumor-to-blood concentration ratio prevents selectivity and triggers severe toxicity in the cells. In contrast, NPs are able to accumulate within the tumor via passive and/or active targeting providing a very high local concentration of the drugs in the tumor tissues. The passive targeting of such NPs relies on the dwelling of these NPs within the tumor tissues, tissues giving rise to enhanced permeability and retention (EPR) effect (unique 
feature of the tumor vasculature) $[253,254,330,331]$. After NP entry and extravasation from the hyperpermeable tumor vessels to the tissue compartment, NPs can increase the local concentration of anticancer drugs by controlled release in the tumor cells. Depending on their sizes, NPs can be internalized by tumor cells through endocytosis mechanisms and passive diffusion across cellular membranes [255-259]. It should be noted that passive tumor targeting might lead to dramatic accumulation of the drugs in tumor sites as high as 5-10 times compared to the free drugs [260]. Very recently, it was shown that after initial near-infrared photoimmunotherapy of tumors, the EPR effect is drastically enhanced. This phenomenon is termed super EPR (SUPR) effect [332-334]. Furthermore, nanocarrier-based agents have a prolonged half-life compared to the free drugs while simultaneously decreasing systemic toxicity $[255,261,262]$. The representative examples of such delivery systems approved by the FDA for cancer therapy and the treatment of metastatic breast cancer patients in the European Union include albumin coated NPs for paclitaxel delivery, Abraxane®, poly(ethylene glycol) (PEG) coated doxorubicin filled liposomes, Doxil® and non-pegylated liposomal doxorubicin Myocet® $[255,263]$. The EPR-dependent delivery of nanocarriers to the tumor sites suffer from several limitations as (1) irregular tumor vascularization and poor blood flow inside hypoxic tumors, (2) high tumor interstitial fluid pressure impeding the uptake of the particles by tumor cells [264], (3) limitation of the NP internalization due to lack of specific binding motifs to the tumor cells, and (4) inhomogeneous drug distribution, adsorption and metabolism within the tumor tissues resulting into drug resistance. Hence, the strategic development to overcome these limitations of drug delivery systems would be to efficiently bind NPs to the tumor-specific antibodies or ligands. These targets then bind to the tumor surface receptor inducing receptor-mediated endocytosis releasing the drugs inside the cells. This approach provides high target specificity as well as drug delivery efficiency avoiding drug resistance mechanisms as shown in recent preclinical studies in different types of cancers using these nanocarriers [265-268]. Again, one needs to take care that the ligand actually survive until the NP has reached the tumor site [335], and that they do not increase the NP size too much. Hence, to overcome the limitations one should design specific functionalized NPs and use them in various nano-sensitizers for cancer therapy. The use of biocompatible NPs able to deliver nanomedicines at the target cancer sites without affecting normal cells would be an alternative for reducing side effects and biological toxicities. It is worth mentioning that the intratumoral application of radiolabeled particles is a 
very efficient strategy to destroy cancerous tissues. This is for example demonstrated by the treatment of cancer patients with FDA-approved ${ }^{90}$ Y-containing glass microspheres [336-338].

\section{Promising yet challenging performance: magnetic delivery strategies}

Targeted enrichment of drug-carrying magnetic NPs by the means of internally or externally applied magnetic fields is a promising technique for selective tumor therapy. The basic underlying physical principle hereby is that a magnetic dipole will experience a force in a magnetic field gradient. In this way, magnetic NPs can be directed with magnetic fields [339, 340]. Various magnetic materials on the nanoscale have been developed and applied in different systems. Magnetic implants are mostly deployed, with few exceptions, through blood vessels to guide magnetic drug delivery vehicles. One important approach is intrathecal administration of NPs post deposition of magnetic implants in the subarachnoid space predicted to treat tumors more efficiently via bypasses blood brain barrier [341]. The technique of magnetic implant assisted intravenous application of magnetic NPs offers possibilities for further improvements. The locally generated magnetic field could be enhanced by applying an additional external field to previously implanted micro-ferromagnetic wires. The subsequent NPs administration would lead to enrichments in the targeted area. However, magnetic deposition largely depends on the distance of the applied magnetic field, and therefore it is difficult to be achieved in inner body organs. Currently, some ingenious concepts were introduced to overcome these limitations; nevertheless they are still far reached from realistic clinical application [342, 343]. This is also true for systems which do not rely on magnetic attraction, but on the directional magnetotaxis of drug loaded self-propelling bacteria [344]. The intra-arterial application of $\mathrm{FeO}_{\mathrm{x}} \mathrm{NPs}$ guided by an external electron magnet could be a reasonable route towards clinical application [345]. Those who are developing targeting strategies aiming at clinical translation should follow quality controlled environmental (GXP) protocols, clinically approved technical equipment and pharmaceutically recommended nanoparticles.

Conventional chemotherapy, radiotherapy, or combinations thereof, lack sufficient treatment selectivity in the tumor area, and cause negative effects. Severe short- and long-term side effects can occur including haematologic toxicities, i.e. suppression of bone marrow, as well as nonhematologic adverse effects. This range from dysfunction of liver and kidneys, loss of hair, diarrhea, nausea to reversible but also irreversible skin reactions to lymphedema, tissue fibrosis and even induction of secondary cancers (see for example:[346-351]). One of the future 
therapeutic challenges is thus the development of directed therapy approaches, addressing the tumor more specifically, while sparing the remaining tissues, and increasing the efficiency of the conventional chemotherapeutics or further active ingredients. In this context, nanomedicine in common offers a promising platform for directed applications, capable for reducing the negative side effects of conventional tumor therapy. Regarding the therapeutic applications, drug transportation in the NP-bound form makes even less soluble components available to reach tumor cells [352-354]. A multitude of antitumor drugs, radiotherapeutic nuclides, genetic material, and antibodies can use the NP delivery platform for an improved localized enrichment [355]. Among the materials most commonly used for drug-delivery systems are the NPs or nano shells made of natural or synthetic polymers, as well as metal or metal oxide NPs, such as superparamagnetic magnetic iron oxide NPs (SPIONs).The latter consist of iron oxide cores, often coated with organic materials such as fatty acids, polysaccharides, or polymers [356], to improve colloidal stability and to prevent separation into particles and carrier medium $[357,358]$. The magnetic properties of SPIONs allow remote control of their accumulation by means of external magnetic fields. Conjugation of SPIONs with drugs, in combination with an external magnetic field gradient to target the NPs (so called "Magnetic Drug Targeting", MDT), has additionally emerged as a promising strategy of drug delivery. For the concept of MDT with intraarterial administration of magnetic NPs, an appropriate NP size $(80-150 \mathrm{~nm})$ is important to attract them by means of an external magnet [359]. As demonstrated by in-vivo studies, MDT using mitoxantrone-carrying SPIONs result in increased drug payloads in the target tissue, followed by reducing their systemic dose and toxicity, leading to complete tumor remissions without side effects $[345,360,361]$. Prior to MDT treatments the localization and vascularization of the target tumor is visualized by cone beam flat panel angiography. Beyond that, functional imaging is an attractive tool to recognize target tumor cells in advance. Among the multitude of different possibilities it is worthwhile to especially mention optical coherence tomography (OCT) as useful device for the detection of cancer tissue [362]. Utilizing SPIONs as contrast agents there is another imaging modality called magneto-motive optical Doppler tomography (MMODT) which enables the in-vivo control tumor tissue which is labelled with SPIONs [363]. This would leverage the MDT application since the realtime control of the enrichment of previously applied particles is possible. 
The underlying principle of MDT is illustrated in (Figure 7). Biocompatible-coated SPIONs functionalized with cytostatic agents are administered intraarterially into tumor-supplying vessels, avoiding major capture by the mononuclear phagocyte system (MPS). More precisely, the particles consist of super paramagnetic iron oxide cores, a primery layer of fatty acids as a linker to the second layer albumine, which is the coating shell in which the chemotherapeutic drug mitoxantrone is bound electrostatically. For the direction of the NPs, an electromagnet, with a magnetic field gradient of $72 \mathrm{~T} / \mathrm{m}$ directly at the tip of the pole shoe, was installed [364]. The injection of NPs into the tumor-supplying vessel (arteria femoralis), and simultaneous magnetic field application over a VX2-squamous cell carcinoma placed at the hind limb of the rabbit led to convincing results. An enrichment of SPIONs in the tumor tissue was demonstrated using histology, MRI, and computed-micro- tomography ( $\mu$ CT) [365-367]. Further morphological investigations showed no pathological alterations in liver, kidneys, spleen, lung and brain [364]. The fact that SPIONs in common were usually applied for cancer imaging in liver using MRI, underlines their non-harmfulness. Latest investigations concerning toxicity of SPIONs showed significant effect via surface coating of the nanoparticles. Higher toxicity of SPIONs is related with stronger in situ degradation of the particles and therefore more release of iron ions [368]. In reverse, stabile nanoparticles, like those which applied for MDT, are considered to be safe.

Metabolic decomposition of the SPIONs normally occurs in liver and spleen, in analogy to the physiological iron metabolism. In these organs, iron oxide NPs could detect histologically for three months after application. Radiotracer studies using ${ }^{59} \mathrm{Fe}$ provided evidence that iron from the SPIONs was utilized into the hemoglobin biosynthesis [369] and the cellular uptake of iron oxide [364]. Electron microscopy verified by energy dispersive X-ray analysis visualized the iron oxide NPs inside tumor cells. The therapeutic application of drug-carrying SPIONs (5 \%-10 $\%)$ for the treatment of tumors in rabbits showed complete tumor remissions [360]. This promising outcome has been further confirmed in a large scale study on the application of magnetic NPs in tumor bearing rabbits. The distribution profile after MDT displayed $57.2 \%$ of the total recovery of administered drug, with $66.3 \%$ of the NPs localized in the tumor region, as compared to less than $1 \%$ of drug and NPs reaching the tumor region during conventional intravenous application without magnetic targeting [345]. Angiographic imaging clearly demonstrated that a single MDT application lead to complete and permanent tumor remission. 
MDT could promote the development of super selective and highly efficient tumor therapy approaches. The basic idea of the attempted NP-supported delivery of radio-sensitizers and the respective subsequent irradiation is shown in Figure 8. Each of the applied systems acts on a certain body compartment over a certain time span and only marginally affects the respective region by causing per se just negligible effects in the tissues. Only at the intersection of both spheres, a tremendous increase of cell damage efficacy occurs, inducing tumor destruction. The surface of magnetic NPs can be modified and therefore numerous different radio-sensitizers may be at disposal for MDT. Keeping in mind, NP syntheses should be as complex as necessary but also as simple as possible. This is especially true for the implementation of quality controlled manufacturing concerning current good manufacturing (GMP) guidelines. The stability of the currently deployed SPION carrier has been already proven over time, and a suitable purification strategy is already available. These are preconditions for the overall implementation of a highly localized therapy with minimized side effects [370, 371].

\section{Renal clearable nanomaterials with tumor specific binding as putative radio-sensitizers}

Rapid elimination of nanomaterials containing heavy or toxic metals from the body via urinary system is the desired route for their clearance for minimizing potential health risks originating from nonspecific accumulation and long-term metabolic decomposition in the body. The material properties such as size, surface charge, shape and composition influences the pharmacokinetics of nanomaterials as well as their glomerular filtration [59, 372]. The bio distribution as well as blood residence time of circulating NPs depends fundamentally on their in vivo hydrodynamic diameter that can be substantially larger than their effective in vitro diameter [373]. This is a consequence of unspecific surface adsorption of serum components such as proteins and lipids [374], and in particular agglomeration, due to the presence of high ionic strength [375]. The formation of a biomolecular corona often results in trapping by the MPS and frequently requires adequate surface modifications to prevent this phenomenon [376, 377]. PEGylation for example reduced protein adsorption and thus increases retention times [378, 379]. For a complete analysis, NPs always have to be seen as hybrid systems, involving the actual NP core, a surface coating, and the biomolecular corona [380]. Consequently also the fate of the different NP compounds may vary with time. Retention of NPs including their byproducts in the MPS may trigger an immunological or an inflammatory response, e.g. by intracellular enzymatic breakdown leading 
to the disruption of the protein corona, the surface coating and release of toxic metal ions [335, 381-383]. Many renal clearable nanoparticles with different compositions have been reported and proposed for medicinal applications [383, 384]. In addition to their applications as nanodiagnostics, renal clearable nanomaterials [385], have been discussed as nanotherapeutics for the treatment of diseases, primarily cancer, through photothermal and photodynamic therapy as well as NP-enabled radio-sensitization [383]. Regarding the latter, renal clearable nanomaterials containing chemical elements with a high atomic number (high $Z$ elements) such as gold [386, 387] or gadolinium have been investigated in detail [388-397]. The chelates of such heavy metals, e.g. Gd, functionalized with polysiloxane network have been proposed as theranostic radio-sensitizers due to their (1) facile elimination through the kidneys following intravenous injection in non-tumor bearing mice, and (2) the fact that they accumulate passively in tumors of gliosarcoma bearing rats [252, 392-394, 396, 398]. Irradiation experiments using an orthotopic brain tumor model revealed significant increase in median survival time as compared with untreated animals due to the presence of the gadolinium-based nanomaterials in the brain glioma [393, 394]. It is important to note that the radiation resistance of head and neck squamous cell carcinoma was overcome by the combinational treatment of tumor-bearing mice with these gadolinium containing polysiloxane materials and photon irradiation (Figure 9) [395].

Glutathione-coated gold nanoclusters with core sizes below $2 \mathrm{~nm}$ possess attractive features for clinical use as next generation radio-sensitizers, such as substantial passive tumor accumulation and strong enhancement of external radiotherapy, combined with effective renal elimination and no significant liver or kidney toxicity. Upon intraperitoneal injection into tumor bearing mice, these materials predominantly distribute to tumor and kidneys within $24 \mathrm{~h}$ with low absorption in MPS. The gamma-ray irradiation on the NP- exposed tumors resulted in a substantial decrease of tumor volume and weight $[388,389]$. In all these reports, the radio-sensitizing NPs accumulate passively in the malignant tissues, depending on the pathophysiological vascular architecture of fast growing tumors. In contrast to long circulating NPs, renal clearable agents with short blood retention time are less prone to passive tumor targeting, as they quickly diffuse back to the vasculature and re-enter the systemic circulation. This rapid efflux of passively accumulated NPs from the tumor results only in transient intratumoral presence without substantial retention. The efflux can be diminished by increasing the interactions between NPs and tumor cells and through improvement of cellular uptake. Active or ligand-mediated targeting represents a strategy to 
enable selective recognition of certain membrane receptors or antigens on cancer cells, and to facilitate cellular internalization of NPs through specific interactions such as receptor-mediated endocytosis. A multitude of targeting agents including small molecules, peptides, nucleic acids, as well as proteins, antibodies and their fragments have been investigated for active nanomaterial targeting $[383,399,400]$. These materials have been identified as potential targeting agents for the epidermal growth factor receptor, representing tyrosine kinase overexpressed and/or deregulated in a variety of solid tumors [243, 401-406]. However, the covalent functionalization of nanomaterials with targeting agents is often accompanied by an increase of size and an alteration of surface characteristics [407]. Although extremely exciting and sophisticated ideas have been conceived concerning the application of renal clearable materials in the field of nanomedicine, their clinical translation often proves to be difficult. In order to attain this objective, reproducible and scalable synthesis procedures to obtain precise highly monodisperse and uniform nanomaterials are required [408, 409]. This issue is of fundamental importance for nanomaterials of all sizes as differences in size and shape have a substantial influence on their blood retention time, biodistribution and elimination [383, 410].

\section{Hypothesis-driven development of new NPs for cancer therapy}

Apart from drug delivering nanovehicles, noble metal-based NPs also show potential to increase drug release in the target cell due to enhanced DNA damage and tumor cell death mediated via ROS generation [411-414]. Recently, NPs such as $\mathrm{ZnO}$ and $\mathrm{CuO}$ obtained via flame aerosol technology (FSP) [415-422]. showed striking observations that they ionize in the cellular system, thus posing immediate biohazard (via ROS generation) in the living system (Figure 10 and 11). Dissolution has also been observed for ZnO NPs synthesized with wet-chemistry approaches [423]. The generation of NPs with controllable dissolution kinetics might be a useful therapeutic anticancer agent and under precise conditions of ionic release might selectively kill cancer cells (ongoing work, data not shown). The use of this robust and generic strategy, fine-tuning of the NPs' chemical composition, along with an engineered surface, would enable targeted approaches through intravital administration [424]. Although toxic effects for certain types of NPs have recently been reported, there is still a lack of knowledge for fully understanding their long-term effects in biological systems [425, 426]. The precise designing of engineered NPs (by either reengineering and/or by doping/functionalizing) could have significant impact in the cancer 
therapy [427, 428]. The demonstration of physicochemical properties contributing to hazardous interactions at the nano/bio interface requires advanced techniques to meet these challenges [234, 429-431]. The cellular injury that may be resulting at the membranes, proteins, DNA, organelles, the circulation, and a variety of tissues and organs may show adverse bio-impacts at various oxidative stress levels [414, 432-434]. One approach for countering such impacts for cancer treatment would be to probe such NPs and their wide range of properties using a high-throughput screening platform (a mechanism based approach for screening engineered NPs in vitro for injury) to study their relationship to specific injury responses [435]. The validation of this information at the in vitro level to biological injury in vivo for developing a predictive toxicological paradigm at the biomolecular levels is essential for specific cancer treatments [436438]. The unique properties of engineered NPs warrant safe implementation going beyond traditional hazard, exposure, NP impacts, and risk assessment models to during cancer treatments $[439,440]$. It is known that $\mathrm{ZnO}$ NPs dissolve in the cellular interior and $\mathrm{Zn}^{2+}$ ions make their way to the different organs disrupting the cellular metabolism [see Figure $10(\mathrm{~A})$ ] [409, 430, 441445]. Hence, controlled release of $\mathrm{Zn}^{2+}$ (the concentration needed to chelate the cancer specific components) in cancer cells combined with radio-sensitizers (surface functionalization) might be the unique pathway for cancer treatment. Similarly, a new material based on rare earth metal oxide (REO) also showed significant dissolution profile and triggered cytotoxicity in the cellular environment. The uptake of the NPs in the lysosomes (using macrophage cell-lines) showed pHdependent particle dissolution [Figure 10 (B)]. The released heavy lanthanide ions were chelated by the phosphates from the lysosomes giving rise to deposition of the urchin-shaped crystalline $\mathrm{LaPO}_{4}$. After depleting the phosphate groups of the lysosome, the excess $\mathrm{La}^{3+}$ extracting the phosphate groups of the lysosome membrane leads to cascades of cellular responses such as organelle damage, cathepsin B release, NLRP3 inflammasome activation, and IL-1 $\beta$ release. IL$1 \beta$ is responsible in progressive events including the generation of pro-fibrogenic growth factors by epithelial cells, resulting in pulmonary fibrosis. Hence it is clear that the NPs entering lysosomes or the uptake of the NPs in the cellular interior are prone to dissolution and induction of several biological pathways for hazard generation [431]. The central idea of using this material in cancer treatment is by monitoring of the ionic release of the radio-sensitizer functionalized NPs in the specific cancer cells to increase the reaction kinetics of $\left(\mathrm{PO}_{4}\right)^{3-}$ depletion from the cancer cells without affecting the normal cells (via controlled NP delivery). It is known that the photo-toxicity paradigm (with $\mathrm{Fe}$ doped $\mathrm{TiO}_{2} \mathrm{NPs}$ ) is based on the electrons that are excited to 
the conduction band of $\mathrm{TiO}_{2}$ creating a hole in the valence band via UV light irradiation [446]. The materials that are capable of separating $\mathrm{e}^{-} / \mathrm{h}^{+}$pair in the electronic bands are technologically important, but are critically hazardous to the environment. The $\mathrm{e}^{-} / \mathrm{h}^{+}$pair could then interact with surrounding $\mathrm{H}_{2} \mathrm{O}$ and molecular oxygen to generate $\mathrm{ROS}$ ( $\mathrm{HO}^{\bullet}$ radical and/or superoxide) [429]. The high energetic UV light responsible for such charge separation is a regular obstacle for acquiring knowledge on biological effects through photo activation. Hence, a library of Fe doped $\mathrm{TiO}_{2}$ was developed using flame spray pyrolysis which allowed electronic excitation at lower energy wavelength increasing the cellular apoptosis [429]. The flame aerosol synthesis is a costefficient route to new and functional NPs. The scope of the NPs that can be produced using FSP is much larger due to its utilization of liquid precursors that are directly atomized and ignited forming a spray flame [417, 418, 447, 448]. The liquid precursor, a mixture of organic solvents and metal organic precursors, carries all the energy into the flame. During combustion, nanoparticles grow at very high flame temperatures after the nucleation, surface growth, coagulation and coalescence [422]. The flame aerosol stream is quenched to room temperature with the cold gas [415, 417, 419, 420, 434,449]. The availability of the different metal precursors makes FSP an attractive technique for the synthesis of single and mixed metal oxide particles and their functionalization with noble metals [450, 451]. Using this synthetic knowledge, in-situ mixing of photoluminescence functionalized $\mathrm{Er}^{3+} / \mathrm{Yb}^{3+}$ doped $\mathrm{La}_{2} \mathrm{O}_{3} \mathrm{NPs}_{\text {with }}$ Fe doped $\mathrm{TiO}_{2}$ can easily be produced and allows easy down conversion emission through functionalized rare earth doped $\mathrm{La}_{2} \mathrm{O}_{3}$ with wavelengths exactly equal to the band gap of $\mathrm{Fe}$ doped $\mathrm{TiO}_{2}$ NPs [452]. The emitted light (in the near visible range, and thus harmless to tissue) will excite electrons in the Fe doped $\mathrm{TiO}_{2} \mathrm{NPs}$ and interact with the cancer cells. These combined effects can be exploited to trace the particles in the cells (due to its violet colour emission) at a specific location for possible interaction with the cancer cells to produce ROS species (Figure 12). The paradigm described here might have significant development towards cancer therapy but requires precise materials designing and characterization.

\section{Demanding Intermediate: Preclinical Models for Nanoparticle Evaluation}

In recent years, nano-modified drugs have shown improved material chemistry such as solubility, pharmacokinetics, and bio-distribution compared to small molecules [453]. NP based drugs can 
be effective at lower doses making cancer treatment more economic and minimizing side effects. However, the design of such effective NP based drugs for translation into the clinics requires extensive and careful in vivo evaluation. The use of appropriate preclinical models of human cancers is key to this process. Models of choice should (1) resemble the pathophysiological and microenvironmental characteristics of the malignant disease of interest for target identification and/or validation, (2) allow to study pharmacodynamic/pharmacokinetic properties of novel NPs of interest, and (3) be suitable for exploring the utility of functionalized NPs to synergize with radiotherapeutic treatment.

Various animal models are available and should be carefully considered for NP testing based on their specific properties and the analytical endpoint(s) of interest. These include:

- Ectopic xenograft models - human cancer cells (primary or established cell lines) or tumor biopsy material injected or implanted (subcutaneously, intraperitoneally, intravenously, and/or intramuscularly) into syngeneic or immune-suppressed hosts.

- Orthotopic xenograft models - human cancer cells (primary or established cell lines) transplanted into the host organ that corresponds to their tissue of origin

- Carcinogen-induced models - animal tumors developing spontaneously after exposure to chemical or radiation stimuli

- Germ-line transgenic and conditional transgenic models (GEMMs) - animal tumors developing upon tissue- and temporal-specific regulation of specific (human-relevant) oncogenes or tumor suppressor genes

Further models of interest based on the above but with particular characteristics are:

- Primary human tumor grafts (implanted ectopically or orthotopically) - xenograft is expected to have the identical genotypic profile as the primary human tumor

- Humanized xenograft models - animal for human cancer cell engraftment (ectopically or orthotopically) is manipulated to develop human-like immune responses.

Ectopic xenografts are a valuable tool for the assessment of nano-drugs, radiotherapy, and other therapeutic components exploited for specific cancer treatments. In principle, these models have been used to study and quantify dose responses along with tumor pharmacodynamics [454]. Subcutaneous cancer cell implantation for example, allows to monitor tumor growth via simple calliper measurements and are easy-handling and efficient for demonstrating biodistribution and treatment response. So far, subcutaneous xenografts are the only feasible model for the assessment of the radiotherapeutically-relevant curative analytical endpoint tumor control dose 
50 as the radiation dose required to cure $50 \%$ of tumor-bearing mice. This value should be lowered upon combination with radiosensitizing (drug) moieties [455]. Nonetheless, despite promising results in the literature, ectopic models have several disadvantages such as (1) irregular tumor growth in the host and loss of heterogeneity [456], (2) limited reflection of primary genetic profiles, clinical outcome and pathophysiological characteristics, [457], and (3) difficulty in studying angiogenesis induction and metastasis.

In orthotopic cancer models, explants of the primary tumor cell are injected to the tissue of the malignancy origin. Results from this model are realistic and show enhanced metastatic rates of human cancer progression [458]. It is important to note that immune-compromised animals or humanized mice are absolutely essential for the injection and engraftment of human cancer cells [459].Comparing orthotopic with ectopic xenograft models, the former have the possibility to develop an organotypic microenvironment, recapitulate the local milieu, and study the role of organ-specific cell-stromal interactions on tumor growth and metastasis [138]. These models have been successfully applied in preclinical trials to evaluate drug-dose combinations and animal survival [460]. Major drawbacks are the high variation in the tumor development (due to animal morbidities during orthotopic surgical implantation) and the requirement of sophisticated imaging for tumor detection and monitoring.

Animal tumors (Carcinogen-induced models and GEMMs) are highly relevant for mechanistic studies and immune response monitoring. Examples of such well known carcinogen-induced preclinical models in immune-competent mice and rat include: NMU ( $N$-nitroso- $N$-methylurea)induced mammary carcinoma, DEN (diethylnitrosamine)-induced hepatocellular carcinoma, and NMU- and MNNG ( $N$-methyl- $N$-nitro- $N$-nitrosoguanidine)-induced gastric carcinoma models [461-463]. These models are key in determining the progression and stage during treatment and evaluation of suitable preventative interventions with therapeutic agents. However, latest improvements in genetic engineering have particularly extended the panel of transgenic (genetically engineered mouse tumor) models and broadened their use for demonstration of complex biological processes and therapy testing in the presence of a mature immune system. Transgenic and genetically engineered mouse tumor models directly relate to the dysregulation of oncogenes and tumor suppressor genes. They are histologically and genetically comparable or even similar to human cancers but tumors often develop asynchronously which can be problematic for standardization of treatment testing. Most of the models show low penetrance and/or latency in tumor development and growth suffering from the strict requirement of 
exclusive tissue-specific promoters regulating the transgene expression [464]. Notably, novel approaches for the development of conditionally regulated transgenic models with high penetrance properties and clinical relevance have recently been described [465]. As for orthotopic models, all of these organ-specific animal tumors require sophisticated imaging technology, and application of clinically relevant fractionated radiotherapy regimes for combination treatment testing will not be possible without an advanced 3-D animal treatment planning strategy.

Patient-derived tumor xenografts (PDTX) are innovative fast emerging models for preclinical trials in cancer treatment. PDTX develop from primary human tumor material excised within a few hours of surgery and grafted into immune-deficient mice preserving the genotypic and phenotypic features of the original tumor. Literature data imply a 30-40\% failure rate for engraftment of implants, and successfully implanted material requires several months (6-8) for in vivo propagation via serial transplantation [466]. While this model looks particularly promising with respect to physiological and clinical relevance for therapy response prediction, quality assessment of the freshly excised primary tumor tissue is difficult, estimation of engraftment success is thus not possible and handling remains expensive and time-consuming. In any case, PDTX are delicate models for systematic and extended treatment test programs and also restrict the study of immunotherapies, i.e. anti-cancer agents that target components of the immune system cannot be studied. In this context, humanized xenograft models might be the next generation model.

Humanized xenograft models are obtained via co-engrafting tumor fragments with human peripheral blood/bone marrow cells into NOD/SCID mice and allowing reconstitution of the murine immune system. This approach enables to (1) resemble human immune responses in a mouse model, (2) monitor the impact of human immune cells on tumor progression and metastasis and (3) study the impact of immune modulators and drugs directed against or stimulating the human immune system [467].

Different models might be exploited to verify the potential use of nanomaterials for anti-cancer treatment. Indeed, due to the limitations and advantages of each model, exposure of the same nano-drug in several animal models might be required to evaluate its in-vivo activity, pharmacokinetics and bio-responses alone and in combination with irradiation However, as stated by Ruggeri et al. in their highly informative review on pre-clinical animal models of cancer [456]: "ultimate proof of concept for efficacy and safety of novel oncology therapeutics lies in 
humans" This is off course true for any nano-radiosensitizer approaches and the concepts highlighted herein.

\section{Conclusion}

While the standard radio/chemotherapy can kill the bulk tumor cells, even a small population of surviving malignant cells (radio-resistant and putatively multi-drug resistant cancer cells) can result in tumor recurrence and metastasis. Hence, the use of specially-designed, reengineered NPs for radiooncology is proposed to critically contribute to the sensitization of all tumor cells, including the disastrous radio-resistant population, thereby improving the cure of cancer by biologically individualized radiotherapy [Figure 13]. For future use of NPs in radiooncology a potential comprehensive strategy as discussed in this report would focus on (1) precise NP synthesis for radiooncology and cancer therapy, (2) functionalization of these NPs using radiosensitizers, (3) biological response assessment using conventional and sophisticated in-vitro and in-vivo models, and (4) pre-clinical and clinical testing for treatment outcome. Future objectives in developing NPs for cancer treatment should lie on the clinical utilization of radio-sensitizing strategies based on (1) the optimized delivery of known and novel radio-sensitizing drugs, in particular therapeutic small RNA molecules for radio-sensitization (no report yet), and (2) manufacturing of nanomaterials that can be activated during state-of-the-art individualized radiotherapy [Figure $13(\mathrm{~A})$ and $(\mathrm{B})]$.

The multidisciplinary expertise will allow for exploring a multifactorial combination therapy involving functional nanohybrids, where the efficiency of suitable therapeutic, radio-sensitizing agents is modulated by means of chemical formulations, pharmacokinetics, and biomedicine to overcome the possible drawback and toxicity concerns enhancing the therapeutic efficiency and safety to reach high tumor remission rate. The combination of NP research, their functionalization towards radiooncology and clinical trials is one of the potential research domains and directions for curing cancer in the $21^{\text {st }}$ century.

\section{Acknowledgements}

(1) LAKS, $\mathrm{AD}$ and $\mathrm{CP}$ would like to thank the funding support from BMBF grants (ZIK OncoRay) (2) MHM and AA thank for the generous support of the "Rudolf Ernst Stiftung für Gesundheitswesen" (Rudolf Ernst für Gesundheitswesen Geschäftszeichen 6/15) (3) HS and KZ thank for the financial support by the Helmholtz Virtual Institute NanoTracking (Agreement no. 
VH-VI-421) as well as the research initiative Technologie und Medizin - Multimodale Bildgebung zur Aufklärung des in-vivo-Verhaltens von polymeren Biomaterialien of the Helmholtz-Portfoliothema (4) OV acknowledges the kind support of a Cancer Institute New South Wales Early Career Fellowship (13/ECF/1-01). MK thanks the National Health and Medical Research Council (NHMRC) for a Senior Research Fellowship (APP1058299) and Program Grant (APP1091261); Australian Research Council Centre of Excellence in Convergent Bio-Nano Science and Technology (CE140100036) and a Cancer Council New South Wales Program Grant (PG16-01) (5) WJP would like to thank the support of the German Research Foundation (DFG grant PA 794/25-1) for the parts of this work. (6) SP and LM would like to thank for the support from the US Public Health Service (Grant, RO1 ES016746). The work also leveraged the infrastructure that is supported by the National Science Foundation and the Environmental Protection Agency under Cooperative Agreement Number DBI 0830117 and 1266377

\section{Figure Captions}

Figure 1. Schematic illustration of key intracellular tumor radioresistance mechanism and possible therapeutic intervention for radio-sensitization. Ionizing radiation is inducing single (SSB) and double strand breaks (DSB) within the DNA by direct or indirect effects via the generation of free radicals. These DNA damages are recognized by DNA repair machinery proteins, e.g. phosphorylated histone $\mathrm{H} 2$ isoform $\mathrm{AX}(\gamma \mathrm{H} 2 \mathrm{AX})$, protein kinase ataxiatelangiectasia mutated (ATM), ataxia telangiectasia, Rad3-related serine/threonin protein kinase (ATR), and DNA-dependent protein kinase (DNA-PK). High numbers of unrepaired residual DNA breaks lead to tumor cell death. The efficacy of radiotherapy is affected by three main tumor radio-resistance mechanisms (1) enhanced DNA repair capacity, (2) tumor hypoxia (low levels of oxygen $\left(\mathrm{O}_{2}\right)$ accompanied by reduced reactive oxygen species (ROS) due to the pathophysiological vascular network in the tumor tissue, and (3) hyperactivation of cell survival signaling such as the phosphoinositide 3-kinase (PI3K), mitogen-activated protein kinase (MAPK), nuclear factor kappa-light-chain-enhancer of activated B cells (NFkB) pathways and/or inactivation of the p53 tumor suppressor gene. Therapeutic interventions interfering with these mechanisms as exemplified herein can induce tumor radio-sensitization and may enhance the curative potential of radiotherapy upon combinatorial treatment..

Figure 2: (A) Overview of various (nanoparticle) systems for the delivery of RNAi-inducing agents [468]. For details, see text. (B) The complex nanoparticle systems rely on chemical surface modifications, aiming at reduced non-specific interactions with blood components or non-target tissues, improved pharmacokinetics (biodistribution, clearance/excretion, and 
circulation halflife), enhanced biocompatibility and, in case of a coupling ligand, and targeted delivery. Beyond siRNAs, other chemically modified or non-modified RNA molecules, or other oligonucleotides, can be delivered as well. (C) Scheme of PEI-based nanoparticle (polyplex) formation, based on the electrostatic interaction between PEI and siRNAs. By combining the polyplexes with liposomes, lipopolyplexes are formed. Upon their endocytosis, the polyplexes or lipopolyplexes are released from the endosomal/lysosomal system due to the so-called protonsponge effect, prior to release of the siRNA from the nanoparticle and the siRNA-induced target gene knockdown (for details, see text). Right panel: silencing of EGFP in stable reporter cell lines upon PEI-mediated delivery of siEGFP (lower panel) vs. negative control siRNA (upper panel). (D) (Lipo-) polyplexes have also been explored for therapeutic siRNA delivery in vivo. Upon systemic injection of lipopolyplexes, intact siRNAs are delivered into various organs including tumor xenografts, as indicated by the bands in gel electrophoresis/autoradiograhy representing full-length, [32] P-labeled siRNAs (upper right). In a subcutaneous tumor xenograft model (lower left), treatment with siRNAs targeting the anti-apoptotic proto-oncogene survivin (siSurv) leads to marked tumor-inhibitory effects, as compared to the negative control treatment group (siCtrl). This inhibition of tumor growth is based on target gene knockdown, as determined from the survivin levels in the tumors upon termination of the experiment (lower right). (A) Adapted with permission, copyright (2015) Elsevier Science. (D) Adapted with permission, copyright (2016) Elsevier Science.

Figure 3: Examples of liposomal or polymeric systems suitable for siRNA delivery. Polymers like PEI are available as branched or linear molecules (upper panels), and branched polymer structures also include dendrimers (upper right). DOTMA and DOTAP represent examples of older lipids, while SNALPs, comprising of the components shown in the lower right, have later on been developed for siRNA delivery in vivo.

Figure 4. High power magnified cation confocal microscopic images of MiaPaCa-2 cells transfected with star 3-siRNA showing nternalized siRNA had no or less colocalization (A) Systemic administration of star-POEGMA-siRNA gave rise to enhanced siRNA accumulation in pancreatic tumors in mice. siRNA binding efficiency and cell uptake of star-POEGMA-siRNA complexes in pancreatic cancer cells (B) Confocal microscope images demonstrating cell uptake of fluorescently labeled-siRNA (green shown by arrow) [185]. Adapted with permission, copyright (2008) American Chemical Society

Figure 5 (A) Representation of carbon nanostructures. [242] (B) Single-walled carbon nanotubes (SWNTs) may present antibodies with a higher local density to stimulate $\mathrm{T}$ cells to release Interleukin-2 (IL-2). The schematic representation (not to the scale) shows the anti-CD3adsorbed CNT inducing T-cell (B3Z cells) stimulation. (C) Representation of CNTs used as a drug carrier. Cisplatin is covalently ligated to surface-oxidized CNTs as an effective anti-tumor agent, and a folic acid molecule is further coupled to the cisplatin as a targeting molecule. The large surface area of CNTs makes it possible to carry more cisplatins into tumor cells. Adapted with permission, copyright (2016) Elsevier, copyright (2008) American Chemical Society [242, 469, 470].

Figure 6. Putative chemical modifications of nanoscale materials (carbon nanotubes are shown as example) result in functionalized moieties that can interact with the surrounding cellular 
components. Upper panel: Aromatic molecules functionalized on the particle surface via $\pi-\pi$ interactions (non-covalent interaction). Middle panel: Polymers are wrapped around the carbon based NPs by non-covalent interactions (including $\pi-\pi$, van der Waals forces and charge-transfer interactions. Lower panel: Chemical groups that covalently bind to the surface react with styrene monomers to form polystyrene chains. Adapted with permission from nature publishing group copyright (2007) [471].

Figure 7: Principle of Magnetic Drug Targeting (MDT). Left side: Drug-carrying magnetic nanoparticles are injected intra-arterially close to the tumor. An electromagnet positioned in the tumor area directs and attracts the iron oxide particles in the tumor region. Right side: the particles themselves consist of the super-paramagnetic iron oxide core, a primary layer of fatty acids as biocompatible spacer and a second layer of albumin. The chemotherapeutic agent mitoxantrone is bound electrostatically inside the albumine layer.

Figure 8. A recognized tumor will be treated by Magnetic Drug Targeting in order to enrich radio-sensitizers which are enriched by NPs in the tumor area, followed by irradiating to induce neutron capture to destroy cells only in the region of boron or gadolinium accumulation.

Figure 9: Simplified scheme comparing the biodistribution of NPs. Renal clearable nanomaterials (blue) and their larger counterparts (green) differ substantially in blood retention time and route of excretion. The former can be eliminated rapidly from the circulatory system via bladder and urine after passing the glomerular filtration. Clearance of nanomaterials bypassing the renal filtration occurs mainly in the liver, where their metabolic decomposition and biodegradation take place. Both subsets of NPs can passively or actively target malignant tissues, whereas the later approach requires their functionalization with appropriate targeting moieties.

Figure 10. Particle dissolution mechanism in the cells (A) $\mathrm{ZnO}$ dissolution in the materialbiocomponent interface and lysosome generates cellular toxicity through the release of $\mathrm{Zn}^{2+}$ ions inducing a cascades of harmful cellular events such as lysosomal damage, mitochondrial perturbation, ROS production, excitation of pro-inflammatory cytokine and chemokine production. (B) Cellular mechanisms showing pro-fibrogenic effects via rare earth oxides exposure. The uptake of RE oxides by macrophages and lysosomes damage cell organelles lead to IL-1 $\beta$ production causing pulmonary fibrosis. The lower cellular model shows molecular mechanism where phospholipids are dephosphorylated causing crystalline $\mathrm{REPO}_{4}$ to precipitate on the surface. Adapted with permission from nature publishing group copyright (2009) and ACS copyright (2014) (ACS Editors' Choice article) [409, 431].

Figure 11. Flames spray pyrolysis (FSP) technique for NP synthesis. Left panel: The schematic diagram of the FSP reactor. Right panel: a photograph of a roaring flame during the NP production.

Figure 12. The combination of down conversion emission followed by near visible excitation for the $\mathrm{e}^{-} / \mathrm{h}^{+}$pair separation using CNT non/functionalized $\mathrm{Er}^{3+} / \mathrm{Yb}^{3+}$ doped $\mathrm{La}_{2} \mathrm{O}_{3}$ in-situ mixed with $\mathrm{Fe}$ doped $\mathrm{TiO}_{2}$ an application which could be effectively tested in the cells.

Figure 13 (A) Schematic illustration of NP-based mechanisms for tumor radio-sensitization. Fast growing tumors depend on constant supply of nutrients and hence are able to induce vascular 
sprouting, leading to an irregular vascular network inside the tumor (neoangiogenesis). In these tumors, the vascular network efficiently captures NPs, resulting into an accumulation in the tumor by several uptake mechanisms such as phagocytosis, pinocytosis and receptor/clathrinmediated endocytosis. After internalization, endosomal escape may take place, followed by nuclear transport to release drugs (B) Schematic illustration of NP-based treatment modalities for tumor radio--sensitization. Standard cancer therapy is including a combination of radio- and chemotherapy. This treatment is reducing the tumor volume by killing tumor bulk cells, whereas radio-resistant tumor cells cells survive, regrow the tumor, leading to tumor relapse. The combination of radio/chemotherapy with NP-based systems including radio-sensitizing agents has the potential to sensitize the radio-resistant population and eradicate the tumor bulk cells together with the radio-resistant cells. Tumor control and patient cure can only be achieved when all tumor cells are killed.

\section{References}

[1] J. Ferlay, I. Soerjomataram, R. Dikshit, S. Eser, C. Mathers, M. Rebelo, D.M. Parkin, D. Forman, F. Bray, Cancer incidence and mortality worldwide: sources, methods and major patterns in GLOBOCAN 2012, Int. J. Cancer 136(5) (2015) E359-86.

[2] C. Fitzmaurice, D. Dicker, A. Pain, H. Hamavid, M. Moradi-Lakeh, M.F. MacIntyre, C. Allen, G. Hansen, R. Woodbrook, C. Wolfe, R.R. Hamadeh, A. Moore, A. Werdecker, B.D. Gessner, B. Te Ao, B. McMahon, C. Karimkhani, C. Yu, G.S. Cooke, D.C. Schwebel, D.O. Carpenter, D.M. Pereira, D. Nash, D.S. Kazi, D. De Leo, D. Plass, K.N. Ukwaja, G.D. Thurston, K. Yun Jin, E.P. Simard, E. Mills, E.K. Park, F. Catala-Lopez, G. deVeber, C. Gotay, G. Khan, H.D. Hosgood, 3rd, I.S. Santos, J.L. Leasher, J. Singh, J. Leigh, J. Jonas, J. Sanabria, J. Beardsley, K.H. Jacobsen, K. Takahashi, R.C. Franklin, L. Ronfani, M. Montico, L. Naldi, M. Tonelli, J. Geleijnse, M. Petzold, M.G. Shrime, M. Younis, N. Yonemoto, N. Breitborde, P. Yip, F. Pourmalek, P.A. Lotufo, A. Esteghamati, G.J. Hankey, R. Ali, R. Lunevicius, R. Malekzadeh,

R. Dellavalle, R. Weintraub, R. Lucas, R. Hay, D. Rojas-Rueda, R. Westerman, S.G. Sepanlou, S. Nolte, S. Patten, S. Weichenthal, S.F. Abera, S.M. Fereshtehnejad, I. Shiue, T. Driscoll, T. Vasankari, U. Alsharif, V. Rahimi-Movaghar, V.V. Vlassov, W.S. Marcenes, W. Mekonnen, Y.A. Melaku, Y. Yano, A. Artaman, I. Campos, J. MacLachlan, U. Mueller, D. Kim, M. Trillini, B. Eshrati, H.C. Williams, K. Shibuya, R. Dandona, K. Murthy, B. Cowie, A.T. Amare, C.A. Antonio, C. Castaneda-Orjuela, C.H. van Gool, F. Violante, I.H. Oh, K. Deribe, K. Soreide, L. Knibbs, M. Kereselidze, M. Green, R. Cardenas, N. Roy, T. Tillman, Y. Li, H. Krueger, L. Monasta, S. Dey, S. Sheikhbahaei, N. Hafezi-Nejad, G.A. Kumar, C.T. Sreeramareddy, L. Dandona, H. Wang, S.E. Vollset, A. Mokdad, J.A. Salomon, R. Lozano, T. Vos, M. Forouzanfar, A. Lopez, C. Murray, M. Naghavi, The Global Burden of Cancer 2013, JAMA Oncol. 1(4) (2015) 505-27.

[3] K. Pushpavanam, E. Narayanan, J. Chang, S. Sapareto, K. Rege, A Colorimetric Plasmonic Nanosensor for Dosimetry of Therapeutic Levels of Ionizing Radiation, ACS nano 9(12) (2015) 11540-50.

[4] W.C.W. Chan, B. Udugama, P. Kadhiresan, J. Kim, S. Mubareka, P.S. Weiss, W.J. Parak, Patients, Here Comes More Nanotechnology, ACS nano (2016).

[5] C.A. Schütz, L. Juillerat-Jeanneret, H. Mueller, I. Lynch, M. Riediker, Therapeutic nanoparticles in clinics and under clinical evaluation, Nanomed. 8(3) (2013) 449-467. 
[6] S. Wilhelm, A.J. Tavares, Q. Dai, S. Ohta, J. Audet, H.F. Dvorak, W.C.W. Chan, Analysis of nanoparticle delivery to tumours, Nat. Rev. Mater. 1 (2016) 16014.

[7] Y.A. Lau, D.P. Ferris, J.I. Zink, Photo-driven nano-impellers and nanovalves for oncommand drug release, 2010, pp. 75740P-75740P-10.

[8] M. Liong, J. Lu, M. Kovochich, T. Xia, S.G. Ruehm, A.E. Nel, F. Tamanoi, J.I. Zink, Multifunctional Inorganic Nanoparticles for Imaging, Targeting, and Drug Delivery, ACS nano 2(5) (2008) 889-896.

[9] D. Tarn, C.E. Ashley, M.I.N. Xue, E.C. Carnes, J.I. Zink, C.J. Brinker, Mesoporous Silica Nanoparticle Nanocarriers - Biofunctionality and Biocompatibility, Accounts of chemical research 46(3) (2013) 792-801.

[10] C.R. Thomas, D.P. Ferris, J.-H. Lee, E. Choi, M.H. Cho, E.S. Kim, J.F. Stoddart, J.-S. Shin, J. Cheon, J.I. Zink, Noninvasive Remote-Controlled Release of Drug Molecules in Vitro Using Magnetic Actuation of Mechanized Nanoparticles, Journal of the American Chemical Society 132(31) (2010) 10623-10625.

[11] Y. Zhang, Z. Hou, Y. Ge, K. Deng, B. Liu, X. Li, Q. Li, Z. Cheng, P.a. Ma, C. Li, J. Lin, DNA-Hybrid-Gated Photothermal Mesoporous Silica Nanoparticles for NIR-Responsive and Aptamer-Targeted Drug Delivery, ACS Applied Materials \& Interfaces 7(37) (2015) 2069620706.

[12] Z. Ali, A.Z. Abbasi, F. Zhang, P. Arosio, A. Lascialfari, M.F. Casula, A. Wenk, W. Kreyling, R. Plapper, M. Seidel, R. Niessner, J. Knöll, A. Seubert, W.J. Parak, Multifunctional Nanoparticles for Dual Imaging, Anal. Chem. 83(8) (2011) 2877-2882.

[13] D.E.J.G.J. Dolmans, D. Fukumura, R.K. Jain, Photodynamic therapy for cancer, Nat Rev Cancer 3(5) (2003) 380-387.

[14] C.D. Cole, J.K. Liu, X. Sheng, S.S. Chin, M.H. Schmidt, M.H. Weiss, W.T. Couldwell, Hypericin-mediated photodynamic therapy of pituitary tumors: preclinical study in a GH4C1 rat tumor model, Journal of Neuro-Oncology 87(3) (2008) 255-261.

[15] T. Jean-philippe, F. Celine, M. Vincent, B.-H. Muriel, Phthalocyanines Covalently Bound to Biomolecules for a Targeted Photodynamic Therapy, Current Medicinal Chemistry 14(15) (2007) 1673-1687.

[16] R. Minnes, H. Weitman, Y. You, M.R. Detty, B. Ehrenberg, Dithiaporphyrin Derivatives as Photosensitizers in Membranes and Cells, The Journal of Physical Chemistry B 112(10) (2008) 3268-3276.

[17] J.L. Sessler, R.A. Miller, Texaphyrins: New drugs with diverse clinical applications in radiation and photodynamic therapy, Biochemical pharmacology 59(7) (2000) 733-739.

[18] J. Trachtenberg, A. Bogaards, R.A. Weersink, M.A. Haider, A. Evans, S.A. McCluskey, A. Scherz, M.R. Gertner, C. Yue, S. Appu, A. Aprikian, J. Savard, B.C. Wilson, M. Elhilali, Vascular Targeted Photodynamic Therapy With Palladium-Bacteriopheophorbide Photosensitizer for Recurrent Prostate Cancer Following Definitive Radiation Therapy: Assessment of Safety and Treatment Response, The Journal of Urology 178(5) (2007) 1974-1979.

[19] A. Vargas, M. Eid, M. Fanchaouy, R. Gurny, F. Delie, In vivo photodynamic activity of photosensitizer-loaded nanoparticles: Formulation properties, administration parameters and biological issues involved in PDT outcome, European Journal of Pharmaceutics and Biopharmaceutics 69(1) (2008) 43-53.

[20] K.K. Wang, L. Lutzke, L. Borkenhagen, W. Westra, M.W.K. Song, G. Prasad, N.S. Buttar, Photodynamic therapy for Barrett's esophagus: does light still have a role?, Endoscopy 40(12) (2008) 1021-1025. 
[21] A. Ferrario, F.v.K. Tiehl, N. Rucker, A.M. Schwarz, G.S. P., J.G.J. C., Antiangiogenic Treatment Enhances Photodynamic Therapy Responsiveness in a Mouse Mammary Carcinoma, Cancer Research 60(15) (2000) 4066 - 4069.

[22] H.S. de Bruijn, N.v.d. Veen, D.J. Robinson, W.M. Star, Improvement of Systemic 5Aminolevulinic Acid-based Photodynamic Therapy in Vivo Using Light Fractionation with a 75Minute Interval Cancer Research 59(4) (1999) 901.

[23] D.E.J.G.J. Dolmans, A. Kadambi, J.S. Hill, K.R. Flores, J.N. Gerber, J.P. Walker, I.H.M.B. Rinkes, R.K. Jain, D. Fukumura, Targeting Tumor Vasculature and Cancer Cells in Orthotopic Breast Tumor by Fractionated Photosensitizer Dosing Photodynamic Therapy, Cancer Research 62(15) (2002) 4289.

[24] Y. Cheng, A. C. Samia, J.D. Meyers, I. Panagopoulos, B. Fei, C. Burda, Highly Efficient Drug Delivery with Gold Nanoparticle Vectors for in Vivo Photodynamic Therapy of Cancer, Journal of the American Chemical Society 130(32) (2008) 10643-10647.

[25] J. Wang, R. Deng, M.A. MacDonald, B. Chen, J. Yuan, F. Wang, D. Chi, T.S. Andy Hor, P. Zhang, G. Liu, Y. Han, X. Liu, Enhancing multiphoton upconversion through energy clustering at sublattice level, Nat Mater 13(2) (2014) 157-162.

[26] R. Atun, D.A. Jaffray, M.B. Barton, F. Bray, M. Baumann, B. Vikram, T.P. Hanna, F.M. Knaul, Y. Lievens, T.Y. Lui, M. Milosevic, B. O'Sullivan, D.L. Rodin, E. Rosenblatt, J. Van Dyk, M.L. Yap, E. Zubizarreta, M. Gospodarowicz, Expanding global access to radiotherapy, Lancet. Oncol. 16(10) (2015) 1153-86.

[27] M. Baumann, M. Krause, J. Overgaard, J. Debus, S.M. Bentzen, J. Daartz, C. Richter, D. Zips, T. Bortfeld, Radiation oncology in the era of precision medicine, Nat. Rev. Cancer 16(4) (2016) 234-249.

[28] A.C. Begg, F.A. Stewart, C. Vens, Strategies to improve radiotherapy with targeted drugs, Nat. Rev. Cancer 11(4) (2011) 239-53.

[29] S.M. Bentzen, Preventing or reducing late side effects of radiation therapy: radiobiology meets molecular pathology, Nat. Rev. Cancer 6(9) (2006) 702-13.

[30] M. Krause, A. Dubrovska, A. Linge, M. Baumann, Cancer stem cells: Radioresistance, prediction of radiotherapy outcome and specific targets for combined treatments, Advanced drug delivery reviews.

[31] C. Peitzsch, I. Kurth, L. Kunz-Schughart, M. Baumann, A. Dubrovska, Discovery of the cancer stem cell related determinants of radioresistance, Radiother. Oncol. 108(3) (2013) 378-87. [32] J.-P. Pouget, I. Navarro-Teulon, M. Bardies, N. Chouin, G. Cartron, A. Pelegrin, D. Azria, Clinical radioimmunotherapy[mdash]the role of radiobiology, Nat. Rev. Clin. Oncol. 8(12) (2011) 720-734.

[33] J.M. Slater, Considerations in identifying optimal particles for radiation medicine, Technol. Cancer Res. Treat. 5(2) (2006) 73-9.

[34] P. Harald, Relative biological effectiveness (RBE) values for proton beam therapy. Variations as a function of biological endpoint, dose, and linear energy transfer, Physics in medicine and biology 59(22) (2014) R419-R472.

[35] M.C. Joiner, A.J. Van der Kogel, t.E. chapter 24 in Basic Clinical Radiobiology, pp68-77, ( Eds. Joiner MC and Van der Kogel AJ), CRC Press, Taylor \& Francis Group, LLC, Boca Raton, FL, USA, Energy transfer and relative biological effectiveness in protons and other ions in radiotherapy,, CRC Press, Taylor \& Francis Group, LLC, Boca Raton, FL, USA Chapter 24, Basic Clinical Radiobiology,(4th Edition) (2009).

[36] F. Tommasino, M. Durante, Proton Radiobiology, Cancers 7(1) (2015). 
[37] F. Tommasino, E. Scifoni, M. Durante, New Ions for Therapy, International Journal of Particle Therapy 2(3) (2015) 428-438.

[38] U. Weber, G. Kraft, Comparison of Carbon Ions Versus Protons, The Cancer Journal 15(4) (2009) 325-332.

[39] U. Amaldi, G. Kraft, Radiotherapy with beams of carbon ions, Reports on Progress in Physics 68(8) (2005) 1861-1882.

[40] D. Wang, A critical appraisal of the clinical utility of proton therapy in oncology, Medical devices 8 (2015) 439-46.

[41] E.S. Wisenbaugh, P.E. Andrews, R.G. Ferrigni, S.E. Schild, S.R. Keole, W.W. Wong, S.A. Vora, Proton beam therapy for localized prostate cancer 101: basics, controversies, and facts, Rev. Urol. 16(2) (2014) 67-75.

[42] M. Uhl, K. Herfarth, J. Debus, Comparing the use of protons and carbon ions for treatment, Cancer J. 20(6) (2014) 433-9.

[43] E. Mladenov, S. Magin, A. Soni, G. Iliakis, DNA double-strand break repair as determinant of cellular radiosensitivity to killing and target in radiation therapy, Front. Oncol. 3 (2013) 113.

[44] N. Dhani, A. Fyles, D. Hedley, M. Milosevic, The clinical significance of hypoxia in human cancers, Semin. Nucl. Med 45(2) (2015) 110-21.

[45] P. Vaupel, A. Mayer, Hypoxia in tumors: pathogenesis-related classification, characterization of hypoxia subtypes, and associated biological and clinical implications, Adv. Exp. Med. Biol. 812 (2014) 19-24.

[46] C. Peitzsch, R. Perrin, R.P. Hill, A. Dubrovska, I. Kurth, Hypoxia as a biomarker for radioresistant cancer stem cells, Int. J. Rad. Biol. 90(8) (2014) 636-52.

[47] M.L. Mierzwa, M.K. Nyati, M.A. Morgan, T.S. Lawrence, Recent advances in combined modality therapy, Oncol. 15(4) (2010) 372-81.

[48] W. Sihver, J. Pietzsch, M. Krause, M. Baumann, J. Steinbach, H.J. Pietzsch, Radiolabeled Cetuximab Conjugates for EGFR Targeted Cancer Diagnostics and Therapy, Pharmaceuticals 7(3) (2014) 311-38.

[49] M.A. Morgan, T.S. Lawrence, Molecular Pathways: Overcoming Radiation Resistance by Targeting DNA Damage Response Pathways, Clin. Cancer Res. 21(13) (2015) 2898-2904.

[50] D.R. Wahl, M.H. Stenmark, Y. Tao, E.L. Pollom, E.M. Caoili, T.S. Lawrence, M.J. Schipper, M. Feng, Outcomes After Stereotactic Body Radiotherapy or Radiofrequency Ablation for Hepatocellular Carcinoma, J. Clin. Oncol. 34(5) (2016) 452-459.

[51] M. Venere, P. Hamerlik, Q. Wu, R.D. Rasmussen, L.A. Song, A. Vasanji, N. Tenley, W.A. Flavahan, A.B. Hjelmeland, J. Bartek, J.N. Rich, Therapeutic targeting of constitutive PARP activation compromises stem cell phenotype and survival of glioblastoma-initiating cells, Cell Death and Differentiation 21(2) (2014) 258-269.

[52] C.V.M. Verhagen, R. de Haan, F. Hageman, T.P.D. Oostendorp, A.L.E. Carli, M.J. O'Connor, J. Jonkers, M. Verheij, M.W. van den Brekel, C. Vens, Extent of radiosensitization by the PARP inhibitor olaparib depends on its dose, the radiation dose and the integrity of the homologous recombination pathway of tumor cells, Radiother. Oncol. 116(3) (2015) 358-365.

[53] F.P. Vendetti, A. Lau, S. Schamus, T.P. Conrads, M.J. O'Connor, C.J. Bakkenist, The orally active and bioavailable ATR kinase inhibitor AZD6738 potentiates the anti-tumor effects of cisplatin to resolve ATM-deficient non-small cell lung cancer in vivo, Oncotarget 6(42) (2015) 44289-44305.

[54] S.N. Sanghavi, M.N. Needle, M.D. Krailo, J.R. Geyer, J. Ater, M.P. Mehta, A phase I study of topotecan as a radiosensitizer for brainstem glioma of childhood: First report of the Children's Cancer Group-0952, Neuro-Oncol. 5(1) (2003) 8-13. 
[55] K.K. Ang, Q. Zhang, D.I. Rosenthal, P.F. Nguyen-Tan, E.J. Sherman, R.S. Weber, J.M. Galvin, J.A. Bonner, J. Harris, A.K. El-Naggar, M.L. Gillison, R.C. Jordan, A.A. Konski, W.L. Thorstad, A. Trotti, J.J. Beitler, A.S. Garden, W.J. Spanos, S.S. Yom, R.S. Axelrod, Randomized Phase III Trial of Concurrent Accelerated Radiation Plus Cisplatin With or Without Cetuximab for Stage III to IV Head and Neck Carcinoma: RTOG 0522, J. Clin. Oncol. 32(27) (2014) 29402950.

[56] J.A. Bonner , P.M. Harari , J. Giralt , N. Azarnia , D.M. Shin , R.B. Cohen , C.U. Jones , R. Sur , D. Raben , J. Jassem , R. Ove , M.S. Kies , J. Baselga , H. Youssoufian , N. Amellal , E.K. Rowinsky, K.K. Ang Radiotherapy plus Cetuximab for Squamous-Cell Carcinoma of the Head and Neck, New Engl. J. Med. 354(6) (2006) 567-578.

[57] M.W. Epperly, S.Y. Lai, A.J. Kanai, N. Mason, B. Lopresi, T. Dixon, D. Franicola, Y. Niu, W.R. Wilson, J.S. Greenberger, Effectiveness of Combined Modality Radiotherapy of Orthotopic Human Squamous Cell Carcinomas in $\mathrm{Nu} / \mathrm{Nu}$ Mice Using Cetuximab, Tirapazamine and MnSOD-Plasmid Liposome Gene Therapy, In vivo 24(1) (2010) 1-8.

[58] P.M. Harari, J. Harris, M.S. Kies, J.N. Myers, R.C. Jordan, M.L. Gillison, R.L. Foote, M. Machtay, M. Rotman, D. Khuntia, W. Straube, Q. Zhang, K. Ang, Postoperative Chemoradiotherapy and Cetuximab for High-Risk Squamous Cell Carcinoma of the Head and Neck: Radiation Therapy Oncology Group RTOG-0234, J. Clin. Oncol. 32(23) (2014) 24862495.

[59] J. Liu, M. Yu, C. Zhou, J. Zheng, Renal clearable inorganic nanoparticles: a new frontier of bionanotechnology, Materials Today 16(12) (2013) 477-486.

[60] K. Wang, J. Ruan, H. Song, J. Zhang, Y. Wo, S. Guo, D. Cui, Biocompatibility of Graphene Oxide, Nanoscale Res. Lett. 6(1) (2011) 1-8.

[61] J. Giralt, J. Trigo, S. Nuyts, M. Ozsahin, K. Skladowski, G. Hatoum, J.-F. Daisne, A.C. Yunes Ancona, A. Cmelak, R. Mesía, A. Zhang, K.S. Oliner, A. VanderWalde, Panitumumab plus radiotherapy versus chemoradiotherapy in patients with unresected, locally advanced squamous-cell carcinoma of the head and neck (CONCERT-2): a randomised, controlled, openlabel phase 2 trial, Lancet Oncol. 16(2) (2015) 221-232.

[62] R. Mesía, M. Henke, A. Fortin, H. Minn, A.C. Yunes Ancona, A. Cmelak, A.B. Markowitz, S.J. Hotte, S. Singh, A.T.C. Chan, M.C. Merlano, K. Skladowski, A. Zhang, K.S. Oliner, A. VanderWalde, J. Giralt, Chemoradiotherapy with or without panitumumab in patients with unresected, locally advanced squamous-cell carcinoma of the head and neck (CONCERT-1): a randomised, controlled, open-label phase 2 trial, Lancet Oncol. 16(2) (2015) 208-220.

[63] K.G. Babu, K. Prabhash, A.K. Vaid, B. Sirohi, R.B. Diwakar, R. Rao, M. Kar, H. Malhotra, S. Nag, C. Goswami, V. Raina, R. Mohan, Nimotuzumab plus chemotherapy versus chemotherapy alone in advanced non-small-cell lung cancer: a multicenter, randomized, openlabel Phase II study, OncoTargets Ther. 7 (2014) 1051-1060.

[64] J. Liang, M. E, G. Wu, L. Zhao, X. Li, X. Xiu, N. Li, B. Chen, Z. Hui, J. Lv, H. Fang, Y. Tang, N. Bi, W. Wang, Y. Zhai, T. Li, D. Chen, S. Zou, N. Lu, R. Perez-Rodríguez, J. Zheng, L. Wang, Nimotuzumab combined with radiotherapy for esophageal cancer: preliminary study of a Phase II clinical trial, OncoTargets Therapy 6 (2013) 1589-1596.

[65] M.T. Solomón, J.C. Selva, J. Figueredo, J. Vaquer, C. Toledo, N. Quintanal, S. Salva, R. Domíngez, J. Alert, J.J. Marinello, M. Catalá, M.G. Griego, J.A. Martell, P.L. Luaces, J. Ballesteros, N. de-Castro, F. Bach, T. Crombet, Radiotherapy plus nimotuzumab or placebo in the treatment of high grade glioma patients: results from a randomized, double blind trial, BMC Cancer 13(1) (2013) 1-8. 
[66] N.K. Gerber, Y. Yamada, A. Rimner, W. Shi, G.J. Riely, K. Beal, H.A. Yu, T.A. Chan, Z. Zhang, A.J. Wu, Erlotinib Versus Radiation Therapy for Brain Metastases in Patients With EGFR-Mutant Lung Adenocarcinoma, Int. J. Rad. Oncol. Biol. Phys. 89(2) (2014) 322-329.

[67] Y. Jiang, H.B. Mackley, E.T. Kimchi, J. Zhu, N. Gusani, J. Kaifi, K.F. Staveley-O’Carroll, C.P. Belani, Phase I dose escalation study of capecitabine and erlotinib concurrent with radiation in locally advanced pancreatic cancer, Cancer Chemother. Pharmacol. 74(1) (2014) 205-210.

[68] R.G. Martins, U. Parvathaneni, J.E. Bauman, A.K. Sharma, L.E. Raez, M.A. Papagikos, F. Yunus, B.F. Kurland, K.D. Eaton, J.J. Liao, E. Mendez, N. Futran, D.X. Wang, X. Chai, S.G. Wallace, M. Austin, R. Schmidt, D.N. Hayes, Cisplatin and Radiotherapy With or Without Erlotinib in Locally Advanced Squamous Cell Carcinoma of the Head and Neck: A Randomized Phase II Trial, J. Clin. Oncol. 31(11) (2013) 1415-1421.

[69] M.D. Prados, S.M. Chang, N. Butowski, R. DeBoer, R. Parvataneni, H. Carliner, P. Kabuubi, J. Ayers-Ringler, J. Rabbitt, M. Page, A. Fedoroff, P.K. Sneed, M.S. Berger, M.W. McDermott, A.T. Parsa, S. Vandenberg, C.D. James, K.R. Lamborn, D. Stokoe, D.A. HaasKogan, Phase II Study of Erlotinib Plus Temozolomide During and After Radiation Therapy in Patients With Newly Diagnosed Glioblastoma Multiforme or Gliosarcoma, J. Clin. Oncol. 27(4) (2009) 579-584.

[70] T.A.T. Fong, L.K. Shawver, L. Sun, C. Tang, H. App, T.J. Powell, Y.H. Kim, R. Schreck, X. Wang, W. Risau, A. Ullrich, K.P. Hirth, G. McMahon, SU5416 Is a Potent and Selective Inhibitor of the Vascular Endothelial Growth Factor Receptor (Flk-1/KDR) That Inhibits Tyrosine Kinase Catalysis, Tumor Vascularization, and Growth of Multiple Tumor Types, Cancer Res. 59(1) (1999) 99-106.

[71] M.G. Fury, A. Zahalsky, R. Wong, E. Venkatraman, E. Lis, L. Hann, T. Aliff, W. Gerald, M. Fleisher, D.G. Pfister, A Phase II study of SU5416 in patients with advanced or recurrent head and neck cancers, Investig. New Drugs 25(2) (2007) 165-172.

[72] A. Stopeck, M. Sheldon, M. Vahedian, G. Cropp, R. Gosalia, A. Hannah, Results of a Phase I dose-escalating study of the antiangiogenic agent, SU5416, in patients with advanced malignancies, J. Am. Assoc. Cancer Res. 8 (2002) 2798-2805.

[73] P.G. Corn, D.Y. Song, E. Heath, J. Maier, R. Meyn, D. Kuban, T.A. DePetrillo, P. Mathew, Sunitinib plus androgen deprivation and radiation therapy for patients with localized high-risk prostate cancer: results from a multi-institutional phase I study, Int. J. Rad. Oncol. Biol. Phys. 86(3) (2013) 540-545.

[74] G.D. Demetri, A.T. van Oosterom, C.R. Garrett, M.E. Blackstein, M.H. Shah, J. Verweij, G. McArthur, I.R. Judson, M.C. Heinrich, J.A. Morgan, J. Desai, C.D. Fletcher, S. George, C.L. Bello, X. Huang, C.M. Baum, P.G. Casali, Efficacy and safety of sunitinib in patients with advanced gastrointestinal stromal tumour after failure of imatinib: a randomised controlled trial, Lancet 368(9544) 1329-1338.

[75] J. Lewin, K.K. Khamly, R.J. Young, C. Mitchell, R.J. Hicks, G.C. Toner, S.Y.K. Ngan, S. Chander, G.J. Powell, A. Herschtal, L. Te Marvelde, J. Desai, P.F.M. Choong, S.A. Stacker, M.G. Achen, N. Ferris, S. Fox, J. Slavin, D.M. Thomas, A phase Ib/II translational study of sunitinib with neoadjuvant radiotherapy in soft-tissue sarcoma, Br. J. Cancer 111(12) (2014) 2254.

[76] C.C.L. Tong, E.C. Ko, M.W. Sung, J.A. Cesaretti, R.G. Stock, S.H. Packer, K. Forsythe, E.M. Genden, M. Schwartz, K.H.V. Lau, M. Galsky, J. Ozao-Choy, S.-H. Chen, J. Kao, Phase II Trial of Concurrent Sunitinib and Image-Guided Radiotherapy for Oligometastases, PloS one 7(6) (2012) e36979. 
[77] E.J. Wuthrick, M. Kamrava, W.J. Curran, M. Werner-Wasik, K.A. Camphausen, T. Hyslop, R. Axelrod, D.W. Andrews, J. Glass, M. Machtay, A.P. Dicker, A phase Ib trial of the combination of the anti-angiogenic agent sunitinib and radiation therapy for patients with primary and metastatic central nervous system malignancies, Cancer 117(24) (2011) 5548-5559.

[78] P. Chinnaiyan, M. Won, P.Y. Wen, A.M. Rojiani, M. Wendland, T.A. Dipetrillo, B.W. Corn, M.P. Mehta, RTOG 0913: A Phase I Study of Daily Everolimus (RAD001) In Combination with Radiation Therapy and Temozolomide in Patients with Newly Diagnosed Glioblastoma, Int. J. Rad. Oncol. Biol. Phys. 86(5) (2013) 880-884.

[79] E. Deutsch, C. Le Péchoux, L. Faivre, S. Rivera, Y. Tao, J.-P. Pignon, M. Angokai, R. Bahleda, D. Deandreis, E. Angevin, C. Hennequin, B. Besse, A. Levy, J.-C. Soria, Phase I trial of everolimus in combination with thoracic radiotherapy in non-small-cell lung cancer, Ann. Oncol. 26(6) (2015) 1223-1229.

[80] M.G. Fury, N.Y. Lee, E. Sherman, A.L. Ho, S. Rao, A. Heguy, R. Shen, S. Korte, D. Lisa, I. Ganly, S. Patel, R.J. Wong, A. Shaha, J. Shah, S. Haque, N. Katabi, D.G. Pfister, A Phase 1 Study of Everolimus + Weekly Cisplatin + Intensity Modulated Radiation Therapy in Head-andNeck Cancer, Int. J. Rad. Oncol. Biol. Phys. 87(3) (2013) 479-486.

[81] D.J. Ma, E. Galanis, S.K. Anderson, D. Schiff, T.J. Kaufmann, P.J. Peller, C. Giannini, P.D. Brown, J.H. Uhm, S. McGraw, K.A. Jaeckle, P.J. Flynn, K.L. Ligon, J.C. Buckner, J.N. Sarkaria, A phase II trial of everolimus, temozolomide, and radiotherapy in patients with newly diagnosed glioblastoma: NCCTG N057K, Neuro-Oncol. 17(9) (2015) 1261-1269.

[82] O.L. Chinot, W. Wick, W. Mason , R. Henriksson , F. Saran , R. Nishikawa , A.F. Carpentier, K. Hoang-Xuan, P. Kavan, D. Cernea , A.A. Brandes , M. Hilton , L. Abrey , T. Cloughesy Bevacizumab plus Radiotherapy-Temozolomide for Newly Diagnosed Glioblastoma, New Engl. J. Med. 370(8) (2014) 709-722.

[83] B.G. Czito, J.C. Bendell, C.G. Willett, M.A. Morse, G.C. Blobe, D.S. Tyler, J. Thomas, K.A. Ludwig, C.R. Mantyh, J. Ashton, D. Yu, H.I. Hurwitz, Bevacizumab, Oxaliplatin, and Capecitabine With Radiation Therapy in Rectal Cancer: Phase I Trial Results, Int. J. Rad. Oncol. Biol. Phys. 68(2) (2007) 472-478.

[84] A. Lai, A. Tran, P.L. Nghiemphu, W.B. Pope, O.E. Solis, M. Selch, E. Filka, W.H. Yong, P.S. Mischel, L.M. Liau, S. Phuphanich, K. Black, S. Peak, R.M. Green, C.E. Spier, T. Kolevska, J. Polikoff, L. Fehrenbacher, R. Elashoff, T. Cloughesy, Phase II Study of Bevacizumab Plus Temozolomide During and After Radiation Therapy for Patients With Newly Diagnosed Glioblastoma Multiforme, J. Clin. Oncol. 29(2) (2011) 142-148.

[85] M. Verstraete, A. Debucquoy, J. Dekervel, J. van Pelt, C. Verslype, E. Devos, G. Chiritescu, K. Dumon, A. D'Hoore, O. Gevaert, X. Sagaert, E. Van Cutsem, K. Haustermans, Combining bevacizumab and chemoradiation in rectal cancer. Translational results of the AXEBeam trial, Br. J. Cancer 112(8) (2015) 1314-1325.

[86] S.-W. Chen, L.-C. Lin, Y.-C. Kuo, J.-A. Liang, C.-C. Kuo, J.-F. Chiou, Phase 2 Study of Combined Sorafenib and Radiation Therapy in Patients With Advanced Hepatocellular Carcinoma, Int. J. Rad. Oncol. Biol. Phys. 88(5) (2014) 1041-1047.

[87] E.G. Chiorean, B.P. Schneider, F.M. Akisik, S.M. Perkins, S. Anderson, C.S. Johnson, J. DeWitt, P. Helft, R. Clark, E.L. Johnston, A.J. Spittler, J. Deluca, G. Bu, S. Shahda, P.J. Loehrer, K. Sandrasegaran, H.R. Cardenes, Phase 1 Pharmacogenetic and Pharmacodynamic Study of Sorafenib With Concurrent Radiation Therapy and Gemcitabine in Locally Advanced Unresectable Pancreatic Cancer, Int. J. Rad. Oncol. Biol. Phys. 89(2) (2014) 284-291.

[88] A.F. Hottinger, A.B. Aissa, V. Espeli, D. Squiban, N. Dunkel, M.I. Vargas, T. Hundsberger, N. Mach, K. Schaller, D.C. Weber, A. Bodmer, P.Y. Dietrich, Phase I study of sorafenib 
combined with radiation therapy and temozolomide as first-line treatment of high-grade glioma, Br. J. Cancer 110(11) (2014) 2655-2661.

[89] A.B. Lassman, S.L. Pugh, M.R. Gilbert, K.D. Aldape, S. Geinoz, J.H. Beumer, S.M. Christner, R. Komaki, L.M. DeAngelis, R. Gaur, E. Youssef, H. Wagner, M. Won, M.P. Mehta, Phase 2 trial of dasatinib in target-selected patients with recurrent glioblastoma (RTOG 0627), Neuro-Oncol. 17(7) (2015) 992-998.

[90] T.B. Brunner, M. Geiger, G.G. Grabenbauer, M. Lang-Welzenbach, T.S. Mantoni, A. Cavallaro, R. Sauer, W. Hohenberger, W.G. McKenna, Phase I Trial of the Human Immunodeficiency Virus Protease Inhibitor Nelfinavir and Chemoradiation for Locally Advanced Pancreatic Cancer, J. Clin. Oncol. 26(16) (2008) 2699-2706.

[91] J. Buijsen, G. Lammering, R.L.H. Jansen, G.L. Beets, J. Wals, M. Sosef, M.O. Den Boer, J. Leijtens, R.G. Riedl, J. Theys, P. Lambin, Phase I trial of the combination of the Akt inhibitor nelfinavir and chemoradiation for locally advanced rectal cancer, Radiother. Oncol. 107(2) (2013) 184-188.

[92] E.J. Hill, C. Roberts, J.M. Franklin, M. Enescu, N. West, T.P. MacGregor, K.-Y. Chu, L. Boyle, C. Blesing, L.-M. Wang, S. Mukherjee, E.M. Anderson, G. Brown, S. Dutton, S.B. Love, J.A. Schnabel, P. Quirke, R. Muschel, W.G. McKenna, M. Partridge, R.A. Sharma, Clinical Trial of Oral Nelfinavir before and during Radiation Therapy for Advanced Rectal Cancer, Am. Ass. Cancer Res. 22(8) (2016) 1922-1931.

[93] J.M. Wilson, E. Fokas, S.J. Dutton, N. Patel, M.A. Hawkins, C. Eccles, K.-Y. Chu, L. Durrant, A.G. Abraham, M. Partridge, M. Woodward, E. O’Neill, T. Maughan, W.G. McKenna, S. Mukherjee, T.B. Brunner, ARCII: A phase II trial of the HIV protease inhibitor Nelfinavir in combination with chemoradiation for locally advanced inoperable pancreatic cancer, Radiother. Oncol. 119(2) (2016) 306-311.

[94] N. Butowski, S.M. Chang, K.R. Lamborn, M.Y. Polley, R. Pieper, J.F. Costello, S. Vandenberg, R. Parvataneni, A. Nicole, P.K. Sneed, J. Clarke, E. Hsieh, B.M. Costa, R.M. Reis, M. Hristova-Kazmierski, S.J. Nicol, D.E. Thornton, M.D. Prados, Phase II and pharmacogenomics study of enzastaurin plus temozolomide during and following radiation therapy in patients with newly diagnosed glioblastoma multiforme and gliosarcoma, NeuroOncol. 13(12) (2011) 1331-1338.

[95] B.H. Grønberg, T. Ciuleanu, Ø. Fløtten, A. Knuuttila, E. Abel, S.W. Langer, K. Krejcy, A.M. Liepa, M. Munoz, M. Hahka-Kemppinen, S. Sundstrøm, A placebo-controlled, randomized phase II study of maintenance enzastaurin following whole brain radiation therapy in the treatment of brain metastases from lung cancer, Lung Cancer 78(1) (2012) 63-69.

[96] W. Wick, J.P. Steinbach, M. Platten, C. Hartmann, F. Wenz, A. von Deimling, P. Shei, V. Moreau-Donnet, C. Stoffregen, S.E. Combs, Enzastaurin before and concomitant with radiation therapy, followed by enzastaurin maintenance therapy, in patients with newly diagnosed glioblastoma without MGMT promoter hypermethylation, Neuro-Oncol. 15(10) (2013) 14051412.

[97] M.A. Hassan Metwally, R. Ali, M. Kuddu, T. Shouman, P. Strojan, K. Iqbal, R. Prasad, C. Grau, J. Overgaard, IAEA-HypoX. A randomized multicenter study of the hypoxic radiosensitizer nimorazole concomitant with accelerated radiotherapy in head and neck squamous cell carcinoma, Radiother. Oncol. 116(1) (2015) 15-20.

[98] J.M. Henk, K. Bishop, S.F. Shepherd, Treatment of head and neck cancer with CHART and nimorazole: phase II study, Radiother. Oncol. 66(1) (2003) 65-70.

[99] J. Overgaard, H. Sand Hansen, M. Overgaard, L. Bastholt, A. Berthelsen, L. Specht, B. Lindeløv, K. Jørgensen, A randomized double-blind phase III study of nimorazole as a hypoxic 
radiosensitizer of primary radiotherapy in supraglottic larynx and pharynx carcinoma. Results of the Danish Head and Neck Cancer Study (DAHANCA) Protocol 5-85, Radiother. Oncol. 46(2) (1998) 135-146.

[100] J.L.H. Johnson, R.A. Leos, A.F. Baker, E.C. Unger, Radiosensitization of Hs-766T Pancreatic Tumor Xenografts in Mice Dosed with Dodecafluoropentane NanoEmulsion\&\#8211;Preliminary Findings, J. Biomed. Nanotechnol. 11(2) (2015) 274-281.

[101] J.L. Gainer, J.P. Sheehan, J.M. Larner, D.R. Jones, Trans sodium crocetinate with temozolomide and radiation therapy for glioblastoma multiforme, J. Neurosurgr. 0(0) 1-7.

[102] J.P. Sheehan, M.E. Shaffrey, B. Gupta, J. Larner, J.N. Rich, D.M. Park, Improving the radiosensitivity of radioresistant and hypoxic glioblastoma, Future Oncol. 6(10) (2010) 1591601.

[103] F.E. Lock, P.C. McDonald, Y. Lou, I. Serrano, S.C. Chafe, C. Ostlund, S. Aparicio, J.Y. Winum, C.T. Supuran, S. Dedhar, Targeting carbonic anhydrase IX depletes breast cancer stem cells within the hypoxic niche, Oncogene 32(44) (2013) 5210-5219.

[104] Y. Lou, P.C. McDonald, A. Oloumi, S. Chia, C. Ostlund, A. Ahmadi, A. Kyle, U. auf dem Keller, S. Leung, D. Huntsman, B. Clarke, B.W. Sutherland, D. Waterhouse, M. Bally, C. Roskelley, C.M. Overall, A. Minchinton, F. Pacchiano, F. Carta, A. Scozzafava, N. Touisni, J.-Y. Winum, C.T. Supuran, S. Dedhar, Targeting Tumor Hypoxia: Suppression of Breast Tumor Growth and Metastasis by Novel Carbonic Anhydrase IX Inhibitors, Cancer Res. 71(9) (2011) 3364-3376.

[105] C. Ward, J. Meehan, P. Mullen, C. Supuran, J.M. Dixon, J.S. Thomas, J.-Y. Winum, P. Lambin, L. Dubois, N.-K. Pavathaneni, E.J. Jarman, L. Renshaw, I. Um, C. Kay, D.J. Harrison, I.H. Kunkler, S.P. Langdon, Evaluation of carbonic anhydrase IX as a therapeutic target for inhibition of breast cancer invasion and metastasis using a series of in vitro breast cancer models, Oncotarget 6(28) (2015) 24856-24870.

[106] Y. Ogawa, K. Kubota, H. Ue, M. Tadokoro, R. Matsui, T. Yamanishi, N. Hamada, S. Kariya, A. Nishioka, H. Nakajima, M. Tarutani, S. Sano, , Safety and effectiveness of a new enzyme-targeting radiosensitization treatment (KORTUC II) for intratumoral injection for lowLET radioresistant tumors

Int. J. Oncol. 39(3) (2011) 553-560.

[107] M. Mohammadianpanah, S. Razmjou-Ghalaei, A. Shafizad, Y. Ashouri-Taziani, B. Khademi, N. Ahmadloo, M. Ansari, S. Omidvari, A. Mosalaei, M. Mosleh-Shirazi, Efficacy and safety of concurrent chemoradiation with weekly cisplatin \&\#177; low-dose celecoxib in locally advanced undifferentiated nasopharyngeal carcinoma: A phase II-III clinical trial, J. Cancer Res. Therapeutics 7(4) (2011) 442-447.

[108] L.-W. Wang, C.-F. Hsiao, W.T.-L. Chen, H.-H. Lee, T.-C. Lin, H.-C. Chen, H.-H. Chen, C.-R. Chien, T.-Y. Lin, T.-W. Liu, Celecoxib plus chemoradiotherapy for locally advanced rectal cancer: A phase II TCOG study, J. Surgr. Oncol. 109(6) (2014) 580-585.

[109] W. Shi, J.D. Palmer, M. Werner-Wasik, D.W. Andrews, J.J. Evans, J. Glass, L. Kim, V. Bar-Ad, K. Judy, C. Farrell, N. Simone, H. Liu, A.P. Dicker, Y.R. Lawrence, Phase I trial of panobinostat and fractionated stereotactic re-irradiation therapy for recurrent high grade gliomas, J. Neurooncol. 127(3) (2016) 535-539.

[110] H.S. Takhar, N. Singhal, R. Gowda, M. Penniment, P. Takhar, M.P. Brown, Phase I study evaluating the safety and efficacy of oral panobinostat in combination with radiotherapy or chemoradiotherapy in patients with inoperable stage III non-small-cell lung cancer, Anti-Cancer Drugs 26(10) (2015) 1069-1077. 
[111] A. Avallone, M.C. Piccirillo, P. Delrio, B. Pecori, E. Di Gennaro, L. Aloj, F. Tatangelo, V. D’Angelo, C. Granata, E. Cavalcanti, N. Maurea, P. Maiolino, F. Bianco, M. Montano, L. Silvestro, M. Terranova Barberio, M.S. Roca, M. Di Maio, P. Marone, G. Botti, A. Petrillo, G. Daniele, S. Lastoria, V.R. Iaffaioli, G. Romano, C. Caracò, P. Muto, C. Gallo, F. Perrone, A. Budillon, Phase 1/2 study of valproic acid and short-course radiotherapy plus capecitabine as preoperative treatment in low-moderate risk rectal cancer-V-shoRT-R3 (Valproic acid - short RadioTherapy - rectum 3rd trial), BMC Cancer 14(1) (2014) 1-12.

[112] A.V. Krauze, S.D. Myrehaug, M.G. Chang, D.J. Holdford, S. Smith, J. Shih, P.J. Tofilon, H.A. Fine, K. Camphausen, A Phase 2 Study of Concurrent Radiation Therapy, Temozolomide, and the Histone Deacetylase Inhibitor Valproic Acid For Patients With Glioblastoma, Int. J. Rad. Oncol. Biol. Phys. 92(5) (2015) 986-992.

[113] D.G. Brachman, S.L. Pugh, L.S. Ashby, T.A. Thomas, E.M. Dunbar, S. Narayan, H.I. Robins, J.A. Bovi, J.K. Rockhill, M. Won, W.P. Curran, Phase I/II Trial of Temozolomide (TMZ), Motexafin Gadolinium (MGd), and 60 Gy Fractionated Radiation (RT) for Newly Diagnosed Supratentorial Glioblastoma Multiforme (GBM): Final Results of RTOG 0513, Int. J. Rad. Oncol. Biol. Phys. 91(5) (2015) 961-967.

[114] J.M. Ford, W. Seiferheld, J.R. Alger, G. Wu, T.J. Endicott, M. Mehta, W. Curran, S.-C. Phan, Results of the Phase I Dose-Escalating Study of Motexafin Gadolinium With Standard Radiotherapy in Patients With Glioblastoma Multiforme, Int. J. Rad. Oncol. Biol. Phys. 69(3) (2007) 831-838.

[115] W.N. William Jr, R.G. Zinner, D.D. Karp, Y.W. Oh, B.S. Glisson, S.-C. Phan, D.J. Stewart, Phase I Trial of Motexafin Gadolinium in Combination with Docetaxel and Cisplatin for the Treatment of Non-small Cell Lung Cancer, J. Thorac. Oncol. 2(8) (2007) 745-750.

[116] L.L. Rojas-Puentes, M. Gonzalez-Pinedo, A. Crismatt, A. Ortega-Gomez, C. GamboaVignolle, R. Nuñez-Gomez, Y. Dorantes-Gallareta, C. Arce-Salinas, O. Arrieta, Phase II randomized, double-blind, placebo-controlled study of whole-brain irradiation with concomitant chloroquine for brain metastases, Rad. Oncol. 8 (2013) 209-209.

[117] M.R. Rosenfeld, X. Ye, J.G. Supko, S. Desideri, S.A. Grossman, S. Brem, T. Mikkelson, D. Wang, Y.C. Chang, J. Hu, Q. McAfee, J. Fisher, A.B. Troxel, S. Piao, D.F. Heitjan, K.-S. Tan, L. Pontiggia, P.J. O'Dwyer, L.E. Davis, R.K. Amaravadi, A phase I/II trial of hydroxychloroquine in conjunction with radiation therapy and concurrent and adjuvant temozolomide in patients with newly diagnosed glioblastoma multiforme, Autophagy 10(8) (2014) 1359-1368.

[118] M. Bolla , D. Gonzalez , P. Warde , J.B. Dubois , R.-O. Mirimanoff , G. Storme , J. Bernier , A. Kuten , C. Sternberg, T. Gil , L. Collette , M. Pierart Improved Survival in Patients with Locally Advanced Prostate Cancer Treated with Radiotherapy and Goserelin, New Engl. J. Med. 337(5) (1997) 295-300.

[119] G. Alexander, J. Palmer, M. Tuluc, J. Lin, D.C. Hooper, B. Lu, Immune biomarkers of treatment failure for a patient with renal cell carcinoma on a Phase I trial of pembrolizumab plus radiotherapy, J. Immunother. Cancer 3(Suppl 2) (2015) P143-P143.

[120] V. Sibaud, I. David, L. Lamant, S. Resseguier, R. Radut, J. Attal, N. Meyer, J.-P. Delord, Acute skin reaction suggestive of pembrolizumab-induced radiosensitization, Melanoma Res. 25(6) (2015) 555-558.

[121] E.D. Kwon, C.G. Drake, H.I. Scher, K. Fizazi, A. Bossi, A.J.M. van den Eertwegh, M. Krainer, N. Houede, R. Santos, H. Mahammedi, S. Ng, M. Maio, F.A. Franke, S. Sundar, N. Agarwal, A.M. Bergman, T.E. Ciuleanu, E. Korbenfeld, L. Sengeløv, S. Hansen, C. Logothetis, T.M. Beer, M.B. McHenry, P. Gagnier, D. Liu, W.R. Gerritsen, C.A.I. for the, Ipilimumab versus 
placebo after radiotherapy in patients with metastatic castration-resistant prostate cancer that had progressed after docetaxel chemotherapy (CA184-043): a multicentre, randomised, double-blind, phase 3 trial, Lancet Oncol. 15(7) (2014) 700-712.

[122] S.F. Slovin, C.S. Higano, O. Hamid, S. Tejwani, A. Harzstark, J.J. Alumkal, H.I. Scher, K. Chin, P. Gagnier, M.B. McHenry, T.M. Beer, Ipilimumab alone or in combination with radiotherapy in metastatic castration-resistant prostate cancer: results from an open-label, multicenter phase I/II study, Ann. Oncol. 24(7) (2013) 1813-1821.

[123] M.J. Edelman, W. Burrows, M.J. Krasna, M. Bedor, R. Smith, M. Suntharalingam, Phase I trial of carboplatin/paclitaxel/bortezomib and concurrent radiotherapy followed by surgical resection in Stage III non-small cell lung cancer, Lung Cancer 68(1) (2010) 84-88.

[124] G.J. Kubicek, R.S. Axelrod, M. Machtay, P.H. Ahn, P.R. Anne, S. Fogh, D. Cognetti, T.J. Myers, W.J. Curran Jr, A.P. Dicker, Phase I Trial Using the Proteasome Inhibitor Bortezomib and Concurrent Chemoradiotherapy for Head-and-Neck Malignancies, Int. J. Rad. Oncol. Biol. Phys. 83(4) (2012) 1192-1197.

[125] G.J. Kubicek, M. Werner-Wasik, M. Machtay, G. Mallon, T. Myers, M. Ramirez, D. Andrews, W.J. Curran, A.P. Dicker, A phase I trial using the proteasome inhibitor bortezomib and concurrent temozolomide and radiotherapy for CNS malignancies, Int. J. Rad. Oncol. Biol. Phys. 74(2) (2009) 433-439.

[126] Y. Zhao, N.R. Foster, J.P. Meyers, S.P. Thomas, D.W. Northfelt, K.M. Rowland Jr, B.I. Mattar, D.B. Johnson, J.R. Molina, S.J. Mandrekar, S.E. Schild, J.D. Bearden Iii, M.-C. Aubry, A.A. Adjei, A Phase I/II Study of Bortezomib in Combination with Paclitaxel, Carboplatin, and Concurrent Thoracic Radiation Therapy for Non-Small-Cell Lung Cancer: North Central Cancer Treatment Group (NCCTG)-N0321, J. Thorac. Oncol. 10(1) (2015) 172-180.

[127] S. Dische, Chemical sensitizers for hypoxic cells: a decade of experience in clinical radiotherapy, Radiother. Oncol 3(2) (1985) 97-115.

[128] S.M. Elbashir, J. Harborth, W. Lendeckel, A. Yalcin, K. Weber, T. Tuschl, Duplexes of 21nucleotide RNAs mediate RNA interference in cultured mammalian cells, Nature 411(6836) (2001) 494-498.

[129] D.W. Bartlett, M.E. Davis, Insights into the kinetics of siRNA-mediated gene silencing from live-cell and live-animal bioluminescent imaging, Nucleic Acids Res. 34(1) (2006) 322333.

[130] G.S. Higgins, R. Prevo, Y.-F. Lee, T. Helleday, R.J. Muschel, S. Taylor, M. Yoshimura, I.D. Hickson, E.J. Bernhard, W.G. McKenna, A Small Interfering RNA Screen of Genes Involved in DNA Repair Identifies Tumor-Specific Radiosensitization by POLQ Knockdown, Cancer Res. 70(7) (2010) 2984-2993.

[131] D.W. Abbott, J.T. Holt, M.L. Freeman, Double-Strand Break Repair Deficiency and Radiation Sensitivity in BRCA2 Mutant Cancer Cells, J. Natl. Cancer Inst. 90(13) (1998) 978985.

[132] G. Zhou, X. Cheng, S. Wu, X. Jiang, X. Shi, J. Chen, J. Zhang, J. Zhao, Preparation and antitumor activity of a polymeric derivative of methotrexate, Am. J. Med. Sci. 344(4) (2012) 294-299.

[133] Y. Nimura, T. Kawata, K. Uzawa, J. Okamura, C. Liu, M. Saito, H. Shimada, N. Seki, A. Nakagawara, H. Ito, T. Ochiai, H. Tanzawa, Silencing Ku80 using small interfering RNA enhanced radiation sensitivity in vitro and in vivo, Int. J. Oncol. 30(6) (2007) 1477-1484.

[134] Z. Khan, A.A. Khan, G.B.K.S. Prasad, N. Khan, R.P. Tiwari, P.S. Bisen, Growth inhibition and chemo-radiosensitization of head and neck squamous cell carcinoma (HNSCC) by survivinsiRNA lentivirus, Radiother. Oncol. 118(2) (2016) 359-368. 
[135] B. Keck, S. Wach, H. Taubert, S. Zeiler, O.J. Ott, F. Kunath, A. Hartmann, S. Bertz, C. Weiss, P. Hönscheid, S. Schellenburg, C. Rödel, G.B. Baretton, R. Sauer, R. Fietkau, B. Wullich, F.S. Krause, K. Datta, M.H. Muders, Neuropilin-2 and its ligand VEGF-C predict treatment response after transurethral resection and radiochemotherapy in bladder cancer patients, Int. J. Cancer 136(2) (2015) 443-451.

[136] M.H. Muders, H. Zhang, E. Wang, D.J. Tindall, K. Datta, Vascular Endothelial Growth Factor-C Protects Prostate Cancer Cells from Oxidative Stress by the Activation of Mammalian Target of Rapamycin Complex-2 and AKT-1, Cancer Res. 69(15) (2009) 6042-6048.

[137] F. Bruzzese, M. Rocco, S. Castelli, E. Di Gennaro, A. Desideri, A. Budillon, Synergistic antitumor effect between vorinostat and topotecan in small cell lung cancer cells is mediated by generation of reactive oxygen species and DNA damage-induced apoptosis, Mol. Cancer Ther. 8 (2009).

[138] N. Domanitskaya, J. Wangari-Talbot, J. Jacobs, E. Peiffer, Y. Mahdaviyeh, C. Paulose, E. Malofeeva, K. Foster, K.Q. Cai, Y. Zhou, B. Egleston, E. Hopper-Borge, Abcc10 status affects mammary tumour growth, metastasis, and docetaxel treatment response, Br. J. Cancer 111(4) (2014) 696-707.

[139] S. Grosso, J. Doyen, S.K. Parks, T. Bertero, A. Paye, B. Cardinaud, P. Gounon, S. LacasGervais, A. Noel, J. Pouyssegur, P. Barbry, N.M. Mazure, B. Mari, MiR-210 promotes a hypoxic phenotype and increases radioresistance in human lung cancer cell lines, Cell Death Dis. 4 (2013) e544.

[140] P. Angart, D. Vocelle, C. Chan, S.P. Walton, Design of siRNA Therapeutics from the Molecular Scale, Pharmaceuticals 6(4) (2013) 440-468.

[141] M. Foldvari, D.W. Chen, N. Nafissi, D. Calderon, L. Narsineni, A. Rafiee, Non-viral gene therapy: Gains and challenges of non-invasive administration methods, J. Control. Rel.

[142] A.L. Jackson, P.S. Linsley, Recognizing and avoiding siRNA off-target effects for target identification and therapeutic application, Nat. Rev. Drug Discov. 9(1) (2010) 57-67.

[143] J.H. Jeong, H. Mok, Y.-K. Oh, T.G. Park, siRNA Conjugate Delivery Systems, Bioconjugate Chem. 20(1) (2009) 5-14.

[144] E. Miele, G.P. Spinelli, E. Miele, F.E. Di, E. Ferretti, S. Tomao, A. Gulino, Nanoparticlebased delivery of small interfering RNA: challenges for cancer therapy, Int. J. Nanomed. 7 (2012) 3637-3657.

[145] T. Sugo, M. Terada, T. Oikawa, K. Miyata, S. Nishimura, E. Kenjo, M. OgasawaraShimizu, Y. Makita, S. Imaichi, S. Murata, K. Otake, K. Kikuchi, M. Teratani, Y. Masuda, T. Kamei, S. Takagahara, S. Ikeda, T. Ohtaki, H. Matsumoto, Development of antibody-siRNA conjugate targeted to cardiac and skeletal muscles, Journal of Controlled Release 237 (2016) 113.

[146] A. Wittrup, J. Lieberman, Knocking down disease: a progress report on siRNA therapeutics, Nat. Rev. Genet. 16(9) (2015) 543-552.

[147] P. del Pino, A. Munoz-Javier, D. Vlaskou, P. Rivera Gil, C. Plank, W.J. Parak, Gene Silencing Mediated by Magnetic Lipospheres Tagged with Small Interfering RNA, Nano Lett. 10(10) (2010) 3914-3921.

[148] C. Ganas, A. Weiß, M. Nazarenus, S. Rösler, T. Kissel, P. Rivera_Gil, W.J. Parak, Biodegradable capsules as non-viral vectors for in vitro delivery of PEI/siRNA polyplexes for efficient gene silencing, J. Control. Rel. 196 (2014) 132-138.

[149] J. Conde, A. Ambrosone, Y. Hernandez, F. Tian, M. McCully, C.C. Berry, P.V. Baptista, C. Tortiglione, J.M. de la Fuente, 15 years on siRNA delivery: Beyond the State-of-the-Art on inorganic nanoparticles for RNAi therapeutics, Nano Today 10(4) (2015) 421-450. 
[150] H.-I. Chang, M.-K. Yeh, Clinical development of liposome-based drugs: formulation, characterization, and therapeutic efficacy, Int. J. Nanomed. 7 (2012) 49-60.

[151] B.S. Pattni, V.V. Chupin, V.P. Torchilin, New Developments in Liposomal Drug Delivery, Chem. Rev. 115(19) (2015) 10938-10966.

[152] A. Masotti, G. Mossa, C. Cametti, G. Ortaggi, A. Bianco, N.D. Grosso, D. Malizia, C. Esposito, Comparison of different commercially available cationic liposome-DNA lipoplexes: Parameters influencing toxicity and transfection efficiency, Coll. Surf. B: Biointerf. 68(2) (2009) 136-144.

[153] D. Zhi, S. Zhang, S. Cui, Y. Zhao, Y. Wang, D. Zhao, The Headgroup Evolution of Cationic Lipids for Gene Delivery, Bioconjugate Chem. 24(4) (2013) 487-519.

[154] S. Akhtar, I. Benter, Toxicogenomics of non-viral drug delivery systems for RNAi: Potential impact on siRNA-mediated gene silencing activity and specificity, Adv. Drug Deliv. Rev. 59(2-3) (2007) 164-182.

[155] G.R. Dakwar, K. Braeckmans, J. Demeester, W. Ceelen, S.C.D. Smedt, K. Remaut, Disregarded Effect of Biological Fluids in siRNA Delivery: Human Ascites Fluid Severely Restricts Cellular Uptake of Nanoparticles, ACS Appl. Mater. \& Interf. 7(43) (2015) 2432224329.

[156] K.B. Knudsen, H. Northeved, P. Kumar Ek, A. Permin, T. Gjetting, T.L. Andresen, S. Larsen, K.M. Wegener, J. Lykkesfeldt, K. Jantzen, S. Loft, P. Møller, M. Roursgaard, In vivo toxicity of cationic micelles and liposomes, Nanomed.: Nanotechnol. Biol. Med. 11(2) (2015) 467-477.

[157] K. Remaut, B. Lucas, K. Braeckmans, N.N. Sanders, J. Demeester, S.C. De Smedt, Protection of oligonucleotides against nucleases by pegylated and non-pegylated liposomes as studied by fluorescence correlation spectroscopy, J. Control. Rel. 110(1) (2005) 212-226.

[158] L.C. Gomes-da-Silva, N.A. Fonseca, V. Moura, M.C. Pedroso de Lima, S. Simões, J.N. Moreira, Lipid-Based Nanoparticles for siRNA Delivery in Cancer Therapy: Paradigms and Challenges, Acc. Chem. Res. 45(7) (2012) 1163-1171.

[159] T. Gjetting, N.S. Arildsen, C.L. Christensen, T.T. Poulsen, J.A. Roth, V.N. Handlos, H.S. Poulsen, In vitro and in vivo effects of polyethylene glycol (PEG)-modified lipid in DOTAP/cholesterol-mediated gene transfection, Int. J. Nanomed. 5 (2010) 371-383.

[160] M.B. de Jesus, I.S. Zuhorn, Solid lipid nanoparticles as nucleic acid delivery system: Properties and molecular mechanisms, Journal of Controlled Release 201 (2015) 1-13.

[161] S.C. Semple, A. Akinc, J. Chen, A.P. Sandhu, B.L. Mui, C.K. Cho, D.W.Y. Sah, D. Stebbing, E.J. Crosley, E. Yaworski, I.M. Hafez, J.R. Dorkin, J. Qin, K. Lam, K.G. Rajeev, K.F. Wong, L.B. Jeffs, L. Nechev, M.L. Eisenhardt, M. Jayaraman, M. Kazem, M.A. Maier, M. Srinivasulu, M.J. Weinstein, Q. Chen, R. Alvarez, S.A. Barros, S. De, S.K. Klimuk, T. Borland, V. Kosovrasti, W.L. Cantley, Y.K. Tam, M. Manoharan, M.A. Ciufolini, M.A. Tracy, A. de Fougerolles, I. MacLachlan, P.R. Cullis, T.D. Madden, M.J. Hope, Rational design of cationic lipids for siRNA delivery, Nat. Biotechnol. 28(2) (2010) 172-176.

[162] J. Tabernero, G.I. Shapiro, P.M. LoRusso, A. Cervantes, G.K. Schwartz, G.J. Weiss, L. Paz-Ares, D.C. Cho, J.R. Infante, M. Alsina, M.M. Gounder, R. Falzone, J. Harrop, A.C.S. White, I. Toudjarska, D. Bumcrot, R.E. Meyers, G. Hinkle, N. Svrzikapa, R.M. Hutabarat, V.A. Clausen, J. Cehelsky, S.V. Nochur, C. Gamba-Vitalo, A.K. Vaishnaw, D.W.Y. Sah, J.A. Gollob, H.A. Burris, First-in-Humans Trial of an RNA Interference Therapeutic Targeting VEGF and KSP in Cancer Patients with Liver Involvement, Cancer Discover. 3(4) (2013) 406-417.

[163] D. Cun, D.K. Jensen, M.J. Maltesen, M. Bunker, P. Whiteside, D. Scurr, C. Foged, H.M. Nielsen, High loading efficiency and sustained release of siRNA encapsulated in PLGA 
nanoparticles: Quality by design optimization and characterization, Eur. J. Pharmaceutics Biophar. 77(1) (2011) 26-35.

[164] U. Lächelt, E. Wagner, Nucleic Acid Therapeutics Using Polyplexes: A Journey of 50 Years (and Beyond), Chem. Rev. 115(19) (2015) 11043-11078.

[165] S. Choosakoonkriang, B.A. Lobo, G.S. Koe, J.G. Koe, C.R. Middaugh, Biophysical Characterization of PEI/DNA Complexes, J. Pharmaceutical Sci. 92(8) (2003) 1710-1722.

[166] J. Suh, H.J. Paik, B.K. Hwang, Ionization of Poly(ethylenimine) and Poly(allylamine) at Various pH's, Bioorg. Chem. 22(3) (1994) 318-327.

[167] J. Rejman, A. Bragonzi, M. Conese, Role of Clathrin- and Caveolae-Mediated Endocytosis in Gene Transfer Mediated by Lipo- and Polyplexes, Mol. Ther. 12(3) (2005) 468-474.

[168] J.-P. Behr, The proton sponge: a trick to enter cells the viruses did not exploit, Chim. 51 (1997) 34-36.

[169] Z.u. Rehman, I.S. Zuhorn, D. Hoekstra, How cationic lipids transfer nucleic acids into cells and across cellular membranes: Recent advances, J. Control. Rel. 166(1) (2013) 46-56.

[170] W.T. Godbey, K.K. Wu, A.G. Mikos, Size matters: molecular weight affects the efficiency of poly(ethylenimine) as a gene delivery vehicle., J. Biomed. Mater. Res. 45(3) (1999) 268-275.

[171] A.C. Richards Grayson, A.M. Doody, D. Putnam, Biophysical and Structural Characterization of Polyethylenimine-Mediated siRNA Delivery in Vitro, Pharmaceutical Res. 23(8) (2006) 1868-1876.

[172] M. Breunig, U. Lungwitz, R. Liebl, C. Fontanari, J. Klar, A. Kurtz, T. Blunk, A. Goepferich, Gene delivery with low molecular weight linear polyethylenimines., J. Gene Med. 7(10) (2005) 1287-1298.

[173] V. Incani, A. Lavasanifar, H. Uludag, Lipid and hydrophobic modification of cationic carriers on route to superior gene vectors, Soft Matt. 6(10) (2010) 2124-2138.

[174] A. Neamnark, O. Suwantong, R.B. K. C, C.Y.M. Hsu, P. Supaphol, H. Uludağ, Aliphatic Lipid Substitution on $2 \mathrm{kDa}$ Polyethylenimine Improves Plasmid Delivery and Transgene Expression, Mol. Pharmaceutics 6(6) (2009) 1798-1815.

[175] M. Jager, S. Schubert, S. Ochrimenko, D. Fischer, U.S. Schubert, Branched and linear poly(ethylene imine)-based conjugates: synthetic modification, characterization, and application, Chem. Soc. Rev. 41(13) (2012) 4755-4767.

[176] A. Malek, O. Merkel, L. Fink, F. Czubayko, T. Kissel, A. Aigner, In vivo pharmacokinetics, tissue distribution and underlying mechanisms of various PEI(-PEG)/siRNA complexes, Toxicol. Appl. Phar. 236(1) (2009) 97-108.

[177] S. Mao, M. Neu, O. Germershaus, O. Merkel, J. Sitterberg, U. Bakowsky, T. Kissel, Influence of Polyethylene Glycol Chain Length on the Physicochemical and Biological Properties of Poly(ethylene imine)-graft-Poly(ethylene glycol) Block Copolymer/SiRNA Polyplexes, Bioconjugate Chem. 17(5) (2006) 1209-1218.

[178] M. Neu, D. Fischer, T. Kissel, Recent advances in rational gene transfer vector design based on poly(ethylene imine) and its derivatives., J. Gene Med. 7(8) (2005) 992-1009.

[179] H. Aldawsari, R. Edrada-Ebel, D.R. Blatchford, R.J. Tate, L. Tetley, C. Dufès, Enhanced gene expression in tumors after intravenous administration of arginine-, lysine- and leucinebearing polypropylenimine polyplex, Biomater. 32(25) (2011) 5889-5899.

[180] G. Creusat, J.-S. Thomann, A. Maglott, B. Pons, M. Dontenwill, E. Guérin, B. Frisch, G. Zuber, Pyridylthiourea-grafted polyethylenimine offers an effective assistance to siRNAmediated gene silencing in vitro and in vivo, J. Control. Rel. 157(3) (2012) 418-426.

[181] G. Creusat, G. Zuber, Self-Assembling Polyethylenimine Derivatives Mediate Efficient siRNA Delivery in Mammalian Cells, ChemBioChem 9(17) (2008) 2787-2789. 
[182] A. Ewe, S. Przybylski, J. Burkhardt, A. Janke, D. Appelhans, A. Aigner, A novel tyrosinemodified low molecular weight polyethylenimine (P10Y) for efficient siRNA delivery in vitro and in vivo, J. Control. Rel. 230 (2016) 13-25.

[183] D. Gutsch, D. Appelhans, S. Höbel, B. Voit, A. Aigner, Biocompatibility and Efficacy of Oligomaltose-Grafted Poly(ethylene imine)s (OM-PEIs) for in Vivo Gene Delivery, Mol. Pharmaceutics 10(12) (2013) 4666-4675.

[184] S. Höbel, A. Loos, D. Appelhans, S. Schwarz, J. Seidel, B. Voit, A. Aigner, Maltose- and maltotriose-modified, hyperbranched poly(ethylene imine)s (OM-PEIs): Physicochemical and biological properties of DNA and siRNA complexes, J. Control. Rel. 149(2) (2011) 146-158.

[185] J. Teo, J.A. McCarroll, C. Boyer, J. Youkhana, S.M. Sagnella, H.T.T. Duong, J. Liu, G. Sharbeen, D. Goldstein, T.P. Davis, M. Kavallaris, P.A. Phillips, A Rationally Optimized Nanoparticle System for the Delivery of RNA Interference Therapeutics into Pancreatic Tumors in Vivo, Biomacromolecules 17(7) (2016) 2337-2351.

[186] B. Schultheis, D. Strumberg, A. Santel, C. Vank, F. Gebhardt, O. Keil, C. Lange, K. Giese, J. Kaufmann, M. Khan, J. Drevs, First-in-Human Phase I Study of the Liposomal RNA Interference Therapeutic Atu027 in Patients With Advanced Solid Tumors, J. Clin. Oncol. (2014).

[187] D. Strumberg, B. Schultheis, U. Traugott, C. Vank, A. Santel, O. Keil, K. Giese, J. Kaufmann, J. Drevs, Phase I clinical development of Atu027, a siRNA formulation targeting PKN3 in patients with advanced solid tumors, Int. J. Clin. Pharmacol. Ther. 50(1) (2012) 76-78.

[188] J.E. Zuckerman, M.E. Davis, Clinical experiences with systemically administered siRNAbased therapeutics in cancer, Nat. Rev. Drug Discov. 14(12) (2015) 843-856.

[189] M.S. Singh, D. Peer, RNA nanomedicines: the next generation drugs?, Curr. Opinion Biotechnol. 39 (2016) 28-34.

[190] M.E. Davis, J.E. Zuckerman, C.H.J. Choi, D. Seligson, A. Tolcher, C.A. Alabi, Y. Yen, J.D. Heidel, A. Ribas, Evidence of RNAi in humans from systemically administered siRNA via targeted nanoparticles, Nature 464(7291) (2010) 1067-1070.

[191] K. Bhattacharya, S.P. Mukherjee, A. Gallud, S.C. Burkert, S. Bistarelli, S. Bellucci, M. Bottini, A. Star, B. Fadeel, Biological interactions of carbon-based nanomaterials: From coronation to degradation, Nanomed.: Nanotechnol. Biol. Med. 12(2) (2016) 333-351.

[192] D. Chimene, D.L. Alge, A.K. Gaharwar, Two-Dimensional Nanomaterials for Biomedical Applications: Emerging Trends and Future Prospects, Adv. Mater. 27(45) (2015) 7261-7284.

[193] S.C. Patel, G. Lalwani, K. Grover, Y.X. Qin, B. Sitharaman, Fabrication and cytocompatibility of in situ crosslinked carbon nanomaterial films, Sci. Rep. 5 (2015).

[194] R.H. Baughman, A.A. Zakhidov, W.A. De Heer, Carbon nanotubes - The route toward applications, Science 297(5582) (2002) 787-792.

[195] A. Bianco, K. Kostarelos, C.D. Partidos, M. Prato, Biomedical applications of functionalised carbon nanotubes, Chem. Commun. (5) (2005) 571-577.

[196] J. Byun, Emerging frontiers of graphene in biomedicine, J. Microbiol. Biotechnol. 25(2) (2015) 145-151.

[197] L. Feng, Z. Liu, Graphene in biomedicine: Opportunities and challenges, Nanomed. 6(2) (2011) 317-324.

[198] L. Lacerda, A. Bianco, M. Prato, K. Kostarelos, Carbon nanotubes as nanomedicines: From toxicology to pharmacology, Adv. Drug Deliv. Rev. 58(14) (2006) 1460-1470.

[199] Y. Zhang, D. Petibone, Y. Xu, M. Mahmood, A. Karmakar, D. Casciano, S. Ali, A.S. Biris, Toxicity and efficacy of carbon nanotubes and graphene: The utility of carbon-based nanoparticles in nanomedicine, Drug Metabolism Rev. 46(2) (2014) 232-246. 
[200] G. Cellot, E. Cilia, S. Cipollone, V. Rancic, A. Sucapane, S. Giordani, L. Gambazzi, H. Markram, M. Grandolfo, D. Scaini, F. Gelain, L. Casalis, M. Prato, M. Giugliano, L. Ballerini, Carbon nanotubes might improve neuronal performance by favouring electrical shortcuts, Nat. Nanotechnol. 4(2) (2009) 126-133.

[201] R.J. Chen, S. Bangsaruntip, K.A. Drouvalakis, N. Wong Shi Kam, M. Shim, Y. Li, W. Kim, P.J. Utz, H. Dai, Noncovalent functionalization of carbon nanotubes for highly specific electronic biosensors, Proc. Natl. Acad. Sci. USA 100(9) (2003) 4984-4989.

[202] Z. Liu, K. Chen, C. Davis, S. Sherlock, Q. Cao, X. Chen, H. Dai, Drug delivery with carbon nanotubes for in vivo cancer treatment, Cancer Res. 68(16) (2008) 6652-6660.

[203] D.T. Mitchell, S.B. Lee, L. Trofin, N. Li, T.K. Nevanen, H. Söderlund, C.R. Martin, Smart nanotubes for bioseparations and biocatalysis, J. Am. Chem. Soc. 124(40) (2002) 11864-11865. [204] S.G. Penn, L. He, M.J. Natan, Nanoparticles for bioanalysis, Curr. Opinion Chem. Biol. 7(5) (2003) 609-615.

[205] G. Cirillo, S. Hampel, U.G. Spizzirri, O.I. Parisi, N. Picci, F. Iemma, Carbon Nanotubes Hybrid Hydrogels in Drug Delivery: A Perspective Review, Biomed. Res. Int. (2014).

[206] P. Chaudhuri, S. Soni, S. Sengupta, Single-walled carbon nanotube-conjugated chemotherapy exhibits increased therapeutic index in melanoma, Nanotechnol. 21(2) (2010).

[207] C. Fabbro, H. Ali-Boucetta, T.D. Ros, K. Kostarelos, A. Bianco, M. Prato, Targeting carbon nanotubes against cancer, Chem. Commun. 48(33) (2012) 3911-3926.

[208] Z. Liu, A.C. Fan, K. Rakhra, S. Sherlock, A. Goodwin, X. Chen, Q. Yang, D.W. Felsher, H. Dai, Supramolecular stacking of doxorubicin on carbon nanotubes for in vivo cancer therapy, Angew. Chem. Int. Ed. 48(41) (2009) 7668-7672.

[209] M.I. Sajid, U. Jamshaid, T. Jamshaid, N. Zafar, H. Fessi, A. Elaissari, Carbon nanotubes from synthesis to in vivo biomedical applications, Int. J. Pharmaceutics 501(1-2) (2016) 278-299. [210] C. Samorì, H. Ali-Boucetta, R. Sainz, C. Guo, F.M. Toma, C. Fabbro, T. Da Ros, M. Prato, K. Kostarelos, A. Bianco, Enhanced anticancer activity of multi-walled carbon nanotubemethotrexate conjugates using cleavable linkers, Chem. Commun. 46(9) (2010) 1494-1496.

[211] H. Wang, J. Wang, X. Deng, H. Sun, Z. Shi, Z. Gu, Y. Liu, Y. Zhao, Biodistribution of carbon single-wall carbon nanotubes in mice, J. Nanosci. Nanotechnol. 4(8) (2004) 1019-1024.

[212] P. Wick, P. Manser, L.K. Limbach, U. Dettlaff-Weglikowska, F. Krumeich, S. Roth, W.J. Stark, A. Bruinink, The degree and kind of agglomeration affect carbon nanotube cytotoxicity, Toxicol. Lett. 168(2) (2007) 121-131.

[213] C.W. Lam, J.T. James, R. McCluskey, S. Arepalli, R.L. Hunter, A review of carbon nanotube toxicity and assessment of potential occupational and environmental health risks, Crit. Rev. Toxicol. 36(3) (2006) 189-217.

[214] C.W. Lam, J.T. James, R. McCluskey, R.L. Hunter, Pulmonary toxicity of single-wall carbon nanotubes in mice 7 and 90 days after intractracheal instillation, Toxicol. Sci. 77(1) (2004) 126-134.

[215] B. Sitharaman, X. Shi, X.F. Walboomers, H. Liao, V. Cuijpers, L.J. Wilson, A.G. Mikos, J.A. Jansen, In vivo biocompatibility of ultra-short single-walled carbon nanotube/biodegradable polymer nanocomposites for bone tissue engineering, Bone 43(2) (2008) 362-370.

[216] D. Van Berlo, V. Wilhelmi, A.W. Boots, M. Hullmann, T.A.J. Kuhlbusch, A. Bast, R.P.F. Schins, C. Albrecht, Apoptotic, inflammatory, and fibrogenic effects of two different types of multi-walled carbon nanotubes in mouse lung, Archives Toxicol. 88(9) (2014) 1725-1737.

[217] H. Nagai, Y. Okazaki, S.H. Chew, N. Misawa, Y. Yamashita, S. Akatsuka, T. Ishihara, K. Yamashita, Y. Yoshikawa, H. Yasui, L. Jiang, H. Ohara, T. Takahashi, G. Ichihara, K. Kostarelos, Y. Miyata, H. Shinohara, S. Toyokuni, Diameter and rigidity of multiwalled carbon 
nanotubes are critical factors in mesothelial injury and carcinogenesis, Proc. Natl. Acad. Sci. USA 108(49) (2011).

[218] C.A. Poland, R. Duffin, I. Kinloch, A. Maynard, W.A.H. Wallace, A. Seaton, V. Stone, S. Brown, W. MacNee, K. Donaldson, Carbon nanotubes introduced into the abdominal cavity of mice show asbestos-like pathogenicity in a pilot study, Nat. Nanotechnol. 3(7) (2008) 423-428.

[219] M. Zhang, T. Yamaguchi, S. Iijima, M. Yudasaka, Size-dependent biodistribution of carbon nanohorns in vivo, Nanomed.: Nanotechnol. Biol. Med. 9(5) (2013) 657-664.

[220] S. Benedetti, B. Nuvoli, S. Catalani, R. Galati, Reactive oxygen species a double-edged sword for mesothelioma, Oncotarget 6(19) (2015) 16848-16865.

[221] D. Tasis, N. Tagmatarchis, A. Bianco, M. Prato, Chemistry of carbon nanotubes, Chem. Rev. 106(3) (2006) 1105-1136.

[222] G. Pastorin, Crucial functionalizations of carbon nanotubes for improved drug delivery: A valuable option?, Pharmaceutical Res. 26(4) (2009) 746-769.

[223] P. Singh, S. Campidelli, S. Giordani, D. Bonifazi, A. Bianco, M. Prato, Organic functionalisation and characterisation of single-walled carbon nanotubes, Chem. Soc. Rev. 38(8) (2009) 2214-2230.

[224] C. Wang, Z.X. Guo, S. Fu, W. Wu, D. Zhu, Polymers containing fullerene or carbon nanotube structures, Prog. Polym. Sci. 29(11) (2004) 1079-1141.

[225] J. Yang, W. Li, Q. Li, S. Wu, B. Yu, X. Jing, Oxygen adsorption by carbon nanotubes and its application in radiotherapy, IET Nanobiotechnol. 1(1) (2007) 10-14.

[226] A.H. Castro Neto, F. Guinea, N.M.R. Peres, K.S. Novoselov, A.K. Geim, The electronic properties of graphene, Rev. Modern Phys. 81(1) (2009) 109-162.

[227] K.S. Novoselov, A.K. Geim, S.V. Morozov, D. Jiang, Y. Zhang, S.V. Dubonos, I.V. Grigorieva, A.A. Firsov, Electric field in atomically thin carbon films, Science 306(5696) (2004) 666-669.

[228] C.N.R. Rao, A.K. Sood, K.S. Subrahmanyam, A. Govindaraj, Graphene: The new twodimensional nanomaterial, Angew. Chem. Int. Ed. 48(42) (2009) 7752-7777.

[229] Y. Wang, Z. Li, J. Wang, J. Li, Y. Lin, Graphene and graphene oxide: Biofunctionalization and applications in biotechnology, Trends in Biotechnol. 29(5) (2011) 205-212.

[230] J. Huang, C. Zong, H. Shen, M. Liu, B. Chen, B. Ren, Z. Zhang, Mechanism of Cellular Uptake of Graphene Oxide Studied by Surface-Enhanced Raman Spectroscopy, Small 8(16) (2012) 2577-2584.

[231] S. Makharza, G. Cirillo, A. Bachmatiuk, I. Ibrahim, N. Ioannides, B. Trzebicka, S. Hampel, M.H. Ruemmeli, Graphene oxide-based drug delivery vehicles: functionalization, characterization, and cytotoxicity evaluation, J. Nanoparticle Res. 15(12) (2013).

[232] C. Peng, W. Hu, Y. Zhou, C. Fan, Q. Huang, Intracellular imaging with a graphene-based fluorescent probe, Small 6(15) (2010) 1686-1692.

[233] V.C. Sanchez, A. Jachak, R.H. Hurt, A.B. Kane, Biological interactions of graphene-family nanomaterials: An interdisciplinary review, Chem. Res. Toxicol. 25(1) (2012) 15-34.

[234] H. Shen, L. Zhang, M. Liu, Z. Zhang, Biomedical applications of graphene, Theranostics 2(3) (2012) 283-294.

[235] H.Y. Mao, S. Laurent, W. Chen, O. Akhavan, M. Imani, A.A. Ashkarran, M. Mahmoudi, Graphene: Promises, facts, opportunities, and challenges in nanomedicine, Chem. Rev. 113(5) (2013) 3407-3424.

[236] A. Ambrosi, C.K. Chua, B. Khezri, Z. Sofer, R.D. Webster, M. Pumera, Chemically reduced graphene contains inherent metallic impurities present in parent natural and synthetic graphite, Proc. Natl. Acad. Sci. USA 109(32) (2012) 12899-12904. 
[237] A. Bagri, C. Mattevi, M. Acik, Y.J. Chabal, M. Chhowalla, V.B. Shenoy, Structural evolution during the reduction of chemically derived graphene oxide, Nat. Chem. 2(7) (2010) 581-587.

[238] M.A. Creighton, J.R. Rangel-Mendez, J. Huang, A.B. Kane, R.H. Hurt, Graphene-induced adsorptive and optical artifacts during in vitro toxicology assays, Small 9(11) (2013) 1921-1927. [239] W. Hu, C. Peng, M. Lv, X. Li, Y. Zhang, N. Chen, C. Fan, Q. Huang, Protein coronamediated mitigation of cytotoxicity of graphene oxide, ACS nano 5(5) (2011) 3693-3700.

[240] H. Ren, C. Wang, J. Zhang, X. Zhou, D. Xu, J. Zheng, S. Guo, DNA cleavage system of nanosized graphene oxide sheets and copper ions, ACS nano 4(12) (2010) 7169-7174.

[241] M. Wu, R. Kempaiah, P.J.J. Huang, V. Maheshwari, J. Liu, Adsorption and desorption of DNA on graphene oxide studied by fluorescently labeled oligonucleotides, Langmuir 27(6) (2011) 2731-2738.

[242] S.F. Kiew, L.V. Kiew, H.B. Lee, T. Imae, L.Y. Chung, Assessing biocompatibility of graphene oxide-based nanocarriers: A review, J. Control. Rel. 226 (2016) 217-228.

[243] K. Yang, H. Gong, X. Shi, J. Wan, Y. Zhang, Z. Liu, In vivo biodistribution and toxicology of functionalized nano-graphene oxide in mice after oral and intraperitoneal administration, Biomater. 34(11) (2013) 2787-2795.

[244] X. Zhang, J. Yin, C. Peng, W. Hu, Z. Zhu, W. Li, C. Fan, Q. Huang, Distribution and biocompatibility studies of graphene oxide in mice after intravenous administration, Carbon 49(3) (2011) 986-995.

[245] M. Mahmoudi, I. Lynch, M.R. Ejtehadi, M.P. Monopoli, F.B. Bombelli, S. Laurent, Protein-nanoparticle interactions: Opportunities and challenges, Chem. Rev. 111(9) (2011) 56105637.

[246] D. Maiolo, P. Del Pino, P. Metrangolo, W.J. Parak, F. Baldelli Bombelli, Nanomedicine delivery: does protein corona route to the target or off road?, Nanomed. 10(21) (2015) 32313247.

[247] T. Kuila, S. Bose, A.K. Mishra, P. Khanra, N.H. Kim, J.H. Lee, Chemical functionalization of graphene and its applications, Prog. Mater. Sci. 57(7) (2012) 1061-1105.

[248] S. Makharza, G. Cirillo, A. Bachmatiuk, O. Vittorio, R.G. Mendes, S. Oswald, S. Hampel, M.H. Ruemmeli, Size-dependent nanographene oxide as a platform for efficient carboplatin release, J. Mater. Chem. B 1(44) (2013) 6107-6114.

[249] S. Makharza, O. Vittorio, G. Cirillo, S. Oswald, E. Hinde, M. Kavallaris, B. Buechner, M. Mertig, S. Hampel, Graphene Oxide - Gelatin Nanohybrids as Functional Tools for Enhanced Carboplatin Activity in Neuroblastoma Cells, Pharmaceutical Res. 32(6) (2015) 2132-2143.

[250] J. Drbohlavova, J. Chomoucka, V. Adam, M. Ryvolova, T. Eckschlager, J. Hubalek, R. Kizek, Nanocarriers for anticancer drugs--new trends in nanomedicine, Curr. Drug Metab. 14(5) (2013) 547-64.

[251] V. Rastogi, P. Yadav, S.S. Bhattacharya, A.K. Mishra, N. Verma, A. Verma, J.K. Pandit, Carbon nanotubes: an emerging drug carrier for targeting cancer cells, J. Drug Deliv. 2014 (2014) 670815.

[252] A. Mignot, C. Truillet, F. Lux, L. Sancey, C. Louis, F. Denat, F. Boschetti, L. Bocher, A. Gloter, O. Stephan, R. Antoine, P. Dugourd, D. Luneau, G. Novitchi, L.C. Figueiredo, P.C. de Morais, L. Bonneviot, B. Albela, F. Ribot, L. Van Lokeren, I. Dechamps-Olivier, F. Chuburu, G. Lemercier, C. Villiers, P.N. Marche, G. Le Duc, S. Roux, O. Tillement, P. Perriat, A top-down synthesis route to ultrasmall multifunctional Gd-based silica nanoparticles for theranostic applications, Chem. 19(19) (2013) 6122-36. 
[253] H. Maeda, The enhanced permeability and retention (EPR) effect in tumor vasculature: the key role of tumor-selective macromolecular drug targeting, Adv. Enzyme Regul. 41 (2001) 189207.

[254] H. Maeda, G.Y. Bharate, J. Daruwalla, Polymeric drugs for efficient tumor-targeted drug delivery based on EPR-effect, European journal of pharmaceutics and biopharmaceutics : official journal of Arbeitsgemeinschaft fur Pharmazeutische Verfahrenstechnik e.V 71(3) (2009) 409-19.

[255] T.M. Allen, F.J. Martin, Advantages of liposomal delivery systems for anthracyclines, Seminars in oncology 31(6 Suppl 13) (2004) 5-15.

[256] F. Danhier, O. Feron, V. Preat, To exploit the tumor microenvironment: Passive and active tumor targeting of nanocarriers for anti-cancer drug delivery, J. Control. Rel. 148(2) (2010) 13546.

[257] L. Lacerda, J. Russier, G. Pastorin, M.A. Herrero, E. Venturelli, H. Dumortier, K.T. AlJamal, M. Prato, K. Kostarelos, A. Bianco, Translocation mechanisms of chemically functionalised carbon nanotubes across plasma membranes, Biomater. 33(11) (2012) 3334-43.

[258] H. Maeda, J. Wu, T. Sawa, Y. Matsumura, K. Hori, Tumor vascular permeability and the EPR effect in macromolecular therapeutics: a review, J. Control. Rel. 65(1-2) (2000) 271-84.

[259] B.S. Wong, S.L. Yoong, A. Jagusiak, T. Panczyk, H.K. Ho, W.H. Ang, G. Pastorin, Carbon nanotubes for delivery of small molecule drugs, Adv. Drug Deliv. Rev. 65(15) (2013) 1964-2015. [260] D.W. Northfelt, F.J. Martin, P. Working, P.A. Volberding, J. Russell, M. Newman, M.A. Amantea, L.D. Kaplan, Doxorubicin encapsulated in liposomes containing surface-bound polyethylene glycol: pharmacokinetics, tumor localization, and safety in patients with AIDSrelated Kaposi's sarcoma, J. Clin. Pharmacol. 36(1) (1996) 55-63.

[261] W.L. Lu, X.R. Qi, Q. Zhang, R.Y. Li, G.L. Wang, R.J. Zhang, S.L. Wei, A pegylated liposomal platform: pharmacokinetics, pharmacodynamics, and toxicity in mice using doxorubicin as a model drug, J. Pharmacol. Sci. 95(3) (2004) 381-9.

[262] N. Yamaguchi, T. Fujii, S. Aoi, P.S. Kozuch, G.N. Hortobagyi, R.H. Blum, Comparison of cardiac events associated with liposomal doxorubicin, epirubicin and doxorubicin in breast cancer: a Bayesian network meta-analysis, Eur. J. Cancer 51(16) (2015) 2314-20.

[263] H.A. Blair, E.D. Deeks, Albumin-Bound Paclitaxel: A Review in Non-Small Cell Lung Cancer, Drugs 75(17) (2015) 2017-24.

[264] J.W. Nichols, Y.H. Bae, EPR: Evidence and fallacy, J. Control. Rel. 190 (2014) 451-64.

[265] T. Dai, N. Li, F. Han, H. Zhang, Y. Zhang, Q. Liu, AMP-guided tumour-specific nanoparticle delivery via adenosine A receptor, Biomater. 83 (2016) 37-50.

[266] A. Popovtzer, A. Mizrachi, M. Motiei, D. Bragilovski, L. Lubimov, M. Levi, O. Hilly, I. Ben-Aharon, R. Popovtzer, Actively targeted gold nanoparticles as novel radiosensitizer agents: an in vivo head and neck cancer model, Nanoscale (2016).

[267] V.H. Shargh, H. Hondermarck, M. Liang, Antibody-targeted biodegradable nanoparticles for cancer therapy, Nanomed. 11(1) (2016) 63-79.

[268] M.K. Yu, J. Park, S. Jon, Targeting strategies for multifunctional nanoparticles in cancer imaging and therapy, Theranostics 2(1) (2012) 3-44.

[269] R. Duncan, The dawning era of polymer therapeutics, Nat. Rev. Drug Discov. 2(5) (2003) 347-360.

[270] F. Greco, M.J. Vicent, Combination therapy: Opportunities and challenges for polymerdrug conjugates as anticancer nanomedicines, Adv. Drug Deliv. Rev. 61(13) (2009) 1203-1213.

[271] R. Haag, F. Kratz, Polymer therapeutics: Concepts and applications, Angew. Chem. Int. Ed. 45(8) (2006) 1198-1215. 
[272] G. Cirillo, M. Curcio, O. Vittorio, F. Iemma, D. Restuccia, U.G. Spizzirri, F. Puoci, N. Picci, Polyphenol Conjugates and Human Health: A Perspective Review, Crit. Rev. Food Sci. Nutr. 56(2) (2016) 326-337.

[273] E. Pérez-Herrero, A. Fernández-Medarde, Advanced targeted therapies in cancer: Drug nanocarriers, the future of chemotherapy, Eur. J. Pharm. Biopharm. 93 (2015) 52-79.

[274] R. Gaspar, R. Duncan, Polymeric carriers: Preclinical safety and the regulatory implications for design and development of polymer therapeutics, Adv. Drug Deliv. Rev. 61(13) (2009) 1220-1231.

[275] S.A. Low, J. Kopeček, Targeting polymer therapeutics to bone, Adv. Drug Deliv. Rev. 64(12) (2012) 1189-1204.

[276] A.K.R. Lytton-Jean, K.J. Kauffman, J.C. Kaczmarek, R. Langer, Cancer nanotherapeutics in clinical trials, Cancer Treatment Res., 2015, pp. 293-322.

[277] S. Abe, M. Otsuki, Styrene maleic acid neocarzinostatin treatment for hepatocellular carcinoma, Curr. Med. Chem. - Anti-Cancer Agents 2(6) (2002) 715-726.

[278] P.A. Dinndorf, J. Gootenberg, M.H. Cohen, P. Keegan, R. Pazdur, FDA drug approval summary: Pegaspargase (Oncaspar®) for the first-line treatment of children with acute lymphoblastic leukemia (ALL), Oncol. 12(8) (2007) 991-998.

[279] R. Masetti, A. Pession, First-line treatment of acute lymphoblastic leukemia with pegasparaginase, Biologics: Targets and Therapy 3 (2009) 359-368.

[280] T. Taguchi, T. Saito, J. Ota, I. Nakao, K. Ohashi, H. Nakamura, T. Konno, Phase II study of YM 881 (zinostatin stimalamer) suspension injected into the hepatic artery, Jpn. J. Cancer Chemother. 18(10) (1991) 1665-1675.

[281] M. Amzerin, M. Mokrim, H. Errihani, M.J. Piccart, Iterative and prolonged remission in metastatic breast cancer using pegylated irinotecan: A case report, J. Med. Case Rep. 9(1) (2015). [282] V.R. Caiolfa, M. Zamai, A. Fiorino, E. Frigerio, C. Pellizzoni, R. D'Argy, A. Ghiglieri, M.G. Castelli, M. Farao, E. Pesenti, M. Gigli, F. Angelucci, A. Suarato, Polymer-bound camptothecin: Initial biodistribution and antitumour activity studies, J. Control. Rel. 65(1-2) (2000) 105-119.

[283] T. Lammers, Improving the efficacy of combined modality anticancer therapy using HPMA copolymer-based nanomedicine formulations, Adv. Drug Deliv. Rev. 62(2) (2010) 203230.

[284] E. López-Miranda, J. Cortés, Etirinotecan pegol for the treatment of breast cancer, Expert Opinion Pharmacother. 17(5) (2016) 727-734.

[285] J.M. Meerum Terwogt, W.W. Ten Bokkel Huinink, J.H.M. Schellens, M. Schot, I.A.M. Mandjes, M.G. Zurlo, M. Rocchetti, H. Rosing, F.J. Koopman, J.H. Beljnen, Phase I clinical and pharmacokinetic study of PNU166945, a novel water-soluble polymer-conjugated prodrug of paclitaxel, Anti-Cancer Drugs 12(4) (2001) 315-323.

[286] D.P. Nowotnik, E. Cvitkovic, ProLindac ${ }^{\text {TM }}$ (AP5346): A review of the development of an HPMA DACH platinum Polymer Therapeutic, Adv. Drug Deliv. Rev. 61(13) (2009) 1214-1219. [287] M.E.R. O'Brien, M.A. Socinski, A.Y. Popovich, I.N. Bondarenko, A. Tomova, B.T. Bilynsky, Y.S. Hotko, V.L. Ganul, I.Y. Kostinsky, A.J. Eisenfeld, L. Sandalic, F.B. Oldham, B. Bandstra, A.B. Sandler, J.W. Singer, Randomized phase III trial comparing single-agent paclitaxel poliglumex (CT-2103, PPX) with single-agent gemcitabine or vinorelbine for the treatment of PS 2 patients with chemotherapy-naïve advanced non-small cell lung cancer, J. Thorac. Oncol. 3(7) (2008) 728-734.

[288] J.M. Rademaker-Lakhai, C. Terret, S.B. Howell, C.M. Baud, R.F. De Boer, D. Pluim, J.H. Beijnen, J.H.M. Schellens, J.P. Droz, A phase I and pharmacological study of the platinum 
polymer AP5280 given as an intravenous infusion once every 3 weeks in patients with solid tumors, Clin. Cancer Res. 10(10) (2004) 3386-3395.

[289] K. Riebeseel, E. Biedermann, R. Löser, N. Breiter, R. Hanselmann, R. Mülhaupt, C. Unger, F. Kratz, Polyethylene glycol conjugates of methotrexate varying in their molecular weight from MW 750 to MW 40000: Synthesis, characterization, and structure-activity relationships in vitro and in vivo, Bioconjugate Chem. 13(4) (2002) 773-785.

[290] L.W. Seymour, D.R. Ferry, D.J. Kerr, D. Rea, M. Whitlock, R. Poyner, C. Boivin, S. Hesselewood, C. Twelves, R. Blackie, A. Schatzlein, D. Jodrell, D. Bissett, H. Calvert, M. Lind, A. Robbins, S. Burtles, R. Duncan, J. Cassidy, Phase II studies of polymer-doxorubicin (PK1, FCE28068) in the treatment of breast, lung and colorectal cancer, Int. J. Oncol. 34(6) (2009) 1629-1636.

[291] R. Sharma, N. Singla, S. Mehta, T. Gaba, R.K. Rawal, H.S. Rao, T.R. Bhardwaj, Recent advances in polymer drug conjugates, Mini-Rev. Med. Chem. 15(9) (2015) 751-761.

[292] J.W. Singer, R. Bhatt, J. Tulinsky, K.R. Buhler, E. Heasley, P. Klein, P. De Vries, Watersoluble poly-(L-glutamic acid)-Gly-camptothecin conjugates enhance camptothecin stability and efficacy in vivo, J. Control. Rel. 74(1-3) (2001) 243-247.

[293] M. Vandana, S.K. Sahoo, Long circulation and cytotoxicity of PEGylated gemcitabine and its potential for the treatment of pancreatic cancer, Biomater. 31(35) (2010) 9340-9356.

[294] H. Xiao, R. Qi, S. Liu, X. Hu, T. Duan, Y. Zheng, Y. Huang, X. Jing, Biodegradable polymer - cisplatin(IV) conjugate as a pro-drug of cisplatin(II), Biomater. 32(30) (2011) 77327739.

[295] X. Zhang, Y. Li, X. Chen, X. Wang, X. Xu, Q. Liang, J. Hu, X. Jing, Synthesis and characterization of the paclitaxel/MPEG-PLA block copolymer conjugate, Biomater. 26(14) (2005) 2121-2128.

[296] C. Li, S. Wallace, Polymer-drug conjugates: Recent development in clinical oncology, Adv. Drug Deliv. Rev. 60(8) (2008) 886-898.

[297] H. Ringsdorf, STRUCTURE AND PROPERTIES OF PHARMACOLOGICALLY ACTIVE POLYMERS, J. Polym. Sci. Polym. Symp. (51) (1975) 135-153.

[298] T.M. Allen, Ligand-targeted therapeutics in anticancer therapy, Nat. Rev. Cancer 2(10) (2002) 750-763.

[299] M. Sobczak, C. Debek, E. Oledzka, R. Kozłowski, Polymeric systems of antimicrobial peptides-strategies and potential applications, Molecules 18(11) (2013) 14122-14137.

[300] R. Duncan, Polymer conjugates as anticancer nanomedicines, Nat. Rev. Cancer 6(9) (2006) 688-701.

[301] H. Maeda, Macromolecular therapeutics in cancer treatment: The EPR effect and beyond, J. Control. Rel. 164(2) (2012) 138-144.

[302] Y. Luo, G.D. Prestwich, Cancer-targeted polymeric drugs, Curr. Cancer Drug Targets 2(3) (2002) 209-226.

[303] N. Goodarzi, R. Varshochian, G. Kamalinia, F. Atyabi, R. Dinarvand, A review of polysaccharide cytotoxic drug conjugates for cancer therapy, Carbohydrate Polymers 92(2) (2013) 1280-1293.

[304] R. Satchi-Fainaro, R. Duncan, C.M. Barnes, Polymer therapeutics for cancer: Current status and future challenges, Adv. Polym. Sci., 2006, pp. 1-65.

[305] D. Nevozhay, R. Budzynska, U. Kanska, M. Jagiello, M.S. Omar, J. Boratynski, A. Opolski, Antitumor properties and toxicity of dextran-methotrexate conjugates are dependent on the molecular weight of the carrier, Anticancer Res 26(2 A) (2006) 1135-1143. 
[306] P.A. Vasey, S.B. Kaye, R. Morrison, C. Twelves, P. Wilson, R. Duncan, A.H. Thomson, L.S. Murray, T.E. Hilditch, T. Murray, S. Burtles, D. Fraier, E. Frigerio, J. Cassidy, Phase I clinical and pharmacokinetic study of PK1 [N-(2- hydroxypropyl)methacrylamide copolymer doxorubicin]: First member of a new class of chemotherapeutic agents - Drug-polymer conjugates, Clin. Cancer Res. 5(1) (1999) 83-94.

[307] T. Minko, P. Kopečková, V. Pozharov, J. Kopeček, HPMA copolymer bound adriamycin overcomes MDR1 gene encoded resistance in a human ovarian carcinoma cell line, J. Control. Rel. 54(2) (1998) 223-233.

[308] B. Ř́hová, J. Strohalm, J. Prausová, K. Kubáčková, M. Jelínková, L. Rozprimová, M. Š́rová, D. Plocová, T. Etrych, V. Šubr, T. Mrkvan, M. Kováŕ, K. Ulbrich, Cytostatic and immunomobilizing activities of polymer-bound drugs: Experimental and first clinical data, J. Control. Rel. 91(1-2) (2003) 1-16.

[309] M. Sirova, M. Kabesova, L. Kovar, T. Etrych, J. Strohalm, K. Ulbrich, B. Rihova, HPMA copolymer-bound doxorubicin induces immunogenic tumor cell death, Curr. Med. Chem. 20(38) (2013) 4815-4826.

[310] L.M. Bareford, P.W. Swaan, Endocytic mechanisms for targeted drug delivery, Adv. Drug Deliv. Rev. 59(8) (2007) 748-758.

[311] N. Kamaly, Z. Xiao, P.M. Valencia, A.F. Radovic-Moreno, O.C. Farokhzad, Targeted polymeric therapeutic nanoparticles: Design, development and clinical translation, Chem. Soc. Rev. 41(7) (2012) 2971-3010.

[312] T. Lammers, V. Subr, P. Peschke, R. Kühnlein, W.E. Hennink, K. Ulbrich, F. Kiessling, M. Heilmann, J. Debus, P.E. Huber, G. Storm, Image-guided and passively tumour-targeted polymeric nanomedicines for radiochemotherapy, Br. J. Cancer 99(6) (2008) 900-910.

[313] S. Ke, L. Milas, C. Charnsangavej, S. Wallace, C. Li, Potentiation of radioresponse by polymer-drug conjugates, J. Control. Rel. 74(1-3) (2001) 237-242.

[314] L. Milas, K.A. Mason, N. Hunter, C. Li, S. Wallace, Poly(L-glutamic acid)-paclitaxel conjugate is a potent enhancer of tumor radiocurability, Int. J. Rad. Oncol. Biol. Phys. 55(3) (2003) 707-712.

[315] T. Dipetrillo, L. Milas, D. Evans, P. Akerman, T. Ng, T. Miner, D. Cruff, B. Chauhan, D. Iannitti, D. Harrington, H. Safran, Paclitaxel Poliglumex (PPX-Xyotax) and concurrent radiation for esophageal and gastric cancer: A phase I study, Am. J. Clin. Oncol.: Cancer Clin. Trials 29(4) (2006) 376-379.

[316] N. Wiedenmann, D. Valdecanas, N. Hunter, S. Hyde, T.A. Buchholz, L. Milas, K.A. Mason, 130-nm albumin - bound paclitaxel enhances tumor radiocurability and therapeutic gain, Clin. Cancer Res. 13(6) (2007) 1868-1874.

[317] F. Zhang, E. Lees, F. Amin, P. Rivera_Gil, F. Yang, P. Mulvaney, W.J. Parak, PolymerCoated Nanoparticles: A Universal Tool for Biolabelling Experiments, Small 7(22) (2011) 31133127.

[318] C. He, J. Lu, W. Lin, Hybrid nanoparticles for combination therapy of cancer, J. Control. Rel. 219 (2015) 224-236.

[319] A.C. Nava, M. Cojoc, C. Peitzsch, G. Cirillo, I. Kurth, S. Fuessel, K. Erdmann, D. Kunhardt, O. Vittorio, S. Hampel, A. Dubrovska, Development of novel radiochemotherapy approaches targeting prostate tumor progenitor cells using nanohybrids, Int. J. Cancer 137(10) (2015) 2492-2503.

[320] S. Oliver, O. Vittorio, G. Cirillo, C. Boyer, Enhancing the therapeutic effects of polyphenols with macromolecules, Polymer Chem. 7(8) (2016) 1529-1544. 
[321] U.G. Spizzirri, G. Cirillo, N. Picci, F. Iemma, Recent Development in the Synthesis of EcoFriendly Polymeric Antioxidants, Curr. Org. Chem. 18(23) (2014) 2912-2927.

[322] S. Meucci, M. Travagliati, O. Vittorio, G. Cirillo, L. Masini, V. Voliani, N. Picci, F. Beltram, A. Tredicucci, M. Cecchini, Tubeless biochip for chemical stimulation of cells in closed-bioreactors: anti-cancer activity of the catechin-dextran conjugate, RSC Adv. 4(66) (2014) 35017-35026.

[323] O. Vittorio, G. Cirillo, F. Iemma, G. Di Turi, E. Jacchetti, M. Curcio, S. Barbuti, N. Funel, O.I. Parisi, F. Puoci, N. Picci, Dextran-Catechin Conjugate: A Potential Treatment Against the Pancreatic Ductal Adenocarcinoma, Pharmaceutical Res. 29(9) (2012) 2601-2614.

[324] O. Vittorio, V. Voliani, P. Faraci, B. Karmakar, F. Iemma, S. Hampel, M. Kavallaris, G. Cirillo, Magnetic catechin-dextran conjugate as targeted therapeutic for pancreatic tumour cells, J. Drug Targeting 22(5) (2014) 408-415.

[325] G. Cirillo, O. Vittorio, S. Hampel, F. Iemma, P. Parchi, M. Cecchini, F. Puoci, N. Picci, Quercetin nanocomposite as novel anticancer therapeutic: Improved efficiency and reduced toxicity, Eur. J. Pharmaceutical Sci. 49(3) (2013) 359-365.

[326] F. Puoci, C. Morelli, G. Cirillo, M. Curcio, O.I. Parisi, P. Maris, D. Sisci, N. Picci, Anticancer Activity of a Quercetin-based Polymer Towards HeLa Cancer Cells, Anticancer Res 32(7) (2012) 2843-2847.

[327] O. Vittorio, M. Brandl, G. Cirillo, U.G. Spizzirri, N. Picci, M. Kavallaris, F. Iemma, S. Hampel, Novel functional cisplatin carrier based on carbon nanotubes-quercetin nanohybrid induces synergistic anticancer activity against neuroblastoma in vitro, RSC Adv. 4(59) (2014) 31378-31384.

[328] G. Cirillo, O. Vittorio, S. Hampel, U.G. Spizzirri, N. Picci, F. Iemma, Incorporation of carbon nanotubes into a gelatin-catechin conjugate: Innovative approach for the preparation of anticancer materials, Int. J. Pharmaceutics 446(1-2) (2013) 176-182.

[329] M. Elsabahy, G.S. Heo, S.-M. Lim, G. Sun, K.L. Wooley, Polymeric Nanostructures for Imaging and Therapy, Chemical Reviews 115(19) (2015) 10967-11011.

[330] Y. Matsumura, H. Maeda, A New Concept for Macromolecular Therapeutics in Cancer Chemotherapy: Mechanism of Tumoritropic Accumulation of Proteins and the Antitumor Agent Smancs, Cancer Research 46(12 Part 1) (1986) 6387.

[331] H. Nakamura, F. Jun, H. Maeda, Development of next-generation macromolecular drugs based on the EPR effect: challenges and pitfalls, Expert Opinion on Drug Delivery 12(1) (2015) 53-64.

[332] H. Kobayashi, P.L. Choyke, Super enhanced permeability and retention (SUPR) effects in tumors following near infrared photoimmunotherapy, Nanoscale 8(25) (2016) 12504-12509.

[333] T. Nagaya, Y. Nakamura, K. Sato, T. Harada, P.L. Choyke, H. Kobayashi, Improved micro-distribution of antibody-photon absorber conjugates after initial near infrared photoimmunotherapy (NIR-PIT), Journal of Controlled Release 232 (2016) 1-8.

[334] Y. Nakamura, A. Mochida, P.L. Choyke, H. Kobayashi, Nanodrug Delivery: Is the Enhanced Permeability and Retention Effect Sufficient for Curing Cancer?, Bioconjugate Chemistry 27(10) (2016) 2225-2238.

[335] W.G. Kreyling, A.M. Abdelmonem, Z. Ali, F. Alves, M. Geiser, N. Haberl, R. Hartmann, S. Hirn, D.J. de Aberasturi, K. Kantner, G. Khadem-Saba, J.-M. Montenegro, J. Rejman, T. Rojo, I.R. de Larramendi, R. Ufartes, A. Wenk, W.J. Parak, In vivo integrity of polymer-coated gold nanoparticles, Nat. Nanotechnol. 10(7) (2015) 619-623. 
[336] M. Kawashita, R. Shineha, H.-M. Kim, T. Kokubo, Y. Inoue, N. Araki, Y. Nagata, M. Hiraoka, Y. Sawada, Preparation of ceramic microspheres for in situ radiotherapy of deep-seated cancer, Biomaterials 24(17) (2003) 2955-2963.

[337] N.H. Nicolay, D.P. Berry, R.A. Sharma, Liver metastases from colorectal cancer: radioembolization with systemic therapy, Nat Rev Clin Oncol 6(12) (2009) 687-697.

[338] M.A. Savin, M. Chehab, J.M. Campbell, J.H. Savin, C. Cash, C.-y.O. Wong, C.C. Schultz, Yttrium-90 Infusion: Incidence and Outcome of Delivery System Occlusions during 885 Deliveries, Journal of Vascular and Interventional Radiology 26(12) (2015) 1769-1776.

[339] M. Ochs, S. Carregal-Romero, J. Rejman, K. Braeckmans, S.C. De Smedt, W.J. Parak, Light-Addressable Capsules as Caged Compound Matrix for Controlled Triggering of Cytosolic Reactions, Angew. Chem. Int. Ed. 52(2) (2013) 695-699.

[340] B. Zebli, A.S. Susha, G.B. Sukhorukov, A.L. Rogach, W.J. Parak, Magnetic Targeting and Cellular Uptake of Polymer Microcapsules Simultaneously Functionalized with Magnetic and Luminescent Nanocrystals, Langmuir 21(10) (2005) 4262-4265.

[341] E. Lueshen, I. Venugopal, T. Soni, A. Alaraj, A. Linninger, Implant-assisted intrathecal magnetic drug targeting to aid in therapeutic nanoparticle localization for potential treatment of central nervous system disorders, J. Biomed. Nanotechnol. 11(2) (2015) 253-261.

[342] W. He, Y. Ji, C. Luo, Z. Xu, Development of single-side magnet array for super paramagnetic nano-particle targeting, Res. J. Appl. Sci., Eng. Technol. 7(15) (2014) 3022-3029, 8.

[343] A. Nacev, I.N. Weinberg, P.Y. Stepanov, S. Kupfer, L.O. Mair, M.G. Urdaneta, M. Shimoji, S.T. Fricke, B. Shapiro, Dynamic Inversion Enables External Magnets To Concentrate Ferromagnetic Rods to a Central Target, Nano Lett. 15(1) (2015) 359-364.

[344] S. Taherkhani, M. Mohammadi, J. Daoud, S. Martel, M. Tabrizian, Covalent Binding of Nanoliposomes to the Surface of Magnetotactic Bacteria for the Synthesis of Self-Propelled Therapeutic Agents, ACS nano 8(5) (2014) 5049-5060.

[345] R. Tietze, S. Lyer, S. Durr, T. Struffert, T. Engelhorn, M. Schwarz, E. Eckert, T. Goen, S. Vasylyev, W. Peukert, F. Wiekhorst, L. Trahms, A. Dorfler, C. Alexiou, Efficient drug-delivery using magnetic nanoparticles--biodistribution and therapeutic effects in tumour bearing rabbits, Nanomed. 9(7) (2013) 961-71.

[346] A. Gilbert, L. Ziegler, M. Martland, S. Davidson, F. Efficace, D. Sebag-Montefiore, G. Velikova, Systematic Review of Radiation Therapy Toxicity Reporting in Randomized Controlled Trials of Rectal Cancer: A Comparison of Patient-Reported Outcomes and Clinician Toxicity Reporting, International Journal of Radiation Oncology*Biology*Physics 92(3) (2015) $555-567$.

[347] M. Loos, P. Quentmeier, T. Schuster, U. Nitsche, R. Gertler, A. Keerl, T. Kocher, H. Friess, R. Rosenberg, Effect of Preoperative Radio(chemo)therapy on Long-term Functional Outcome in Rectal Cancer Patients: A Systematic Review and Meta-analysis, Annals of Surgical Oncology 20(6) (2013) 1816-1828.

[348] J. Ng, I. Shuryak, Minimizing second cancer risk following radiotherapy: current perspectives, Cancer Management and Research 7 (2015) 1-11.

[349] R. Rivoirard, A. Vallard, J. Langrand-Escure, M. Ben Mrad, G. Wang, J.-B. Guy, P. Diao, A. Dubanchet, E. Deutsch, C. Rancoule, N. Magne, Thirty years of phase I radiochemotherapy trials: Latest development, European journal of cancer 58 (2016) 1-7.

[350] J. Zhao, Y. Xia, J. Kaminski, Z. Hao, F. Mott, J. Campbell, R. Sadek, F.-M. Kong, Treatment-Related Death during Concurrent Chemoradiotherapy for Locally Advanced Non- 
Small Cell Lung Cancer: A Meta-Analysis of Randomized Studies, PloS one 11(6) (2016) e0157455.

[351] L.-L. Zhu, L. Yuan, H. Wang, L. Ye, G.-Y. Yao, C. Liu, N.-N. Sun, X.-J. Li, S.-C. Zhai, L.-J. Niu, J.-B. Zhang, H.-L. Ji, X.-M. Li, A Meta-Analysis of Concurrent Chemoradiotherapy for Advanced Esophageal Cancer, PloS one 10(6) (2015) e0128616.

[352] H. Bunjes, Lipid nanoparticles for the delivery of poorly water-soluble drugs, J. Pharm. Pharmacol. 62(11) (2010) 1637-1645.

[353] R. Li, S.-J. Lim, H.-G. Choi, M.-K. Lee, Solid lipid nanoparticles as drug delivery system for water-insoluble drugs, J. Pharm. Invest. 40 (2010) 63-73.

[354] Y.N. Usha, T.T. Angel, N. Udupa, Nanotechnology: perspectives on solubility/bioavailability enhancement, Pharma Rev. 8(45) (2010) 59-66.

[355] Q.A. Pankhurst, N.K.T. Thanh, S.K. Jones, J. Dobson, Progress in applications of magnetic nanoparticles in biomedicine, J. Phys. D-Appl. Phys. 42(22) (2009) 224001.

[356] M.F. Casula, P. Floris, C. Innocenti, A. Lascialfari, M. Marinone, M. Corti, R.A. Sperling, W.J. Parak, C. Sangregorio, Magnetic Resonance Imaging Contrast Agents Based on Iron Oxide Superparamagnetic Ferrofluids, Chem. Mater. 22(5) (2010) 1739-1748.

[357] I. Brigger, J. Morizet, L. Laudani, G. Aubert, M. Appel, V. Velasco, M.J. Terrier-Lacombe, D. Desmaele, J. d'Angelo, P. Couvreur, G. Vassal, Negative preclinical results with stealth((R)) nanospheres-encapsulated Doxorubicin in an orthotopic murine brain tumor model, J. Control. Rel. 100(1) (2004) 29-40.

[358] G. Storm, S.O. Belliot, T. Daemen, D.D. Lasic, Surface Modification of Nanoparticles to Oppose Uptake by the Mononuclear Phagocyte System, Adv. Drug Deliver. Rev. 17(1) (1995) 31-48.

[359] C. Alexiou, R. Jurgons, R.J. Schmid, C. Bergemann, J. Henke, W. Erhardt, E. Huenges, F. Parak, Magnetic drug targeting - Biodistribution of the magnetic carrier and the chemotherapeutic agent mitoxantrone after locoregional cancer treatment, J. Drug Target 11(3) (2003) 139-149.

[360] S. Lyer, E. Schreiber, R. Tietze, J. Mann, T. Struffert, T. Engelhorn, H. Rahn, K. Gitter, F. Wiekhorst, U. Steinhoff, L. Trahms, S. Odenbach, A. Dorfler, M. Ostermeier, S. Britzen, W. Schmidt, R. Hellinger, C. Alexiou, Nanotechnology and Cancer Treatment: Magnetic Nanoparticles for a New and Innovative Drug Delivery System, Anticancer Res 31(5) (2011) 1991-1992.

[361] R. Tietze, J. Zaloga, H. Unterweger, S. Lyer, R.P. Friedrich, C. Janko, M. Pottler, S. Durr, C. Alexiou, Magnetic nanoparticle-based drug delivery for cancer therapy, Biochem. Biophys. Res. Commun. 468(3) (2015) 463-70.

[362] T. Wang, M. Brewer, Q. Zhu, An overview of optical coherence tomography for ovarian tissue imaging and characterization, Wiley Interdisciplinary Reviews: Nanomedicine and Nanobiotechnology 7(1) (2015) 1-16.

[363] R.E. Wijesinghe, K. Park, D.-H. Kim, M. Jeon, J. Kim, In vivo imaging of melanomaimplanted magnetic nanoparticles using contrast-enhanced magneto-motive optical Doppler tomography, Journal of biomedical optics 21(6) (2016) 064001-064001.

[364] C. Alexiou, R.J. Schmid, R. Jurgons, M. Kremer, G. Wanner, C. Bergemann, E. Huenges, T. Nawroth, W. Arnold, F.G. Parak, Targeting cancer cells: magnetic nanoparticles as drug carriers, Eur. Biophys. J. Biophys. Lett. 35(5) (2006) 446-450.

[365] C. Alexiou, W. Arnold, P. Hulin, R.J. Klein, H. Renz, F.G. Parak, C. Bergemann, A.S. Lubbe, Magnetic mitoxantrone nanoparticle detection by histology, X-ray and MRI after magnetic tumor targeting, J. Magnetism and Magnetic Mater. 225(1-2) (2001) 187-193. 
[366] C. Alexiou, R. Jurgons, R. Schmid, W. Erhardt, F. Parak, C. Bergemann, H. Iro, Magnetic Drug Targeting - A new approach in locoregional tumortherapy with chemotherapeutic agents. Experimental animal studies, HNO 53(7) (2005) 618-622.

[367] H. Rahn, I. Gomez-Morilla, R. Jurgons, C. Alexiou, S. Odenbach, Microcomputed tomography analysis of ferrofluids used for cancer treatment, J. Phys. Cond. Matt. 20(20) (2008) 204152-204156.

[368] C.-C. Huang, Z.-X. Liao, H.-M. Lu, W.-Y. Pan, W.-L. Wan, C.-C. Chen, H.-W. Sung, Cellular Organelle-Dependent Cytotoxicity of Iron Oxide Nanoparticles and Its Implications for Cancer Diagnosis and Treatment: A Mechanistic Investigation, Chemistry of Materials (2016).

[369] Y.-S. Lee, J. Jeong, Y.J. Kim, M.-C. Lee, E. Kwon, W.-J. Myeong, D.S. Lee, J.-K. Chung, M.C. Lee, Long term evaluation of 59Fe labeled iron oxide nanoparticles in vivo, J. Nucl. Med. Meeting Abstr. 53(1) (2012) 1547-.

[370] J. Zaloga, C. Janko, R. Agarwal, J. Nowak, R. Muller, A.R. Boccaccini, G. Lee, S. Odenbach, S. Lyer, C. Alexiou, Different storage conditions influence biocompatibility and physicochemical properties of iron oxide nanoparticles, Int. J. Mol. Sci. 16(5) (2015) 9368-84.

[371] J. Zaloga, M. Stapf, J. Nowak, M. Pottler, R.P. Friedrich, R. Tietze, S. Lyer, G. Lee, S. Odenbach, I. Hilger, C. Alexiou, Tangential Flow Ultrafiltration Allows Purification and Concentration of Lauric Acid-/Albumin-Coated Particles for Improved Magnetic Treatment, Int. J. Mol. Sci. 16(8) (2015) 19291-307.

[372] S.C. Baetke, T. Lammers, F. Kiessling, Applications of nanoparticles for diagnosis and therapy of cancer, Br. J. Radiol. (2015) 20150207.

[373] B. Wang, X. He, Z. Zhang, Y. Zhao, W. Feng, Metabolism of nanomaterials in vivo: blood circulation and organ clearance, Acc. Chem. Res. 46(3) (2013) 761-9.

[374] M.P. Monopoli, C. Åberg, A. Salvati, K.A. Dawson, Biomolecular coronas provide the biological identity of nanosized materials, Nat. Nanotechnol. 7(12) (2012) 779-86.

[375] P. Rivera-Gil, D. Jimenez De Aberasturi, V. Wulf, B. Pelaz, P. Del Pino, Y. Zhao, J.M. De La Fuente, I. Ruiz De Larramendi, T. Rojo, X.-J. Liang, W.J. Parak, The Challenge To Relate the Physicochemical Properties of Colloidal Nanoparticles to Their Cytotoxicity, Acc. Chem. Res. 46(3) (2013) 743-749.

[376] K. Pombo Garcia, K. Zarschler, L. Barbaro, J.A. Barreto, W. O'Malley, L. Spiccia, H. Stephan, B. Graham, Zwitterionic-coated "stealth" nanoparticles for biomedical applications: recent advances in countering biomolecular corona formation and uptake by the mononuclear phagocyte system, Small 10(13) (2014) 2516-29.

[377] M.I. Setyawati, C.Y. Tay, D. Docter, R.H. Stauber, D.T. Leong, Understanding and exploiting nanoparticles' intimacy with the blood vessel and blood, Chem. Soc. Rev. 44(22) (2015) 8174-8199.

[378] J. Lipka, M. Semmler-Behnke, R.A. Sperling, A. Wenk, S. Takenaka, C. Schleh, T. Kissel, W.J. Parak, W.G. Kreyling, Biodistribution of PEG-modified gold nanoparticles following intratracheal instillation and intravenous injection, Biomater. 31(25) (2010) 6574-6581.

[379] B. Pelaz, P. del Pino, P. Maffre, R. Hartmann, M. Gallego, S. Rivera-Fernández, J.M. de la Fuente, G.U. Nienhaus, W.J. Parak, Surface Functionalization of Nanoparticles with Polyethylene Glycol: Effects on Protein Adsorption and Cellular Uptake, ACS nano 9(7) (2015) 6996-7008.

[380] N. Feliu, D. Docter, M. Heine, P. del Pino, S. Ashraf, J. Kolosnjaj-Tabi, P. Macchiarini, P. Nielsen, D. Alloyeau, F. Gazeau, R.H. Stauber, W.J. Parak, In vivo degeneration and the fate of inorganic nanoparticles, Chem. Soc. Rev. 45(9) (2016) 2440-2457. 
[381] M. Chanana, P. Rivera_Gil, M.A. Correa-Duarte, L.M. Liz-Marzán, W.J. Parak, Physicochemical Properties of Protein-Coated Gold Nanoparticles in Biological Fluids and Cells before and after Proteolytic Digestion, Angew. Chem. Int. Ed. 52(15) (2013) 4179-4183.

[382] S.J. Soenen, W.J. Parak, J. Rejman, B. Manshian, (Intra)Cellular Stability of Inorganic Nanoparticles: Effects on Cytotoxicity, Particle Functionality, and Biomedical Applications, Chem. Rev. 115(5) (2015) 2109-2135.

[383] K. Zarschler, L. Rocks, N. Licciardello, L. Boselli, E. Polo, K.P. Garcia, L. De Cola, H. Stephan, K.A. Dawson, Ultrasmall inorganic nanoparticles: State-of-the-art and perspectives for biomedical applications, Nanomed. (2016).

[384] B.H. Kim, M.J. Hackett, J. Park, T. Hyeon, Synthesis, characterization, and application of ultrasmall nanoparticles, Chem. Mater. 26(1) (2014) 59-71.

[385] H. Soo Choi, W. Liu, P. Misra, E. Tanaka, J.P. Zimmer, B. Itty Ipe, M.G. Bawendi, J.V. Frangioni, Renal clearance of quantum dots, Nat. Biotechnol. 25(10) (2007) 1165-1170.

[386] W. Möller, N. Gibson, M. Geiser, S. Pokhrel, A. Wenk, S. Takenaka, O. Schmid, A. Bulgheroni, F. Simonelli, J. Kozempel, U. Holzwarth, C. Wigge, S. Eigeldinger-Berthou, L. Mädler, W.G. Kreyling, Gold nanoparticle aerosols for rodent inhalation and translocation studies, Journal of Nanoparticle Research 15(4) (2013) 1-13.

[387] S. Takenaka, W. Möller, M. Semmler-Behnke, E. Karg, A. Wenk, O. Schmid, T. Stoeger, L. Jennen, M. Aichler, A. Walch, S. Pokhrel, L. Mädler, O. Eickelberg, W.G. Kreyling, Efficient internalization and intracellular translocation of inhaled gold nanoparticles in rat alveolar macrophages, Nanomedicine 7(6) (2012) 855-865.

[388] X.D. Zhang, J. Chen, Z. Luo, D. Wu, X. Shen, S.S. Song, Y.M. Sun, P.X. Liu, J. Zhao, S. Huo, S. Fan, F. Fan, X.J. Liang, J. Xie, Enhanced tumor accumulation of sub-2 nm gold nanoclusters for cancer radiation therapy, Adv. Healthc. Mater. 3(1) (2014) 133-41.

[389] X.D. Zhang, Z. Luo, J. Chen, S. Song, X. Yuan, X. Shen, H. Wang, Y. Sun, K. Gao, L. Zhang, S. Fan, D.T. Leong, M. Guo, J. Xie, Ultrasmall glutathione-protected gold nanoclusters as next generation radiotherapy sensitizers with high tumor uptake and high renal clearance, Sci. Rep. 5 (2015) 8669.

[390] G. Le Duc, I. Miladi, C. Alric, P. Mowat, E. Brauer-Krisch, A. Bouchet, E. Khalil, C. Billotey, M. Janier, F. Lux, T. Epicier, P. Perriat, S. Roux, O. Tillement, Toward an imageguided microbeam radiation therapy using gadolinium-based nanoparticles, ACS nano 5(12) (2011) 9566-74.

[391] P. Mowat, A. Mignot, W. Rima, F. Lux, O. Tillement, C. Roulin, M. Dutreix, D. Bechet, S. Huger, L. Humbert, M. Barberi-Heyob, M.T. Aloy, E. Armandy, C. Rodriguez-Lafrasse, G. Le Duc, S. Roux, P. Perriat, In vitro radiosensitizing effects of ultrasmall gadolinium based particles on tumour cells, J. Nanosci. Nanotechnol. 11(9) (2011) 7833-9.

[392] I. Miladi, G.L. Duc, D. Kryza, A. Berniard, P. Mowat, S. Roux, J. Taleb, P. Bonazza, P. Perriat, F. Lux, O. Tillement, C. Billotey, M. Janier, Biodistribution of ultra small gadoliniumbased nanoparticles as theranostic agent: application to brain tumors, J. Biomater. Appl. 28(3) (2013) 385-94.

[393] G. Le Duc, S. Roux, A. Paruta-Tuarez, S. Dufort, E. Brauer, A. Marais, C. Truillet, L. Sancey, P. Perriat, F. Lux, O. Tillement, Advantages of gadolinium based ultrasmall nanoparticles vs molecular gadolinium chelates for radiotherapy guided by MRI for glioma treatment, Cancer Nanotechnol. 5(1) (2014) 4.

[394] L. Sancey, F. Lux, S. Kotb, S. Roux, S. Dufort, A. Bianchi, Y. Cremillieux, P. Fries, J.L. Coll, C. Rodriguez-Lafrasse, M. Janier, M. Dutreix, M. Barberi-Heyob, F. Boschetti, F. Denat, C. Louis, E. Porcel, S. Lacombe, G. Le Duc, E. Deutsch, J.L. Perfettini, A. Detappe, C. Verry, R. 
Berbeco, K.T. Butterworth, S.J. McMahon, K.M. Prise, P. Perriat, O. Tillement, The use of theranostic gadolinium-based nanoprobes to improve radiotherapy efficacy, Br. J. Radiol. 87(1041) (2014) 20140134.

[395] I. Miladi, M.T. Aloy, E. Armandy, P. Mowat, D. Kryza, N. Magne, O. Tillement, F. Lux, C. Billotey, M. Janier, C. Rodriguez-Lafrasse, Combining ultrasmall gadolinium-based nanoparticles with photon irradiation overcomes radioresistance of head and neck squamous cell carcinoma, Nanomed. 11(1) (2015) 247-57.

[396] L. Sancey, S. Kotb, C. Truillet, F. Appaix, A. Marais, E. Thomas, B. van der Sanden, J.P. Klein, B. Laurent, M. Cottier, R. Antoine, P. Dugourd, G. Panczer, F. Lux, P. Perriat, V. MottoRos, O. Tillement, Long-term in vivo clearance of gadolinium-based AGuIX nanoparticles and their biocompatibility after systemic injection, ACS nano 9(3) (2015) 2477-88.

[397] F. Taupin, M. Flaender, R. Delorme, T. Brochard, J.F. Mayol, J. Arnaud, P. Perriat, L. Sancey, F. Lux, R.F. Barth, M. Carriere, J.L. Ravanat, H. Elleaume, Gadolinium nanoparticles and contrast agent as radiation sensitizers, Phys. Med. Biol. 60(11) (2015) 4449-64.

[398] F. Lux, A. Mignot, P. Mowat, C. Louis, S. Dufort, C. Bernhard, F. Denat, F. Boschetti, C. Brunet, R. Antoine, P. Dugourd, S. Laurent, L. Vander Elst, R. Muller, L. Sancey, V. Josserand, J.L. Coll, V. Stupar, E. Barbier, C. Remy, A. Broisat, C. Ghezzi, G. Le Duc, S. Roux, P. Perriat, O. Tillement, Ultrasmall rigid particles as multimodal probes for medical applications, Angew. Chem. Int. Ed. Engl. 50(51) (2011) 12299-303.

[399] R. Bazak, M. Houri, S. El Achy, S. Kamel, T. Refaat, Cancer active targeting by nanoparticles: a comprehensive review of literature, J. Cancer Res. Clin. Oncol. 141(5) (2015) 769-84.

[400] S. Joshi, I. Ghosh, S. Pokhrel, L. Mädler, W.M. Nau, Interactions of Amino Acids and Polypeptides with Metal Oxide Nanoparticles Probed by Fluorescent Indicator Adsorption and Displacement, ACS nano 6(6) (2012) 5668-5679.

[401] J. Choi, Y. Park, E.B. Choi, H.O. Kim, D.J. Kim, Y. Hong, S.H. Ryu, J.H. Lee, J.S. Suh, J. Yang, Y.M. Huh, S. Haam, Aptamer-conjugated gold nanorod for photothermal ablation of epidermal growth factor receptor-overexpressed epithelial cancer, J. Biomed. Opt. 19(5) (2014) 051203.

[402] J.V. Jokerst, Z. Miao, C. Zavaleta, Z. Cheng, S.S. Gambhir, Affibody-functionalized goldsilica nanoparticles for Raman molecular imaging of the epidermal growth factor receptor, Small 7(5) (2011) 625-33.

[403] M.P. Melancon, M. Zhou, R. Zhang, C. Xiong, P. Allen, X. Wen, Q. Huang, M. Wallace, J.N. Myers, R.J. Stafford, D. Liang, A.D. Ellington, C. Li, Selective uptake and imaging of aptamer- and antibody-conjugated hollow nanospheres targeted to epidermal growth factor receptors overexpressed in head and neck cancer, ACS nano 8(5) (2014) 4530-8.

[404] B.G. Ongarora, K.R. Fontenot, X. Hu, I. Sehgal, S.D. Satyanarayana-Jois, M.G. Vicente, Phthalocyanine-peptide conjugates for epidermal growth factor receptor targeting, J. Med. Chem. 55(8) (2012) 3725-38.

[405] K. Zarschler, K. Prapainop, E. Mahon, L. Rocks, M. Bramini, P.M. Kelly, H. Stephan, K.A. Dawson, Diagnostic nanoparticle targeting of the EGF-receptor in complex biological conditions using single-domain antibodies, Nanoscale 6(11) (2014) 6046-56.

[406] K. Zarschler, K. Zscheppang, F. Kapplusch, N. Cordes, H. Stephan, Single-domain antibodies: Next-generation targeting vectors for molecular imaging, Nucl. Med. Biol. 41(7) (2014) 628-628. 
[407] J.-M. Montenegro, V. Grazu, A. Sukhanova, S. Agarwal, J.M. de la Fuente, I. Nabiev, A. Greiner, W.J. Parak, Controlled antibody/(bio-) conjugation of inorganic nanoparticles for targeted delivery, Adv. Drug Deliv. Rev. 65(5) (2013) 677-688.

[408] A. Nel, T. Xia, L. Mädler, N. Li, Toxic Potential of Materials at the Nanolevel, Science 311(5761) (2006) 622-627.

[409] A.E. Nel, L. Madler, D. Velegol, T. Xia, E.M.V. Hoek, P. Somasundaran, F. Klaessig, V. Castranova, M. Thompson, Understanding biophysicochemical interactions at the nano-bio interface, Nat. Mater. 8(7) (2009) 543-557.

[410] W.G. Kreyling, S. Hirn, W. Möller, C. Schleh, A. Wenk, G. Celik, J. Lipka, M. Schäffler, N. Haberl, B.D. Johnston, R. Sperling, G. Schmid, U. Simon, W.J. Parak, M. Semmler-Behnke, Air-Blood Barrier Translocation of Tracheally Instilled Gold Nanoparticles Inversely Depends on Particle Size, ACS nano 8(1) (2014) 222-233.

[411] K.T. Butterworth, J.A. Coulter, S. Jain, J. Forker, S.J. McMahon, G. Schettino, K.M. Prise, F.J. Currell, D.G. Hirst, Evaluation of cytotoxicity and radiation enhancement using $1.9 \mathrm{~nm}$ gold particles: potential application for cancer therapy, Nanotechnol. 21(29) (2010) 295101.

[412] S. Jain, J.A. Coulter, A.R. Hounsell, K.T. Butterworth, S.J. McMahon, W.B. Hyland, M.F. Muir, G.R. Dickson, K.M. Prise, F.J. Currell, J.M. O'Sullivan, D.G. Hirst, Cell-specific radiosensitization by gold nanoparticles at megavoltage radiation energies, Int. J. Rad. Oncol. Biol. Phys. 79(2) (2011) 531-9.

[413] L.E. Taggart, S.J. McMahon, F.J. Currell, K.M. Prise, K.T. Butterworth, The role of mitochondrial function in gold nanoparticle mediated radiosensitisation, Cancer Nanotechnol. 5(1) (2014) 5.

[414] H. Zhang, S. Pokhrel, Z. Ji, H. Meng, X. Wang, S. Lin, C.H. Chang, L. Li, R. Li, B. Sun, M. Wang, Y.-P. Liao, R. Liu, T. Xia, L. Mädler, A.E. Nel, PdO Doping Tunes Band-Gap Energy Levels as Well as Oxidative Stress Responses to a Co3O4 p-Type Semiconductor in Cells and the Lung, J. Am. Chem. Soc. 136(17) (2014) 6406-6420.

[415] J.A.H. Dreyer, S. Pokhrel, J. Birkenstock, M.G. Hevia, M. Schowalter, A. Rosenauer, A. Urakawa, W.Y. Teoh, L. Madler, Decrease of the required dopant concentration for d-Bi2O3 crystal stabilization through thermal quenching during single-step flame spray pyrolysis, CrystEngComm 18(12) (2016) 2046-2056.

[416] H.K. Kammler, L. Mädler, S.E. Pratsinis, Flame Synthesis of Nanoparticles, Chem. Eng. Technol. 24(6) (2001) 583-596.

[417] J.A. Kemmler, S. Pokhrel, J. Birkenstock, M. Schowalter, A. Rosenauer, N. Bârsan, U. Weimar, L. Mädler, Quenched, nanocrystalline In4Sn3O12 high temperature phase for gas sensing applications, Sens. Actuators B: Chem. 161(1) (2012) 740-747.

[418] J.A. Kemmler, S. Pokhrel, L. Mädler, U. Weimar, N. Barsan, Flame spray pyrolysis for sensing at the nanoscale, Nanotechnol. 24(44) (2013) 442001.

[419] M. Minnermann, H.K. Grossmann, S. Pokhrel, K. Thiel, H. Hagelin-Weaver, M. Bäumer, L. Mädler, Double flame spray pyrolysis as a novel technique to synthesize alumina-supported cobalt Fischer-Tropsch catalysts, Cat.Today 214(0) (2013) 90-99.

[420] M. Minnermann, S. Pokhrel, K. Thiel, R. Henkel, J. Birkenstock, T. Laurus, A. Zargham, J.-I. Flege, V. Zielasek, E. Piskorska-Hommel, J. Falta, L. Mädler, M. Bäumer, Role of Palladium in Iron Based Fischer-Tropsch Catalysts Prepared by Flame Spray Pyrolysis†, J. Phys. Chem. C 115(4) (2010) 1302-1310.

[421] S. Pokhrel, J. Birkenstock, M. Schowalter, A. Rosenauer, L. MaÌ dler, Growth of Ultrafine Single Crystalline WO3 Nanoparticles Using Flame Spray Pyrolysis, Crystal Growth \& Design 10(2) (2010) 632-639. 
[422] W.Y. Teoh, R. Amal, L. Madler, Flame spray pyrolysis: An enabling technology for nanoparticles design and fabrication, Nanoscale 2(8) (2010) 1324-1347.

[423] A.M. Abdelmonem, B. Pelaz, K. Kantner, N.C. Bigall, P. del Pino, W.J. Parak, Charge and agglomeration dependent in vitro uptake and cytotoxicity of zinc oxide nanoparticles, J. Inorg. Biochem. 153 (2015) 334-338.

[424] S. Pokhrel, A.E. Nel, L. Madler, Custom-designed nanomaterial libraries for testing metal oxide toxicity, Acc. Chem. Res. 46(3) (2013) 632-41.

[425] A.D. Maynard, R.J. Aitken, T. Butz, V. Colvin, K. Donaldson, G. Oberdörster, M.A. Philbert, J. Ryan, A. Seaton, V. Stone, S.S. Tinkle, L. Tran, N.J. Walker, D.B. Warheit, Safe handling of nanotechnology, Nature 444(7117) (2006) 267-269.

[426] C. Torres-Duarte, A.S. Adeleye, S. Pokhrel, L. Mädler, A.A. Keller, G.N. Cherr, Developmental effects of two different copper oxide nanomaterials in sea urchin (Lytechinus pictus) embryos, Nanotoxicology 10(6) (2016) 671-679.

[427] J. Xiao, A. Kuc, S. Pokhrel, L. Mädler, R. Pöttgen, F. Winter, T. Frauenheim, T. Heine, FeDoped $\mathrm{ZnO}$ Nanoparticles: The Oxidation Number and Local Charge on Iron, Studied by 57Fe Mößbauer Spectroscopy and DFT Calculations, Chem. A Eur. J. 19(10) (2013) 3287-3291.

[428] J. Xiao, A. Kuc, S. Pokhrel, M. Schowalter, S. Parlapalli, A. Rosenauer, T. Frauenheim, L. Mädler, L.G.M. Pettersson, T. Heine, Evidence for Fe2+ in Wurtzite Coordination: Iron Doping Stabilizes ZnO Nanoparticles, Small 7(20) (2011) 2879-2886.

[429] R. Damoiseaux, S. George, M. Li, S. Pokhrel, Z. Ji, B. France, T. Xia, E. Suarez, R. Rallo, L. Madler, Y. Cohen, E.M.V. Hoek, A. Nel, No time to lose-high throughput screening to assess nanomaterial safety, Nanoscale 3(4) (2011) 1345-1360.

[430] S. George, S. Pokhrel, T. Xia, B. Gilbert, Z.X. Ji, M. Schowalter, A. Rosenauer, R. Damoiseaux, K.A. Bradley, L. Madler, A.E. Nel, Use of a Rapid Cytotoxicity Screening Approach To Engineer a Safer Zinc Oxide Nanoparticle through Iron Doping, ACS nano 4(1) (2010) 15-29.

[431] R. Li, Z. Ji, C.H. Chang, D.R. Dunphy, X. Cai, H. Meng, H. Zhang, B. Sun, X. Wang, J. Dong, S. Lin, M. Wang, Y.-P. Liao, C.J. Brinker, A. Nel, T. Xia, Surface Interactions with Compartmentalized Cellular Phosphates Explain Rare Earth Oxide Nanoparticle Hazard and Provide Opportunities for Safer Design, ACS nano 8(2) (2014) 1771-1783.

[432] V. Aruoja, S. Pokhrel, M. Sihtmae, M. Mortimer, L. Madler, A. Kahru, Toxicity of 12 metal-based nanoparticles to algae, bacteria and protozoa, Environ. Sci.: Nano 2(6) (2015) 630644.

[433] B. Sun, S. Pokhrel, D.R. Dunphy, H. Zhang, Z. Ji, X. Wang, M. Wang, Y.-P. Liao, C.H. Chang, J. Dong, R. Li, L. Mädler, C.J. Brinker, A.E. Nel, T. Xia, Reduction of Acute Inflammatory Effects of Fumed Silica Nanoparticles in the Lung by Adjusting Silanol Display through Calcination and Metal Doping, ACS nano 9(9) (2015) 9357-9372.

[434] B. Sun, X. Wang, Y.-P. Liao, Z. Ji, C.H. Chang, S. Pokhrel, J. Ku, X. Liu, M. Wang, D.R. Dunphy, R. Li, H. Meng, L. Mädler, C.J. Brinker, A.E. Nel, T. Xia, Repetitive Dosing of Fumed Silica Leads to Profibrogenic Effects through Unique Structure-Activity Relationships and Biopersistence in the Lung, ACS nano 10(8) (2016) 8054-8066.

[435] S.J. Soenen, P. Rivera-Gil, J.-M. Montenegro, W.J. Parak, S.C. De Smedt, K. Braeckmans, Cellular toxicity of inorganic nanoparticles: Common aspects and guidelines for improved nanotoxicity evaluation, Nano Today 6(5) (2011) 446-465.

[436] D.M. Blake, P.-C. Maness, Z. Huang, E.J. Wolfrum, J. Huang, Application of the photocatalytic chemistry of titanium dioxide to disinfetion and the killing of cancer cells, Separ. Purif. Method 28(1) (1999) 1-50. 
[437] T.L. Doane, C. Burda, The unique role of nanoparticles in nanomedicine: imaging, drug delivery and therapy, Chem. Soc. Rev. 41(7) (2012) 2885-2911.

[438] M. Valko, D. Leibfritz, J. Moncol, M.T.D. Cronin, M. Mazur, J. Telser, Free radicals and antioxidants in normal physiological functions and human disease, Int. J. Biochem. Cell Biol. 39(1) (2007) 44-84.

[439] A.E. Nel, W.J. Parak, W.C.W. Chan, T. Xia, M.C. Hersam, C.J. Brinker, J.I. Zink, K.E. Pinkerton, D.R. Baer, P.S. Weiss, Where Are We Heading in Nanotechnology Environmental Health and Safety and Materials Characterization?, ACS nano 9(6) (2015) 5627-5630.

[440] C.R. Thomas, S. George, A.M. Horst, Z. Ji, R.J. Miller, J.R. Peralta-Videa, T. Xia, S. Pokhrel, L. Mädler, J.L. Gardea-Torresdey, P.A. Holden, A.A. Keller, H.S. Lenihan, A.E. Nel, J.I. Zink, Nanomaterials in the Environment: From Materials to High-Throughput Screening to Organisms, ACS nano 5(1) (2011) 13-20.

[441] B. Gilbert, S.C. Fakra, T. Xia, S. Pokhrel, L. Mädler, A.E. Nel, The Fate of ZnO Nanoparticles Administered to Human Bronchial Epithelial Cells, ACS nano 6(6) (2012) 49214930.

[442] C. Kaweeteerawat, A. Ivask, R. Liu, H. Zhang, C.H. Chang, C. Low-Kam, H. Fischer, Z. Ji, S. Pokhrel, Y. Cohen, D. Telesca, J. Zink, L. Mädler, P.A. Holden, A. Nel, H. Godwin, Toxicity of Metal Oxide Nanoparticles in Escherichia coli Correlates with Conduction Band and Hydration Energies, Environmental Science \& Technology 49(2) (2015) 1105-1112.

[443] S. Lin, Y. Zhao, T. Xia, H. Meng, Z. Ji, R. Liu, S. George, S. Xiong, X. Wang, H. Zhang, S. Pokhrel, L. Mädler, R. Damoiseaux, S. Lin, A.E. Nel, High Content Screening in Zebrafish Speeds up Hazard Ranking of Transition Metal Oxide Nanoparticles, ACS nano 5(9) (2011) 7284-7295.

[444] S. Pokhrel, A.E. Nel, L. Mädler, Custom-Designed Nanomaterial Libraries for Testing Metal Oxide Toxicity, Acc. Chem. Res. 46(3) (2013) 632-641.

[445] H. Zhang, Z. Ji, T. Xia, H. Meng, C. Low-Kam, R. Liu, S. Pokhrel, S. Lin, X. Wang, Y.-P. Liao, M. Wang, L. Li, R. Rallo, R. Damoiseaux, D. Telesca, L. Mädler, Y. Cohen, J.I. Zink, A.E. Nel, Use of Metal Oxide Nanoparticle Band Gap To Develop a Predictive Paradigm for Oxidative Stress and Acute Pulmonary Inflammation, ACS nano 6(5) (2012) 4349-4368.

[446] S. George, S. Pokhrel, Z. Ji, B.L. Henderson, T. Xia, L. Li, J.I. Zink, A.E. Nel, L. Mädler, Role of Fe Doping in Tuning the Band Gap of $\mathrm{TiO} 2$ for the Photo-Oxidation-Induced Cytotoxicity Paradigm, J. Am. Chem. Soc. 133(29) (2011) 11270-11278.

[447] A. Mukherjee, S. Pokhrel, S. Bandyopadhyay, L. Mädler, J.R. Peralta-Videa, J.L. GardeaTorresdey, A soil mediated phyto-toxicological study of iron doped zinc oxide nanoparticles (Fe@ ZnO) in green peas (Pisum sativum L.), Chemical Engineering Journal 258 (2014) 394-401. [448] M. Schubert, S. Pokhrel, A. Thome, V. Zielasek, T.M. Gesing, F. Roessner, L. Madler, M. Baumer, Highly active Co-Al2O3-based catalysts for $\mathrm{CO} 2$ methanation with very low platinum promotion prepared by double flame spray pyrolysis, Catalysis Science \& Technology 6(20) (2016) 7449-7460.

[449] S. Pokhrel, J. Birkenstock, A. Dianat, J. Zimmermann, M. Schowalter, A. Rosenauer, L.C. Ciacchi, L. Madler, In situ high temperature X-ray diffraction, transmission electron microscopy and theoretical modeling for the formation of WO3 crystallites, CrystEngComm 17(36) (2015) 6985-6998.

[450] K.D. Kim, S. Pokhrel, Z. Wang, H. Ling, C. Zhou, Z. Liu, M. Hunger, L. Mädler, J. Huang, Tailoring High-Performance Pd Catalysts for Chemoselective Hydrogenation Reactions via Optimizing the Parameters of the Double-Flame Spray Pyrolysis, ACS Catalysis 6(4) (2016) 2372-2381. 
[451] Z. Wang, S. Pokhrel, M. Chen, M. Hunger, L. Mädler, J. Huang, Palladium-doped silicaalumina catalysts obtained from double-flame FSP for chemoselective hydrogenation of the model aromatic ketone acetophenone, Journal of Catalysis 302 (2013) 10-19.

[452] S.K. Singh, A.K. Singh, D. Kumar, O. Prakash, S.B. Rai, Efficient UV-visible upconversion emission in Er3+/Yb3+ co-doped La2O3 nano-crystalline phosphor, Appl. Phys. B 98(1) (2010) 173-179.

[453] I. Ali, U. Rahis, K. Salim, M.A. Rather, W.A. Wani, A. Haque, Adv. Nano Drugs Cancer Chemother., Curr. Cancer Drug Targets 11(2) (2011) 135-146.

[454] J. He, Y. Hu, M. Hu, B. Li, Development of PD-1/PD-L1 Pathway in Tumor Immune Microenvironment and Treatment for Non-Small Cell Lung Cancer, Sci. Rep. 5 (2015) 13110.

[455] C.N. Coleman, G.S. Higgins, J.M. Brown, M. Baumann, D.G. Kirsch, H. Willers, P.G.S. Prasanna, M.W. Dewhirst, E.J. Bernhard, M.M. Ahmed, Improving the Predictive Value of Preclinical Studies in Support of Radiotherapy Clinical Trials, Clinical Cancer Research 22(13) (2016) 3138.

[456] B.A. Ruggeri, F. Camp, S. Miknyoczki, Animal models of disease: pre-clinical animal models of cancer and their applications and utility in drug discovery, Biochem. Pharmacol. 87(1) (2014) 150-61.

[457] J. Jung, Human Tumor Xenograft Models for Preclinical Assessment of Anticancer Drug Development, Toxicol. Res. 30(1) (2014) 1-5.

[458] F.L. Byrne, J.A. McCarroll, M. Kavallaris, Analyses of Tumor Burden In Vivo and Metastasis Ex Vivo Using Luciferase-Expressing Cancer Cells in an Orthotopic Mouse Model of Neuroblastoma, Methods Mol. Biol. 1372 (2016) 61-77.

[459] L.D. Shultz, M.A. Brehm, J.V. Garcia-Martinez, D.L. Greiner, Humanized mice for immune system investigation: progress, promise and challenges, Nat. Rev. Immunol. 12(11) (2012) 786-798.

[460] O.P. Singh, S. Sundar, Immunotherapy and Targeted Therapies in Treatment of Visceral Leishmaniasis: Current Status and Future Prospects, Front. Immunol. 5 (2014) 296.

[461] C. Walesky, G. Edwards, P. Borude, S. Gunewardena, M. O'Neil, B. Yoo, U. Apte, Hepatocyte nuclear factor 4 alpha deletion promotes diethylnitrosamine-induced hepatocellular carcinoma in rodents, Hepatol. 57(6) (2013) 2480-90.

[462] N.S. Yaacob, H.M. Yankuzo, S. Devaraj, J.K. Wong, C.S. Lai, Anti-Tumor Action, Clinical Biochemistry Profile and Phytochemical Constituents of a Pharmacologically Active Fraction of S. crispus in NMU-Induced Rat Mammary Tumour Model, PloS one 10(5) (2015) $\mathrm{e} 0126426$

[463] A.A. Zeeneldin, H. Ramadan, M.M. El Gammal, M.M. Saber, D. Elgamal, M.A. Sherisher, Gastric carcinoma at Tanta Cancer Center: A comparative retrospective clinico-pathological study of the elderly versus the non-elderly, J. Egyp. Natl. Cancer Inst. 26(3) (2014) 127-137.

[464] K.R. Greenow, M.J. Smalley, Overview of Genetically Engineered Mouse Models of Breast Cancer Used in Translational Biology and Drug Development, Curr. Protoc. Pharmacol. 70 (2015) 1436 1-14.

[465] C.-P. Day, G. Merlino, T. Van Dyke, Preclinical Mouse Cancer Models: A Maze of Opportunities and Challenges, Cell 163(1) (2015) 39-53.

[466] K. Jin, L. Teng, Y. Shen, K. He, Z. Xu, G. Li, Patient-derived human tumour tissue xenografts in immunodeficient mice: a systematic review, Clin. Transl. Oncol. 12(7) (2010) 47380 .

[467] D. Siolas, G.J. Hannon, Patient-derived tumor xenografts: transforming clinical samples into mouse models, Cancer Res. 73(17) (2013) 5315-9. 
[468] A. Wicki, D. Witzigmann, V. Balasubramanian, J. Huwyler, Nanomedicine in cancer therapy: Challenges, opportunities, and clinical applications, J. Control. Rel. 200 (2015) 138-157. [469] S. Dhar, Z. Liu, J. Thomale, H. Dai, S.J. Lippard, Targeted Single-Wall Carbon NanotubeMediated Pt(IV) Prodrug Delivery Using Folate as a Homing Device, J. Am. Chem. Soc. 130(34) (2008) 11467-11476.

[470] T.R. Fadel, E.R. Steenblock, E. Stern, N. Li, X. Wang, G.L. Haller, L.D. Pfefferle, T.M. Fahmy, Enhanced Cellular Activation with Single Walled Carbon Nanotube Bundles Presenting Antibody Stimuli, Nano Lett. 8(7) (2008) 2070-2076.

[471] P.M. Ajayan, J.M. Tour, Mater. Sci.: Nanotube composites, Nature 447(7148) (2007) 1066-1068. 


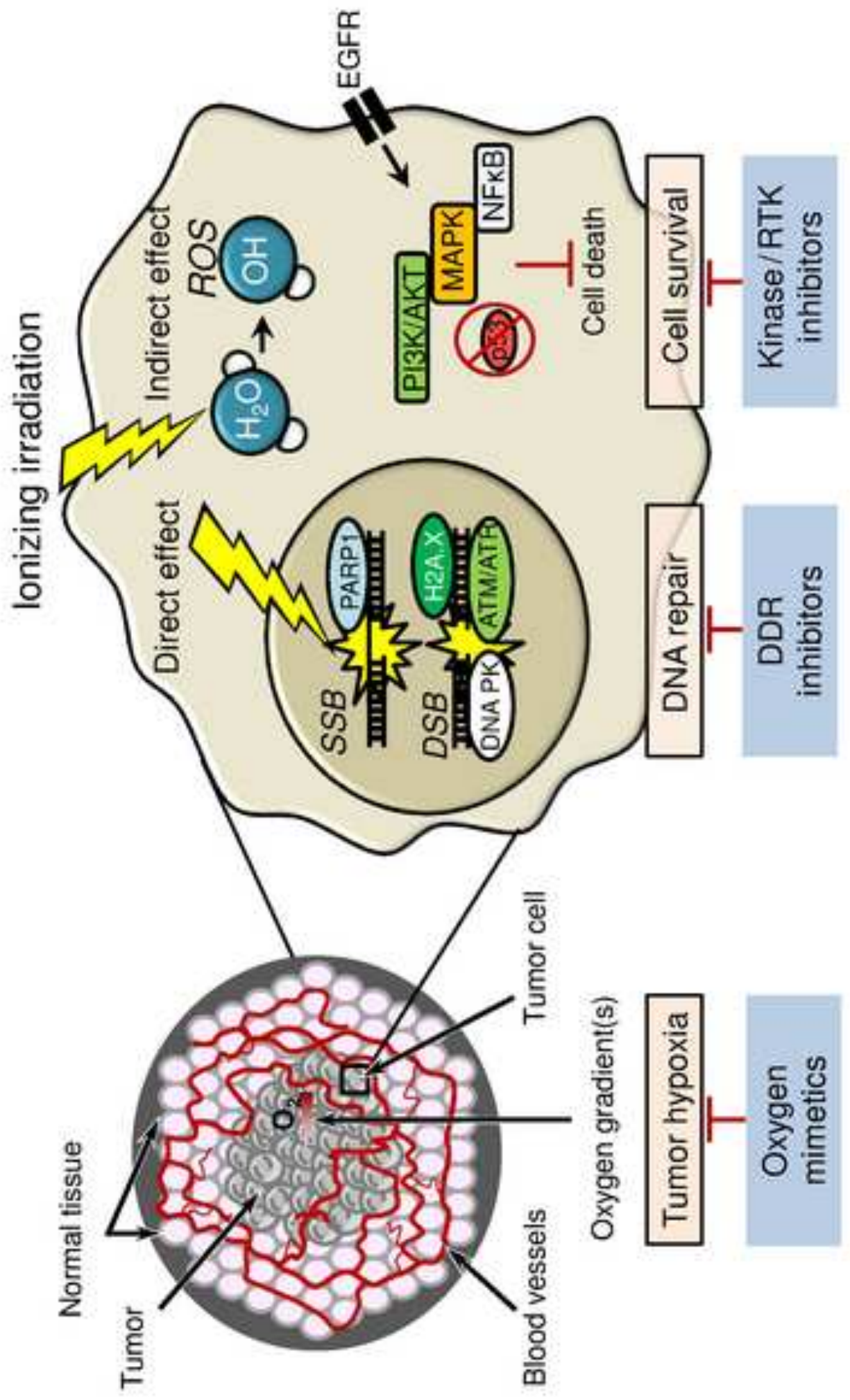




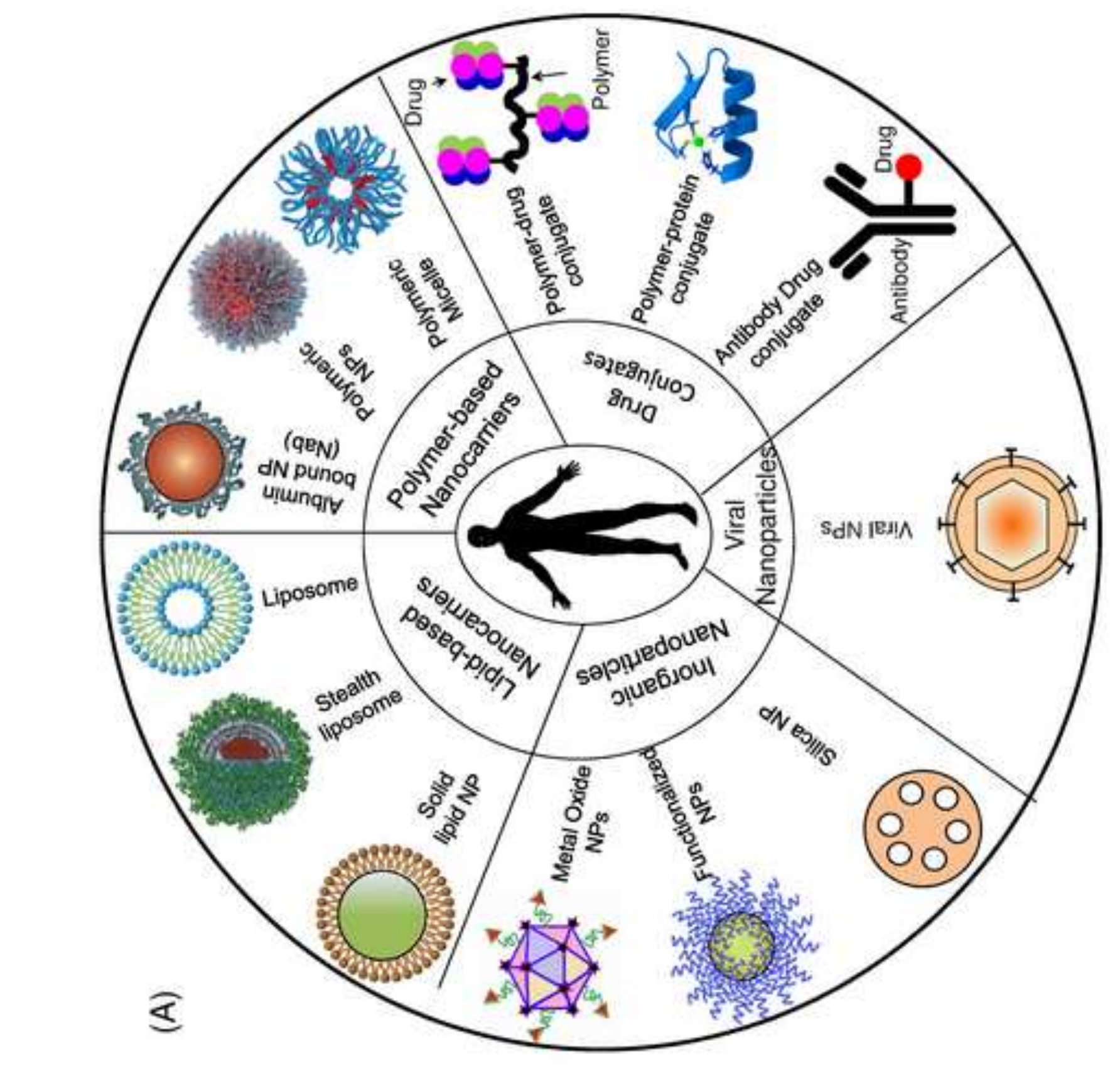

Figure 2(A)

of 


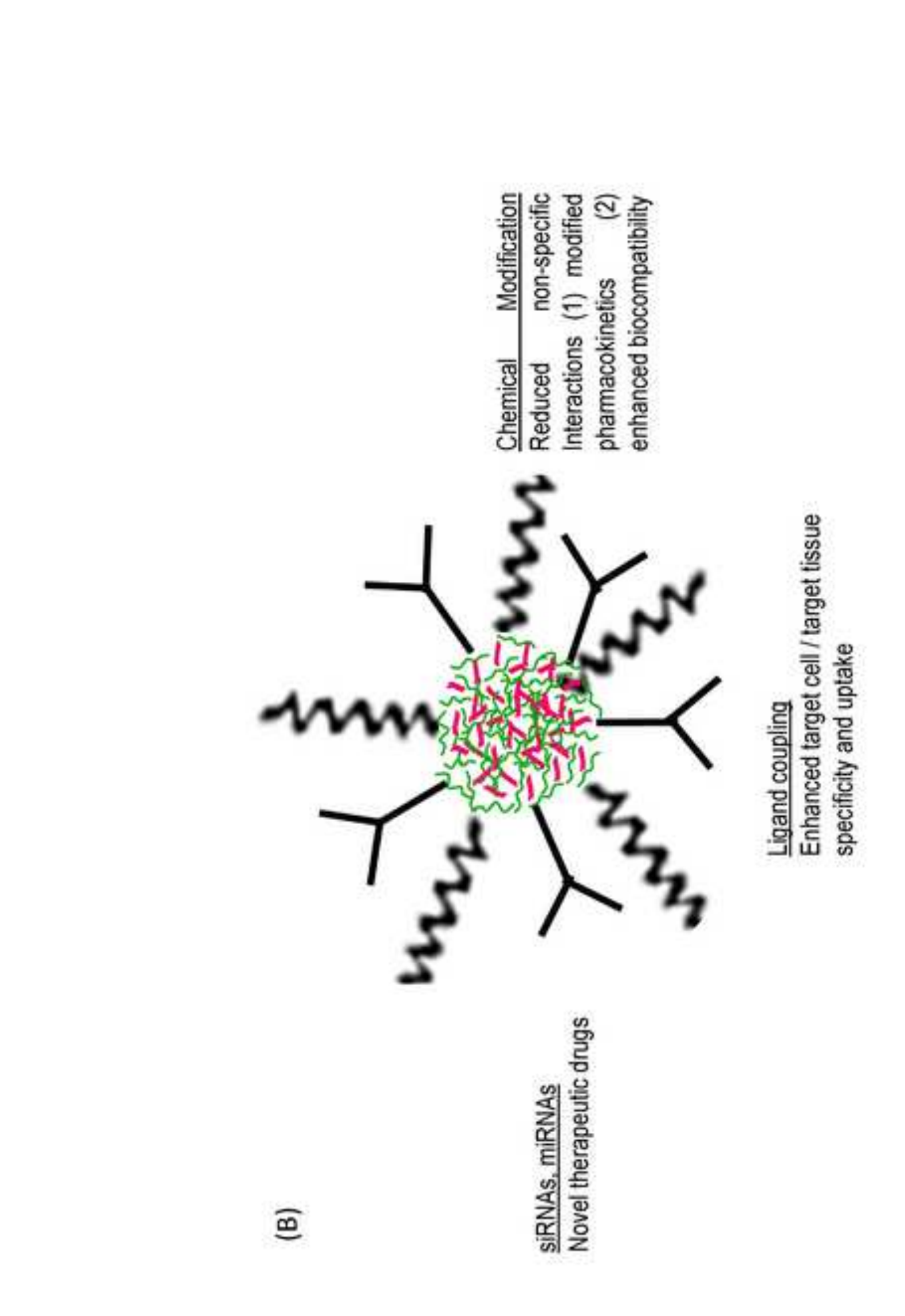

$\bar{\emptyset}$

Figure 2(B)

$$
\text { . }
$$$$
\text { (10) }
$$
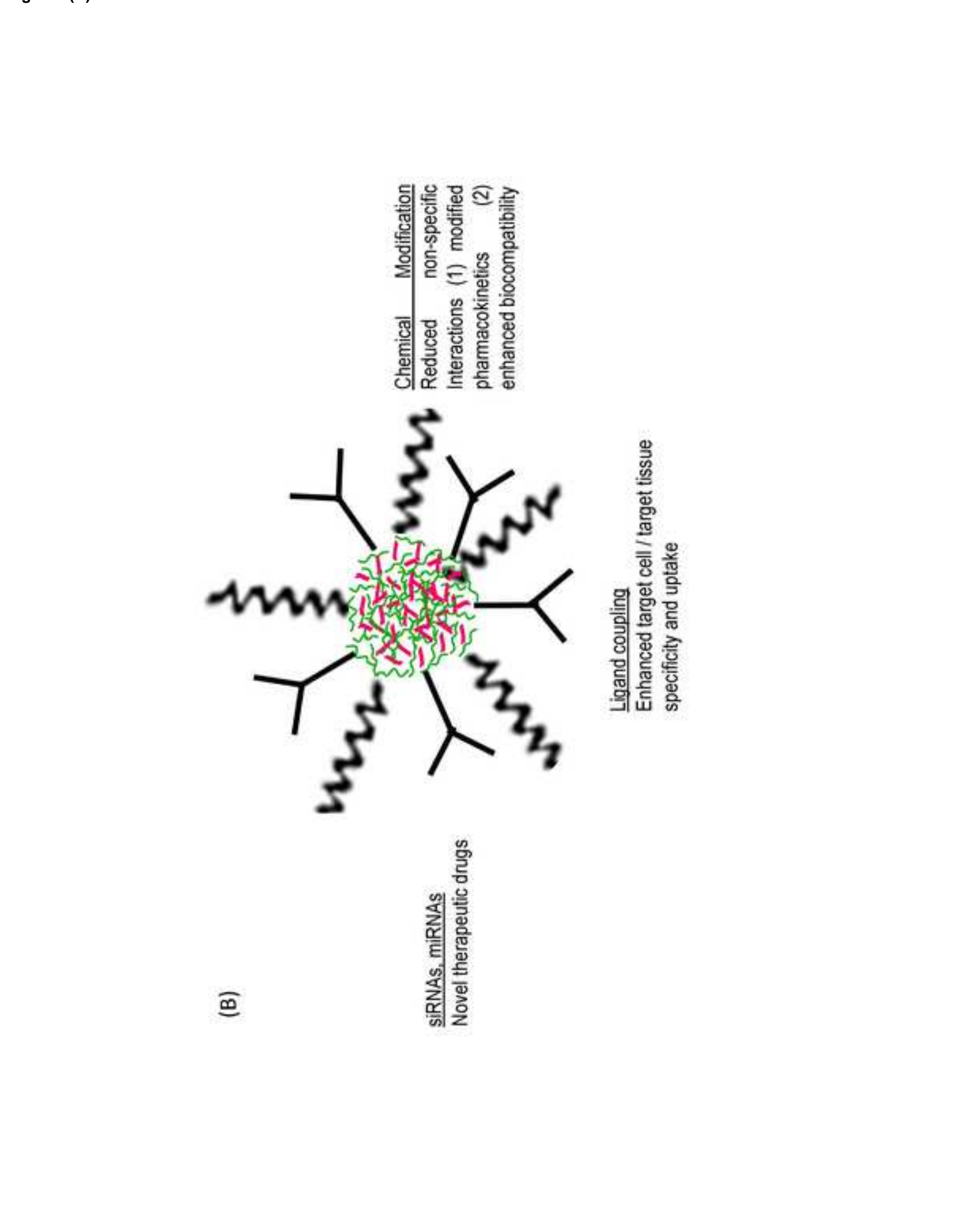
Figure $2(C)$
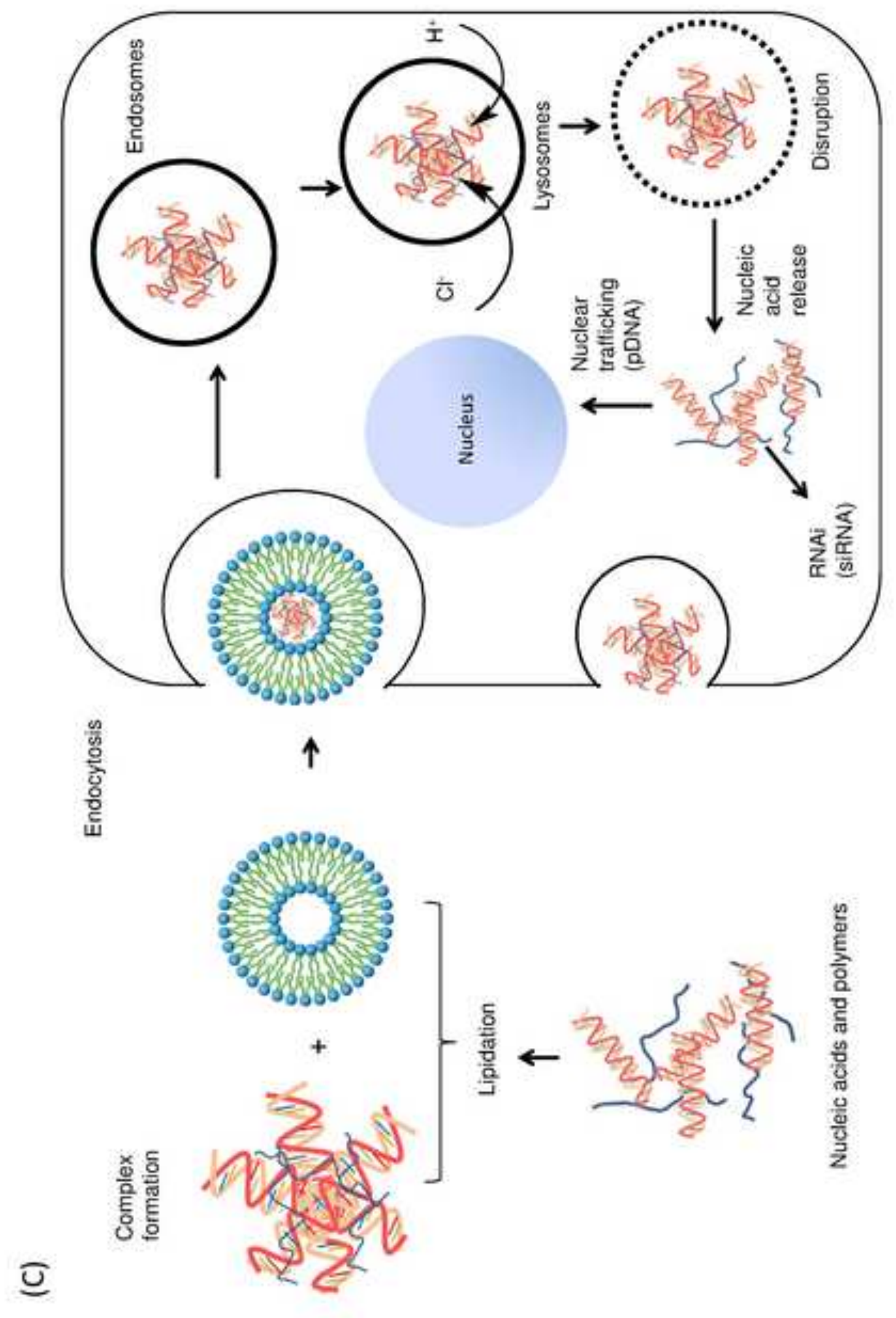

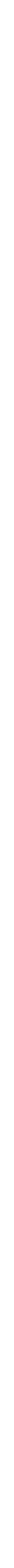

$$
\text { . }
$$
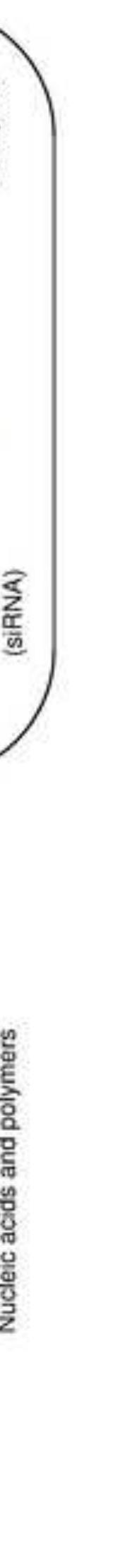

$$
\text { . }
$$

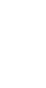
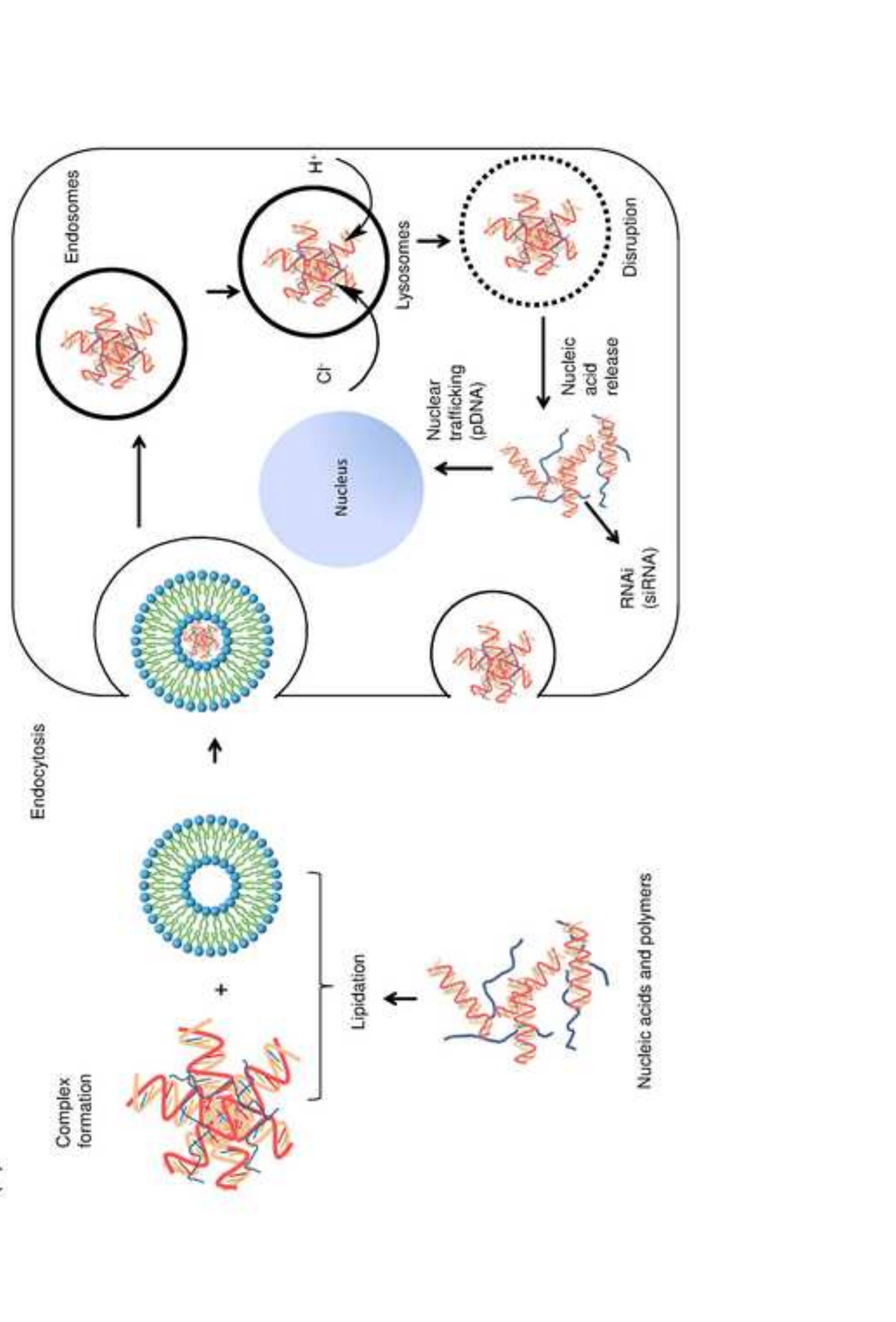

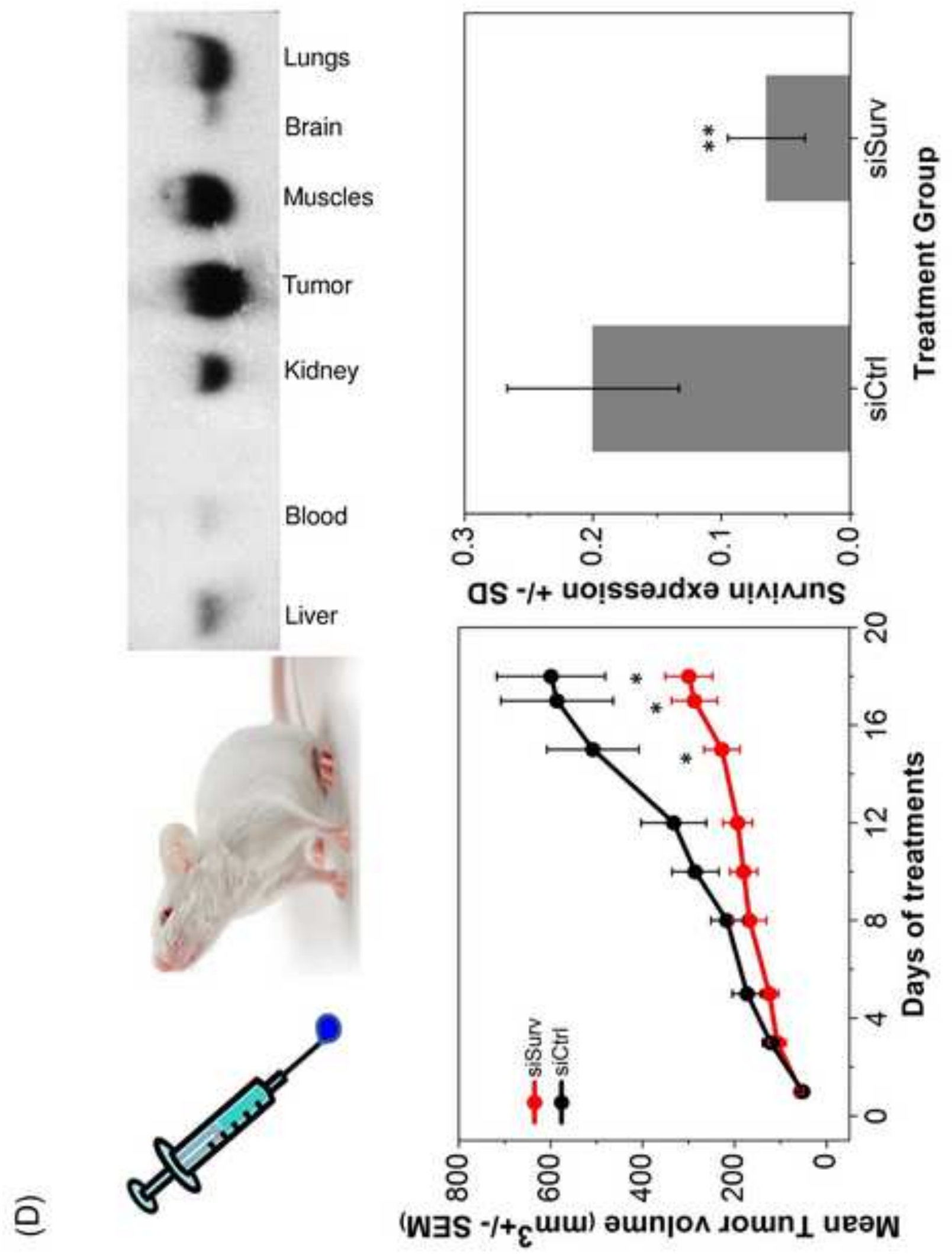

(WتS $\%+{ }_{\varepsilon}$ uuu) aunjon doun $\perp$ ueaW 
Figure 3
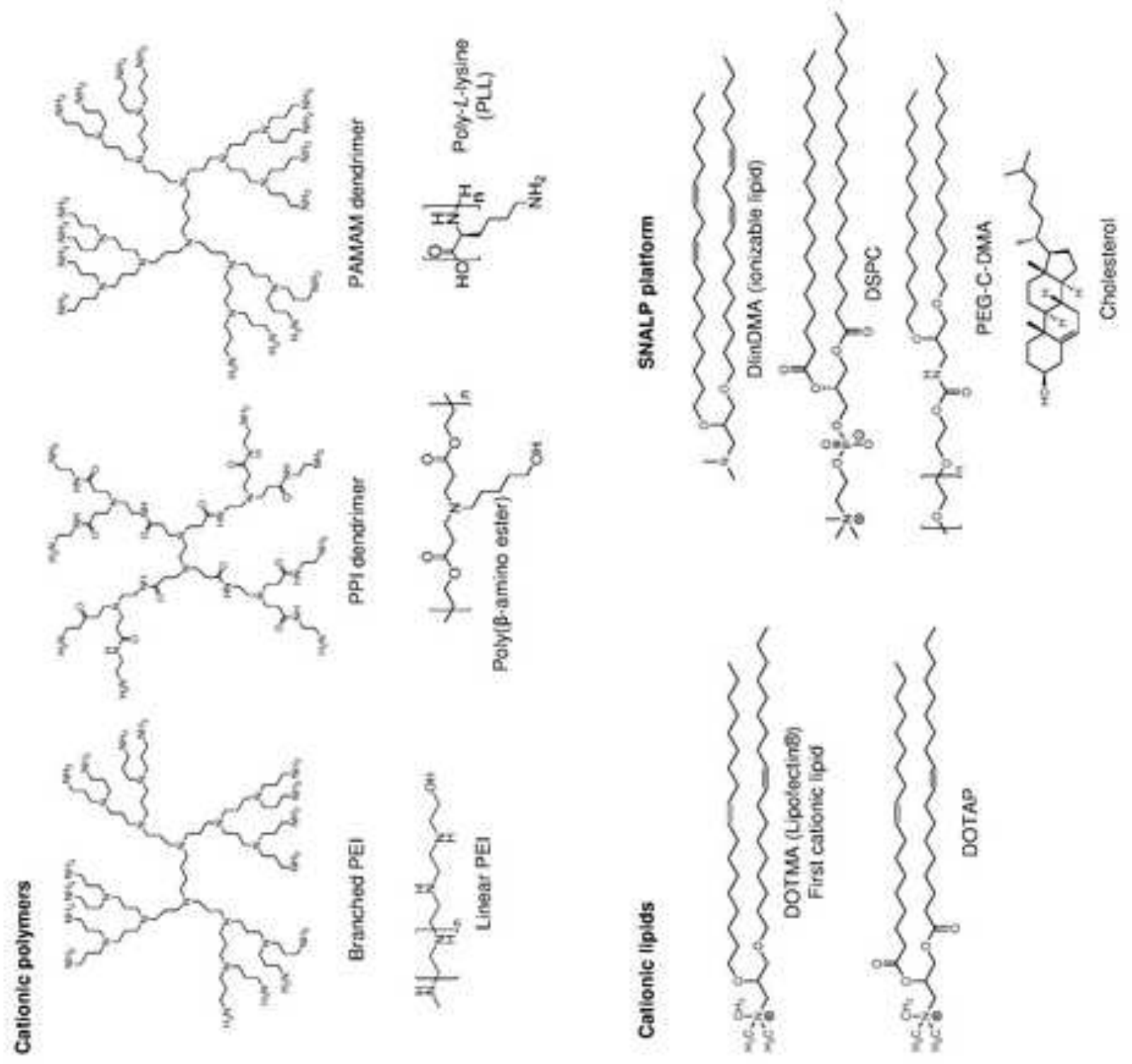


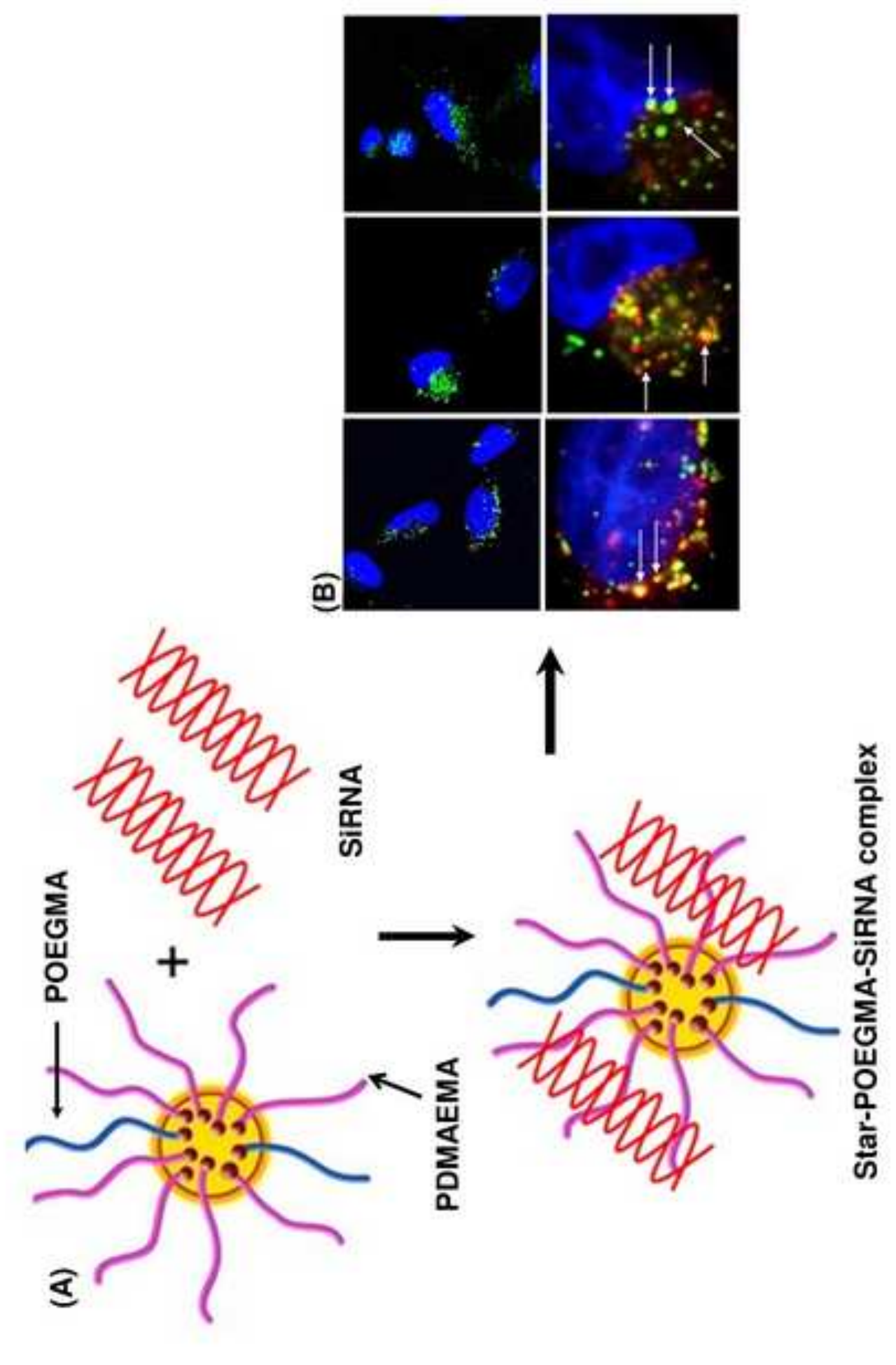

Figure 4
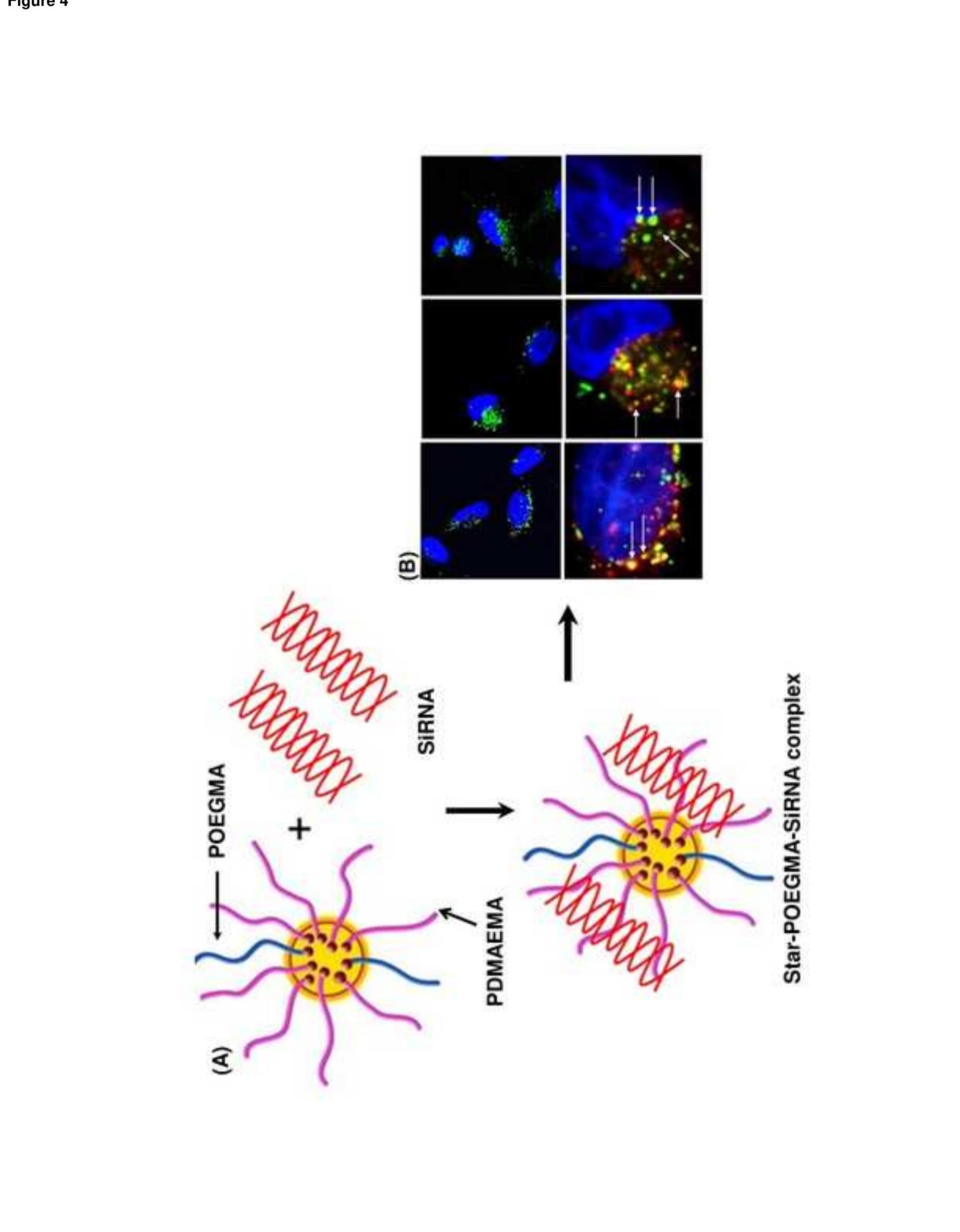

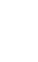

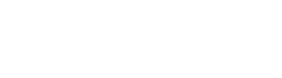




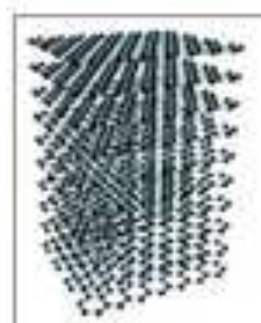

Graphite (3D)

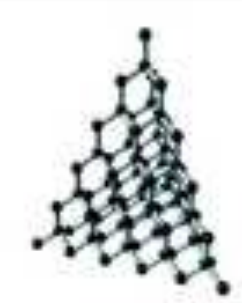

Diamond (3D)

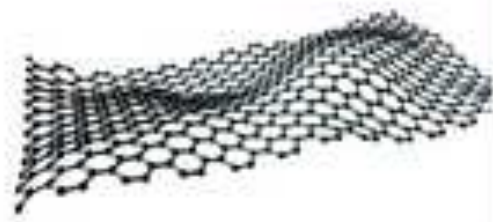

Nanoribbon (3D)

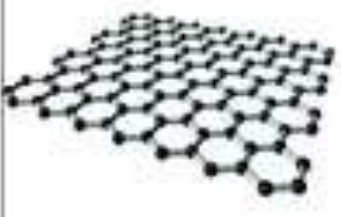

Graphene 2D

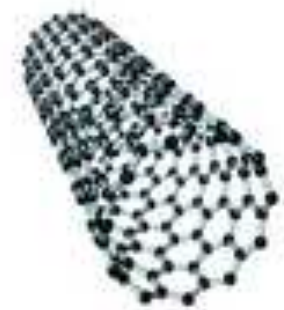

(A)

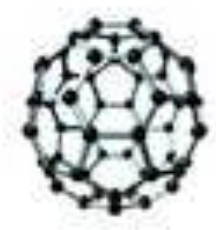

Fullerene (1D)
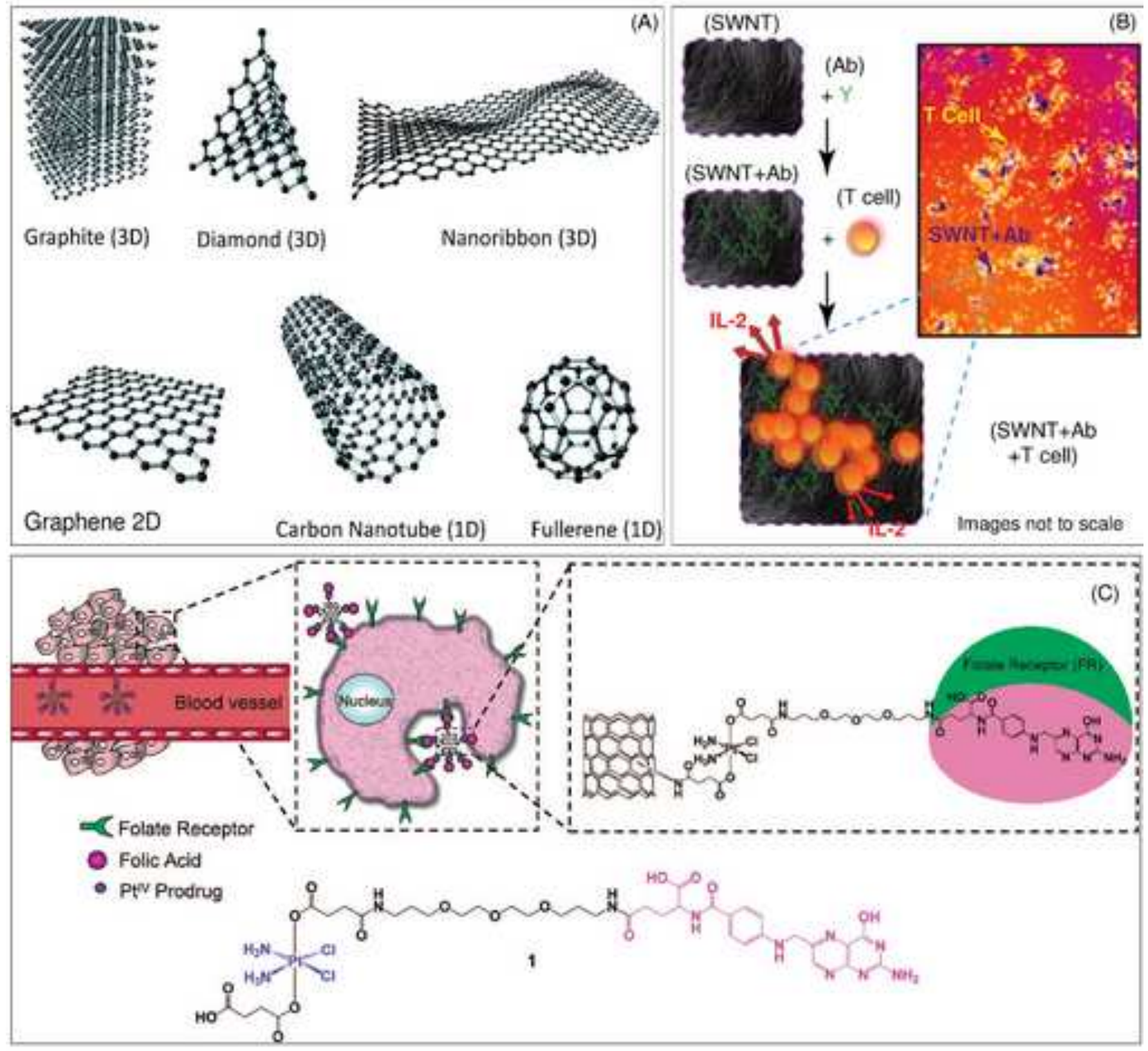


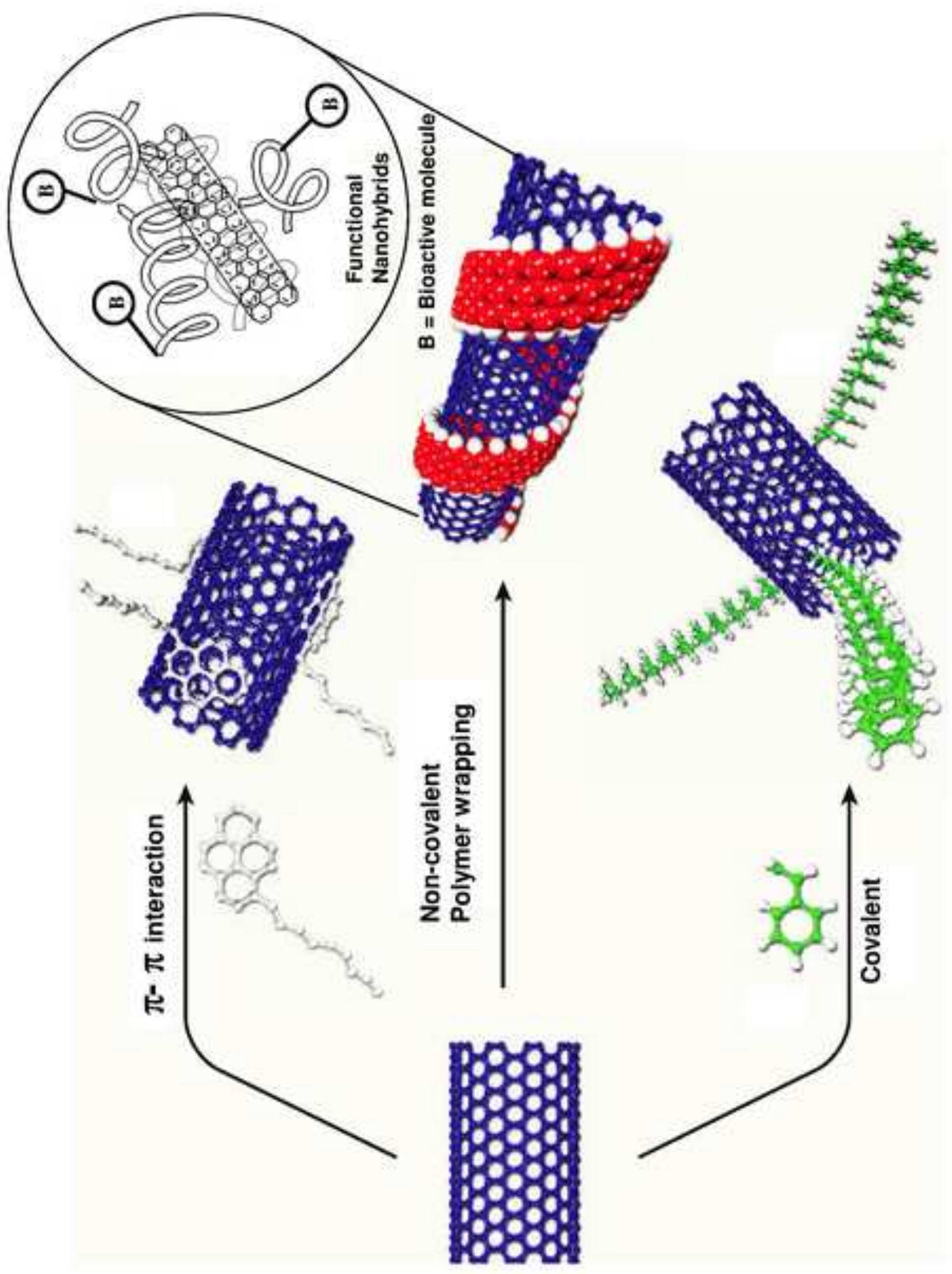




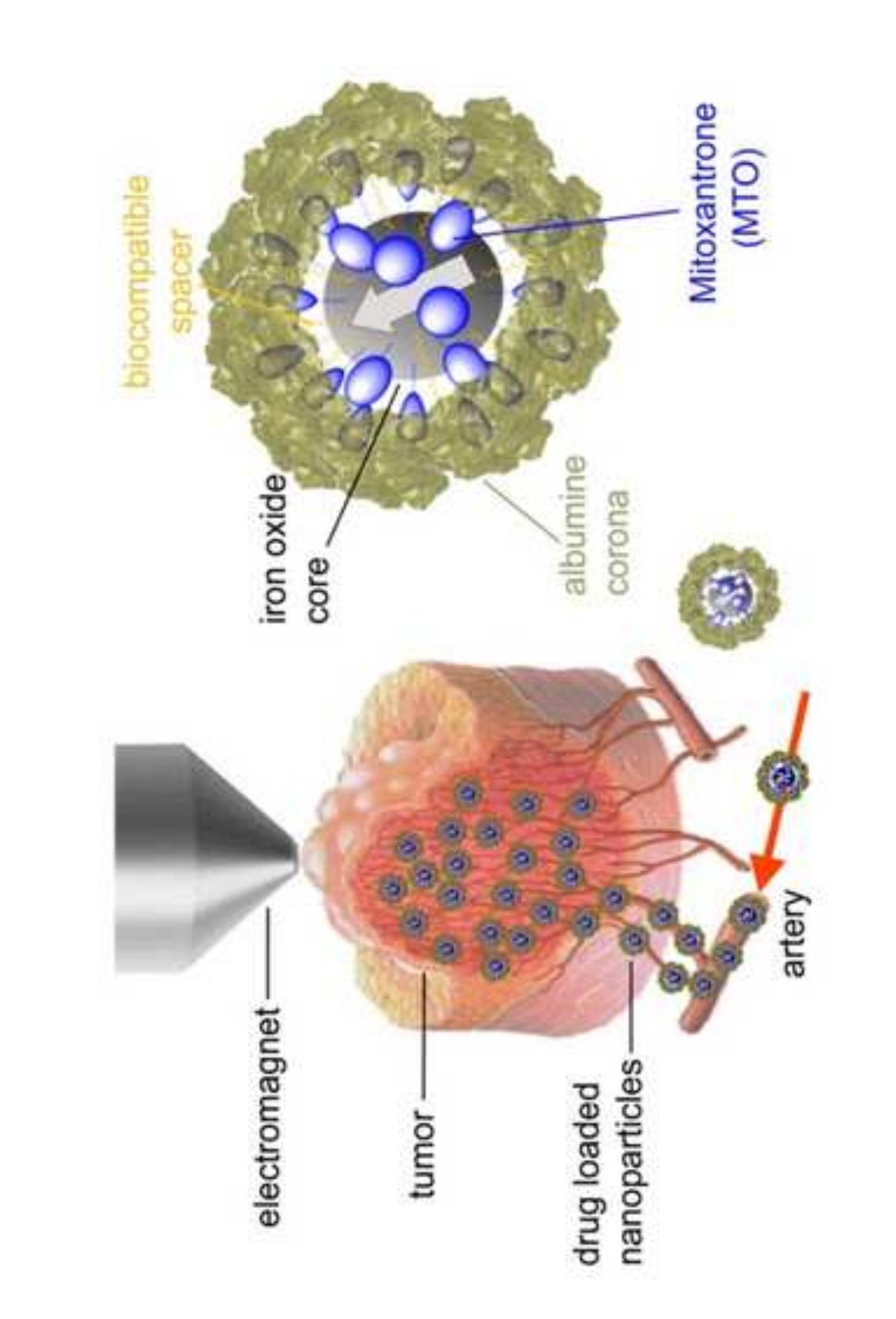

Figure 7

Fure 7
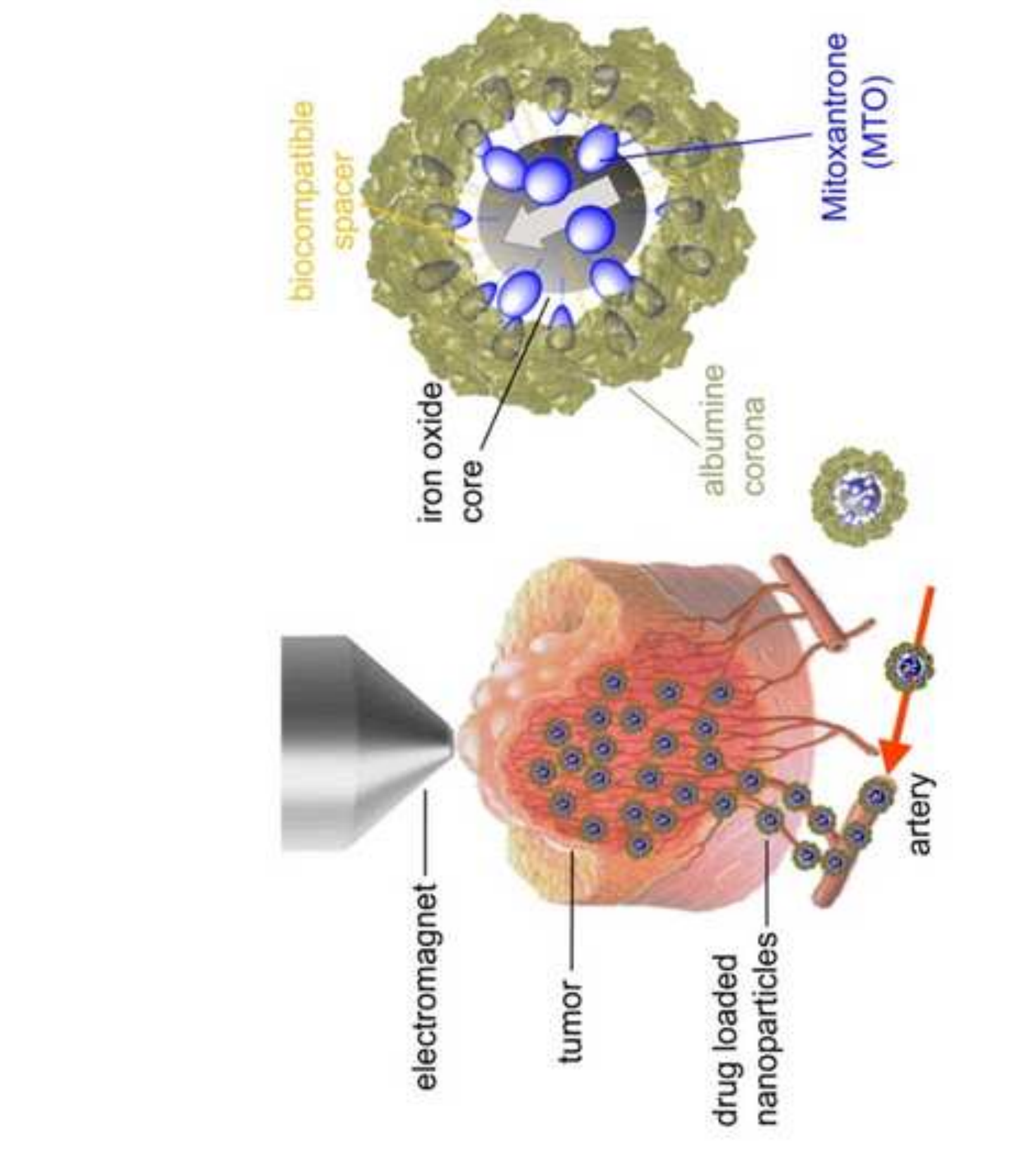

7
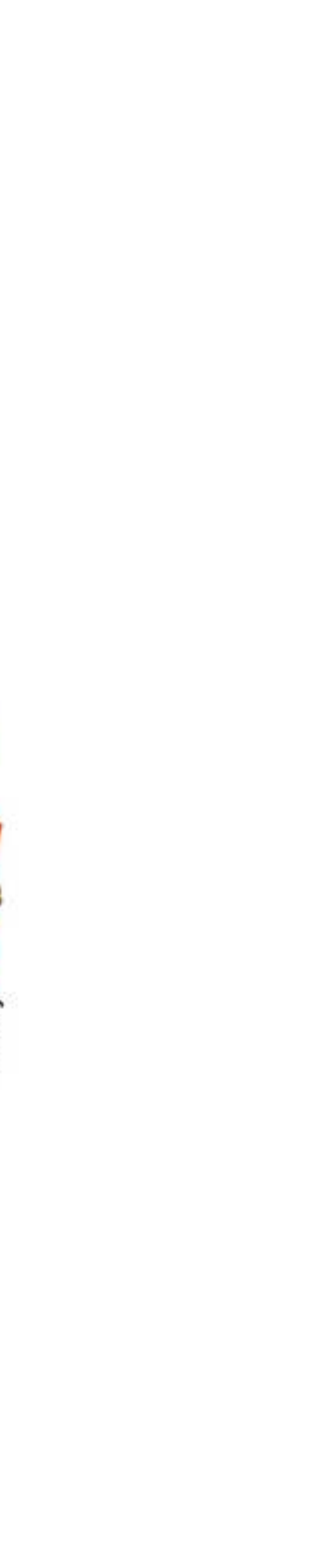
Figure 8

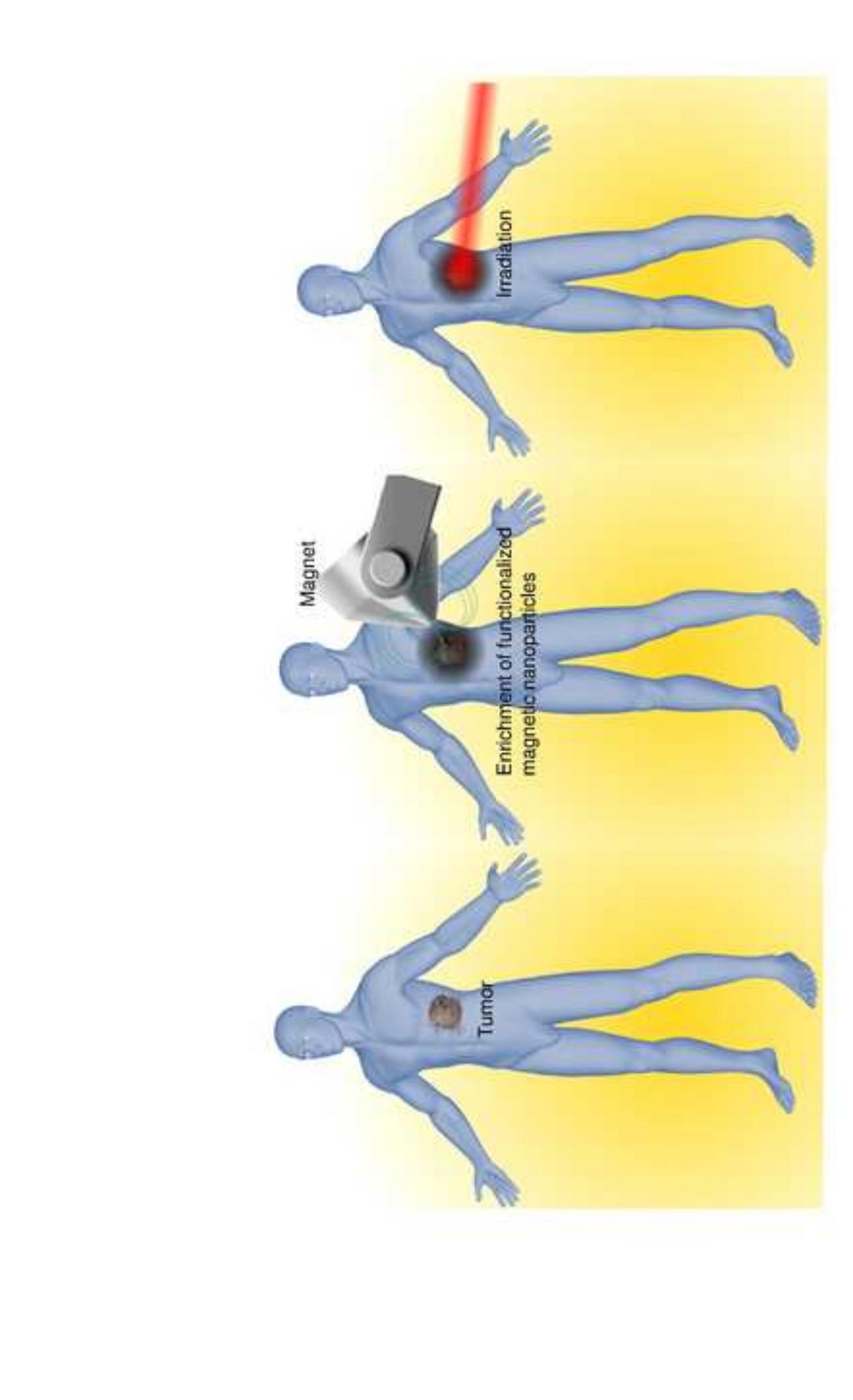

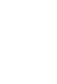

s.

8

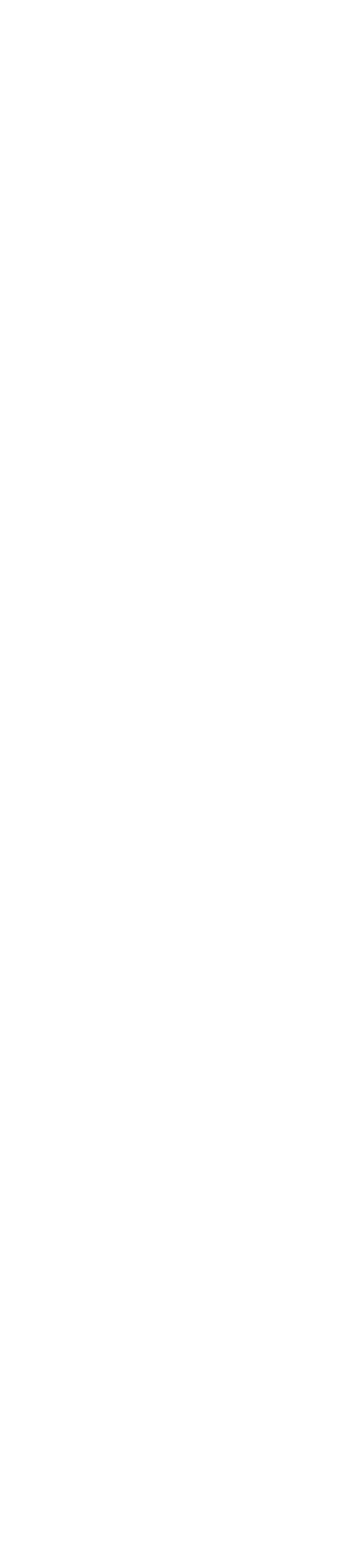




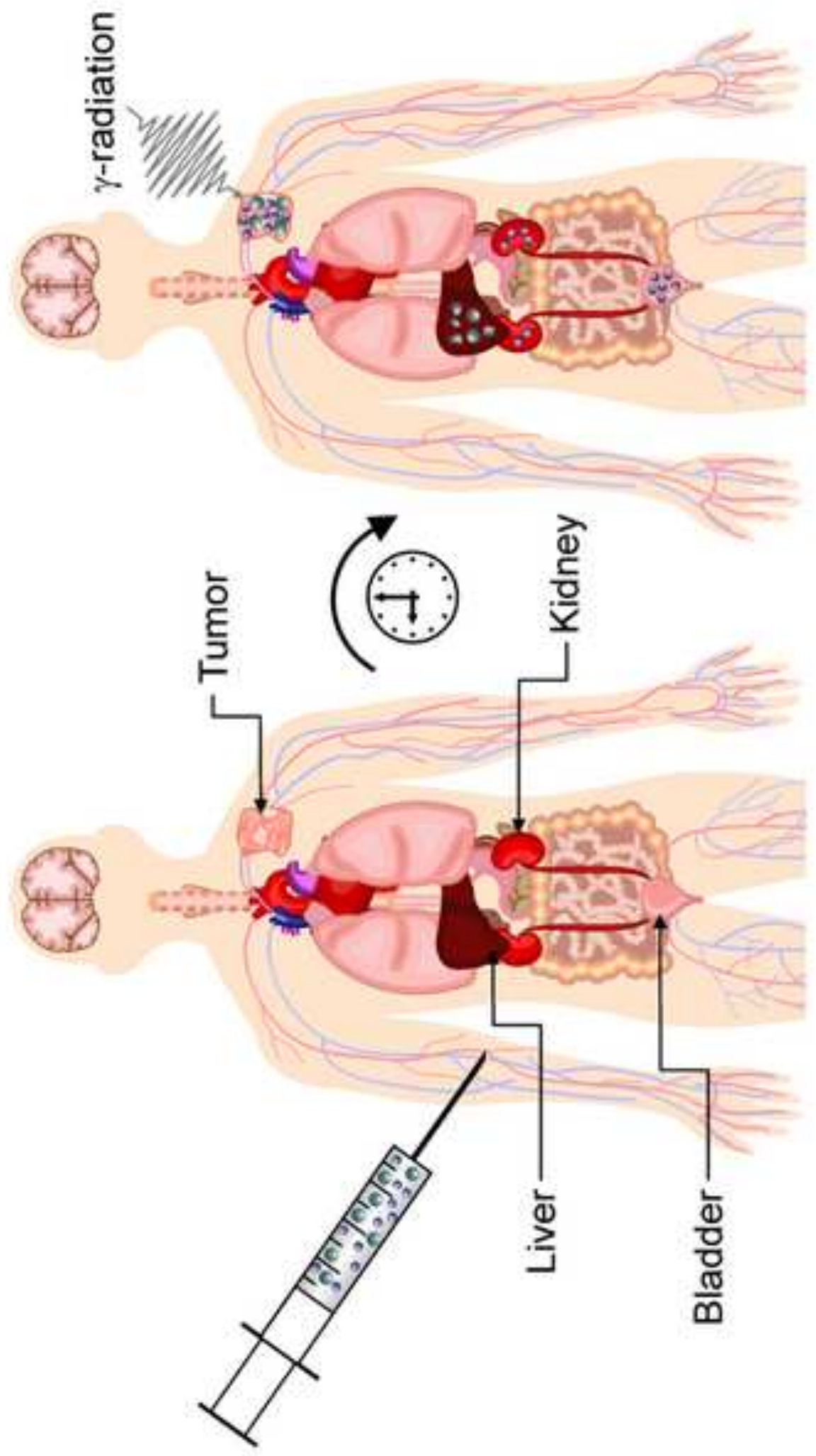




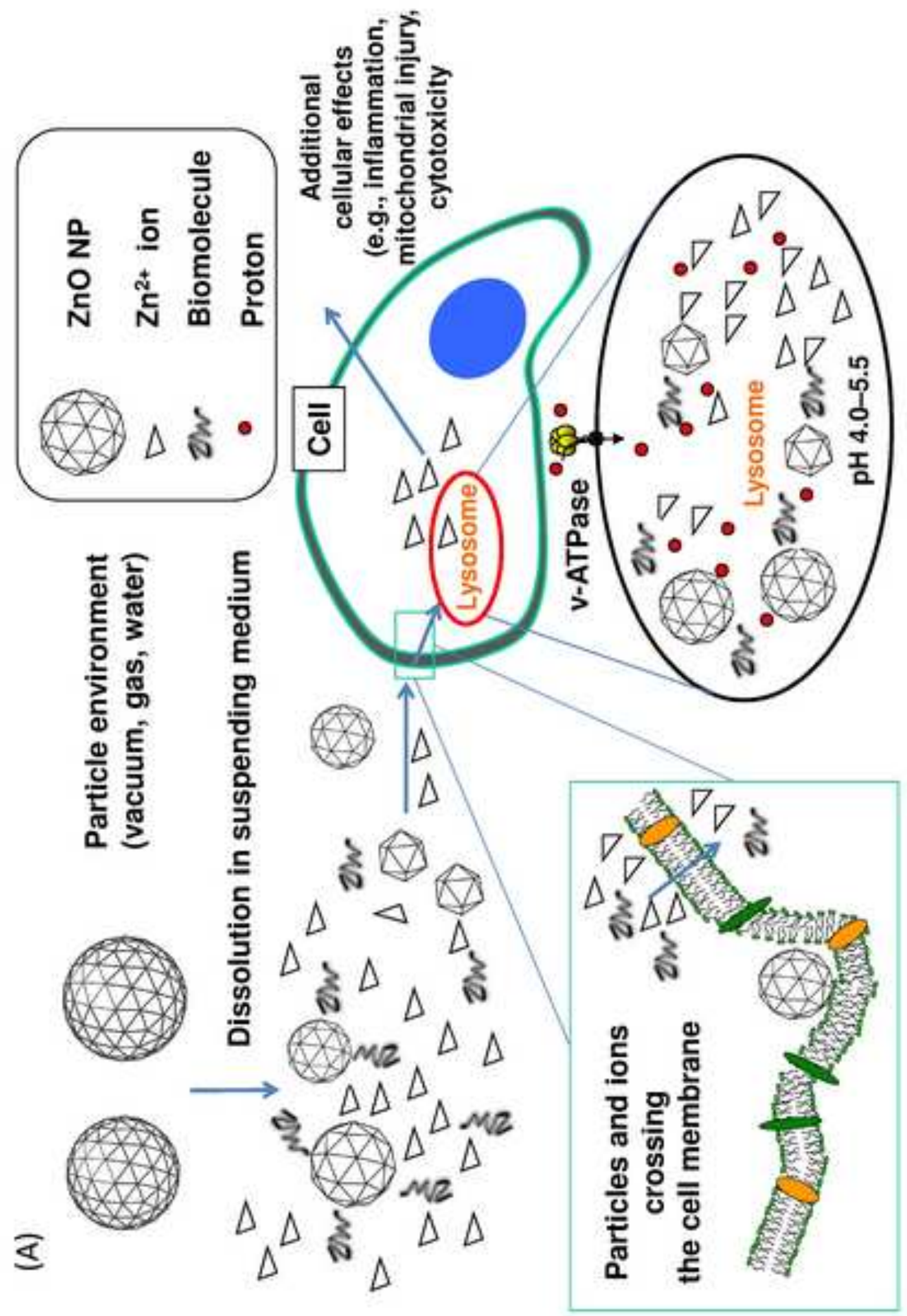


Figure $10(B)$

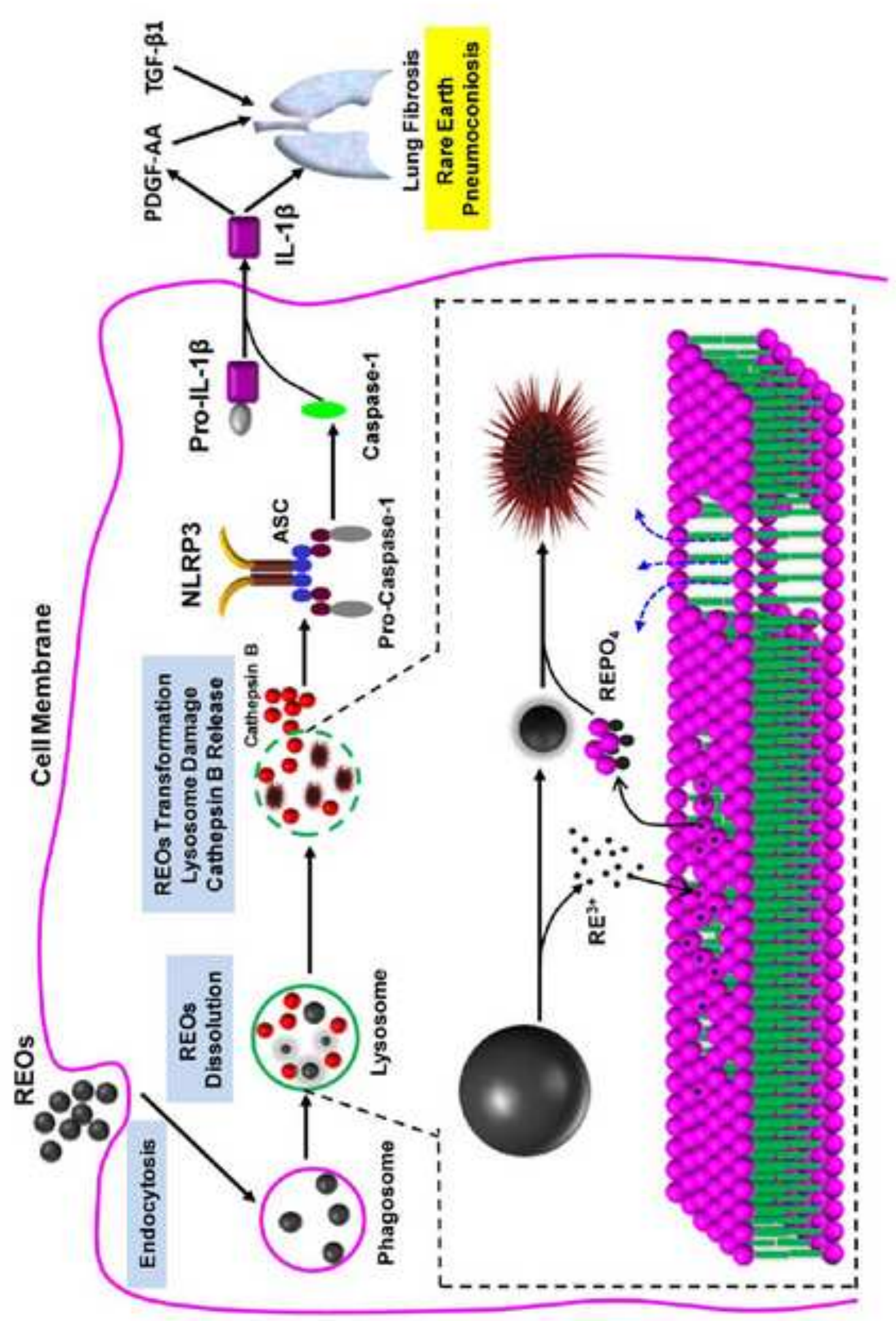



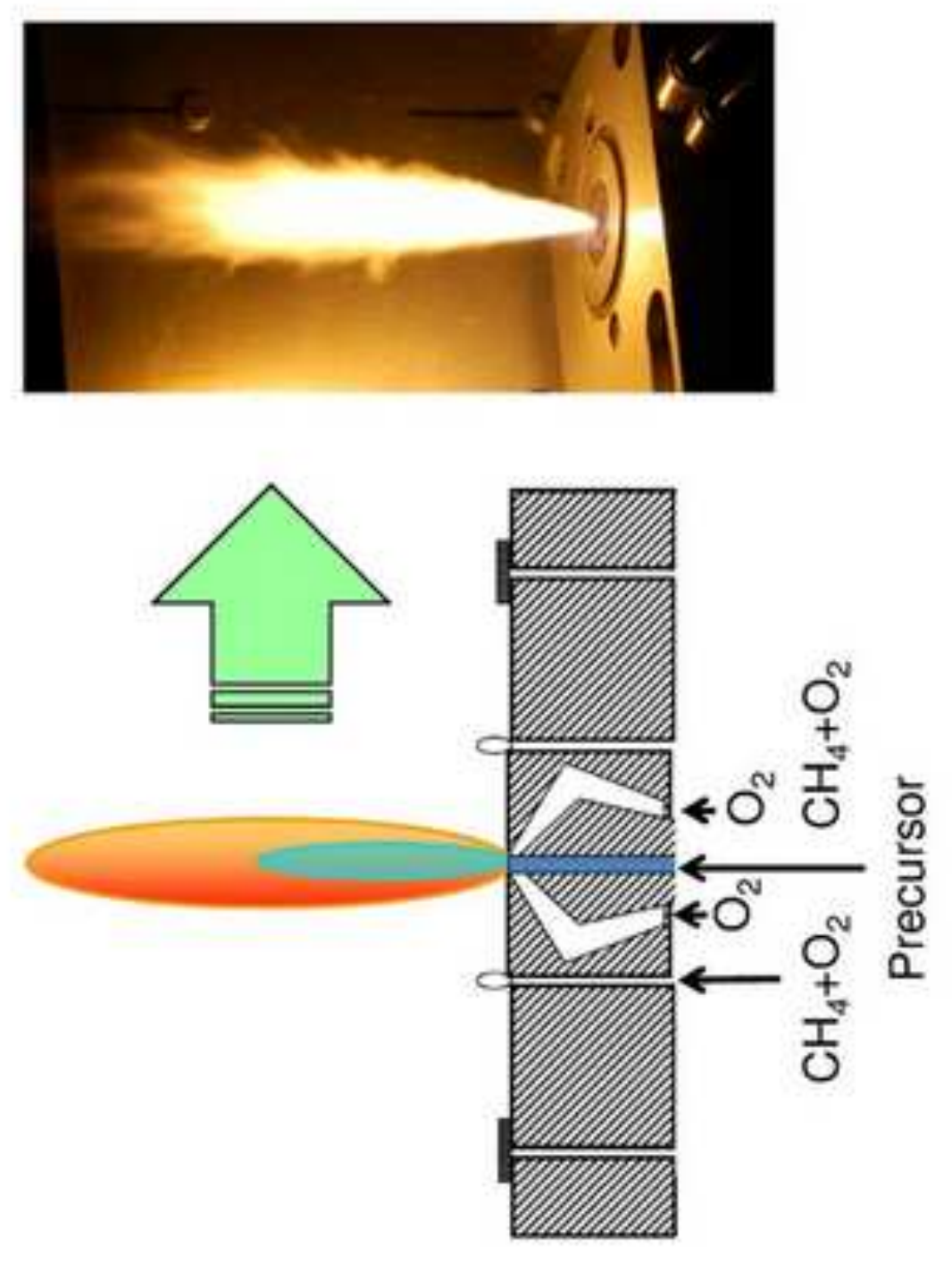

Figure 11

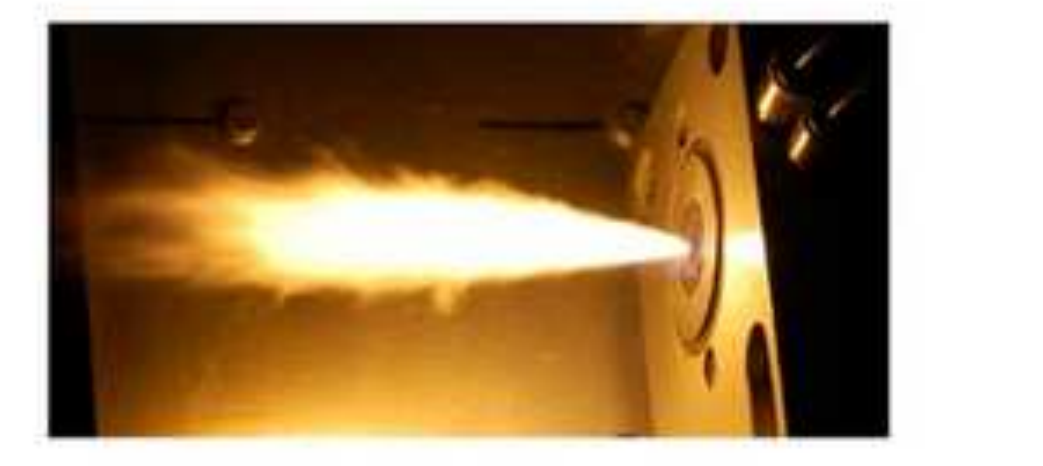



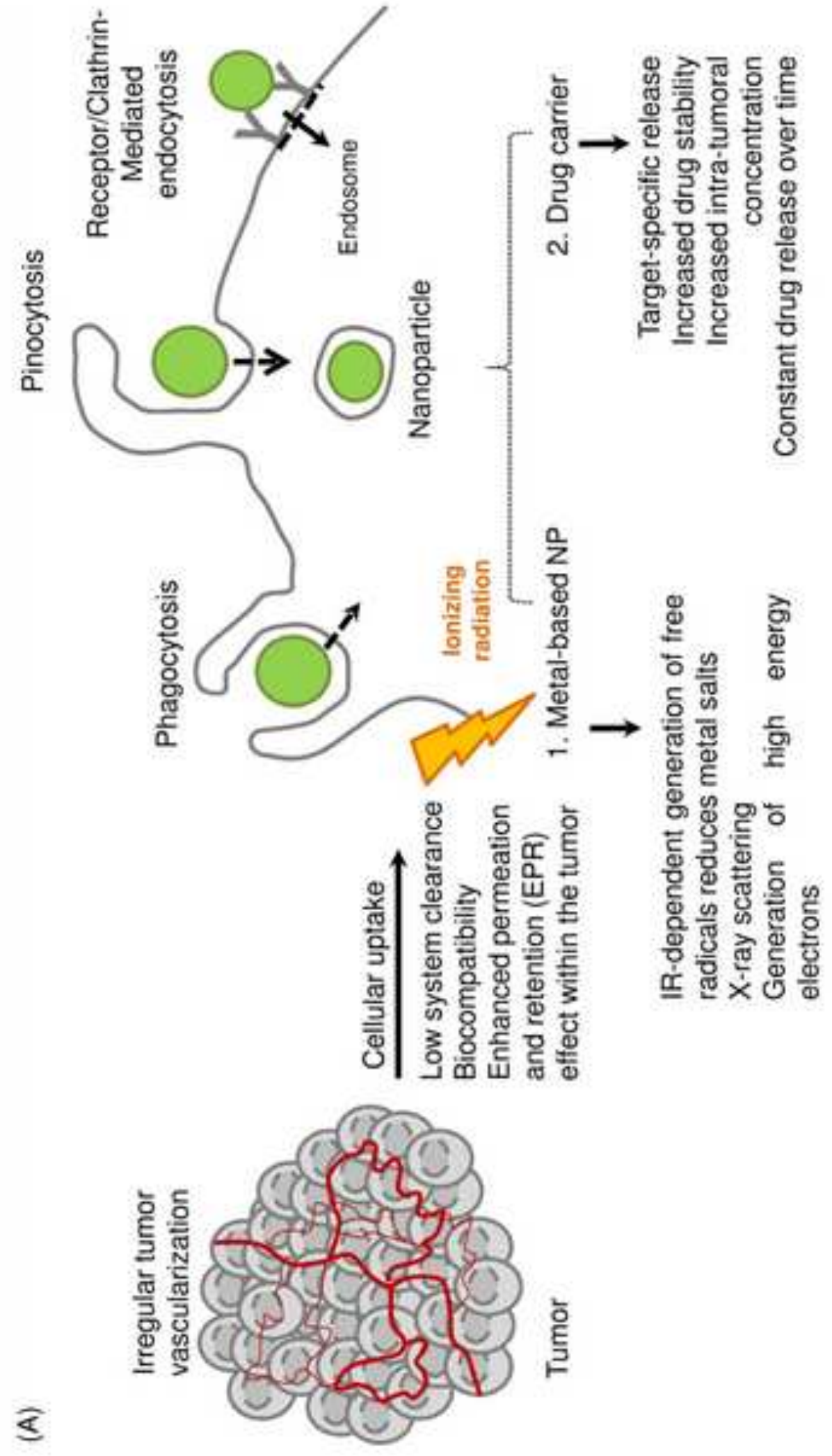

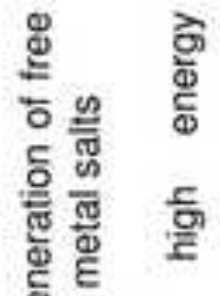

용

롤

울웡용

․ㅐ कू

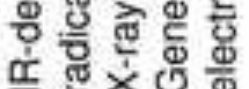

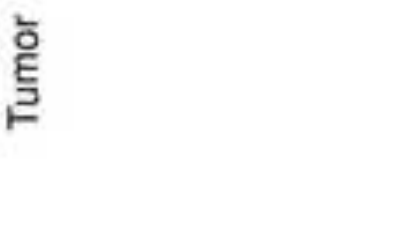




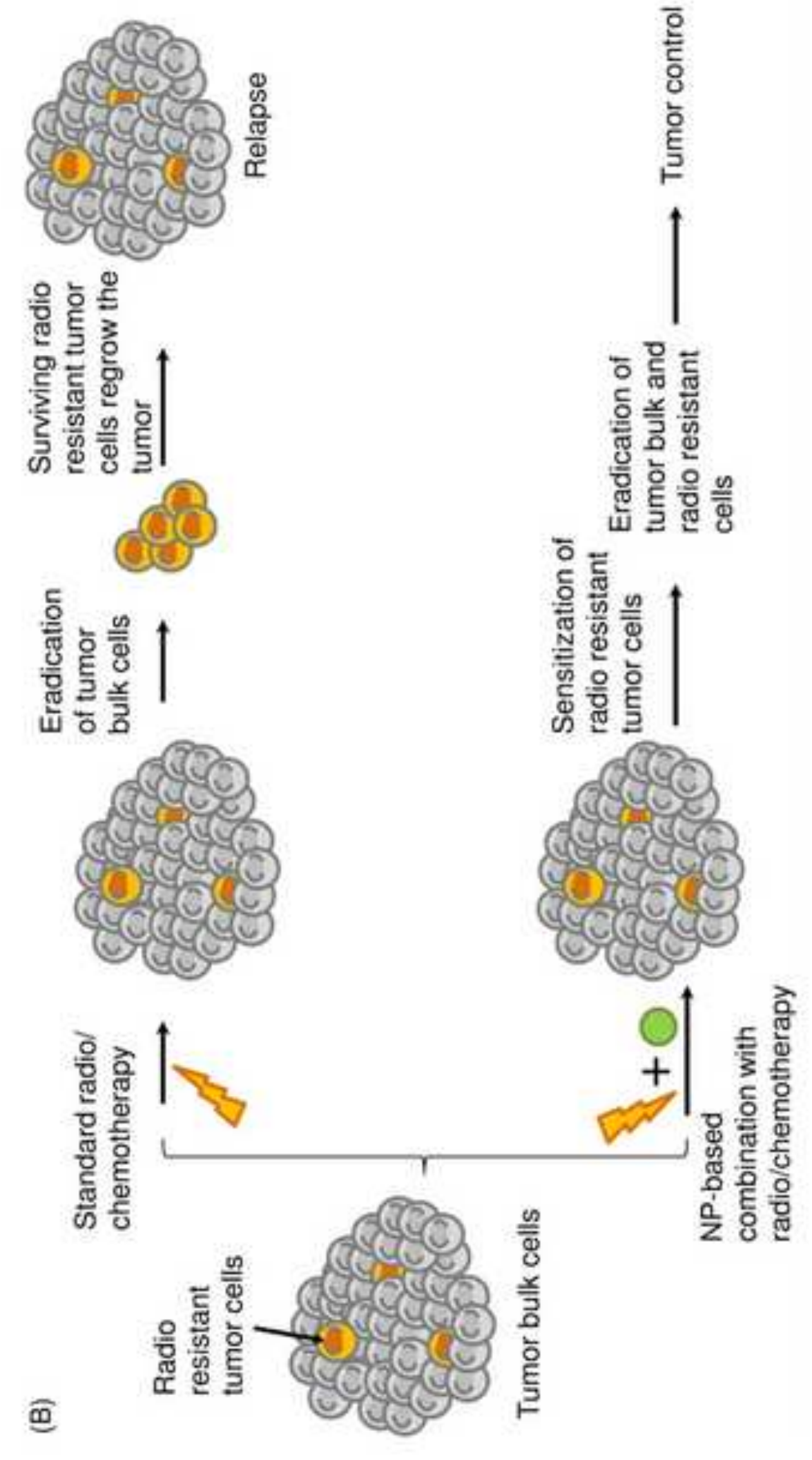

Figure 13(B) 